\title{
THE GOLDEN STANDARD TYPE Ia SUPERNOVA 2005cf: OBSERVATIONS FROM THE ULTRAVIOLET TO
} THE NEAR-INFRARED WAVEBANDS

\author{
X. Wang ${ }^{1,2}$, W. Li ${ }^{1}$, A. V. Filippenko ${ }^{1}$, R. J. Foley ${ }^{1,3,14}$, R. P. Kirshner ${ }^{3}$, M. Modjaz ${ }^{1,3,15}$, J. Bloom ${ }^{1}$, P. J. Brown ${ }^{4}$, \\ D. Carter ${ }^{5}$, A. S. Friedman ${ }^{3}$, A. Gal-Yam ${ }^{6}$, M. Ganeshalingam ${ }^{1}$, M. Hicken $^{3}$, K. Krisciunas $^{7}$, P. Milne $^{8}$, \\ J. M. Silverman ${ }^{1}$, N. B. SuntzefF ${ }^{7}$, W. M. Wood-Vasey ${ }^{3,9}$, S. B. Cenko ${ }^{1,10}$, P. Challis ${ }^{3}$, D. B. Fox ${ }^{4}$, D. Kirkman ${ }^{11}$, J. Z. Li ${ }^{2}$, \\ T. P. Li $^{2}$, M. A. Malkan ${ }^{12}$, M. R. Moore ${ }^{1}$, D. B. Reitzel ${ }^{12}$, R. M. Rich ${ }^{12}$, F. J. D. Serduke ${ }^{1}$, R. C. Shang ${ }^{2}$, T. N. Steele ${ }^{1}$, \\ B. J. SWIFT ${ }^{1}$, C. TAO ${ }^{13}$, D. S. WONG ${ }^{1}$, AND S. N. ZHANG ${ }^{2}$ \\ ${ }^{1}$ Department of Astronomy, University of California, Berkeley, CA 94720-3411, USA; wangxf@astro.berkeley.edu \\ ${ }^{2}$ Physics Department and Tsinghua Center for Astrophysics (THCA), Tsinghua University, Beijing, 100084, China; wang_xf@mail.tsinghua.edu.cn. \\ ${ }^{3}$ Harvard-Smithsonian Center for Astrophysics, 60 Garden Street, Cambridge, MA, 02138, USA \\ ${ }^{4}$ Department of Astronomy \& Astrophysics, 525 Davey Laboratory, Pennsylvania State University, University Park, PA 16802, USA \\ ${ }^{5}$ Astrophysics Research Institute, Liverpool John Moores University, Twelve Quays House, Egerton Wharf, Birkenhead CH41 1LD, UK \\ ${ }^{6}$ Benoziyo Center for Astrophysics, Weizmann Institute of Science, 76100 Rhovot, Israel \\ ${ }^{7}$ Department of Physics, Texas A\&M University, College Station, Texas, 77843, USA \\ ${ }^{8}$ Steward Observatory, University of Arizona, 933 North Cherry Avenue, Tucson, AZ 85721, USA \\ ${ }^{9}$ Department of Physics, University of Pittsburgh, 100 Allen Hall, Pittsburgh, PA 15260, USA \\ ${ }^{10}$ Space Radiation Laboratory, MS 220-47, California Institute of Technology, Pasadena, CA 91125, USA \\ ${ }^{11}$ CASS 0424, University of California, San Diego, 9500 Gilman Drive, La Jolla, CA 92093-0424, USA \\ ${ }^{12}$ Department of Physics and Astronomy, University of California, Los Angeles, CA 90095-1547, USA \\ ${ }^{13}$ Centre de Physique des Particules de Marseille, CNRS/IN2P2-Luminy, Universite de la Mediterranee, Case 907, F-13288 Marseille Cedex 9, France \\ Received 2008 November 7; accepted 2009 March 6; published 2009 May 1
}

\begin{abstract}
We present extensive photometry at ultraviolet (UV), optical, and near-infrared (NIR) wavelengths, as well as dense sampling of optical spectra, for the normal Type Ia supernova (SN Ia) 2005cf. The optical photometry, performed at eight different telescopes, shows a $1 \sigma$ scatter of $\lesssim 0.03$ mag after proper corrections for the instrument responses. From the well-sampled light curves, we find that SN $2005 \mathrm{cf}$ reached a $B$-band maximum at $13.63 \pm 0.02 \mathrm{mag}$, with an observed luminosity decline rate $\Delta m_{15}(B)=1.05 \pm 0.03 \mathrm{mag}$. The correlations between the decline rate and various color indexes, recalibrated on the basis of an expanded SN Ia sample, yield a consistent estimate for the host-galaxy reddening of SN $2005 \mathrm{cf}, E(B-V)_{\text {host }}=0.10 \pm 0.03 \mathrm{mag}$. The UV photometry was obtained with the Hubble Space Telescope and the Swift Ultraviolet/Optical Telescope, and the results match each other to within $0.1-0.2$ mag. The UV light curves show similar evolution to the broadband $U$, with an exception in the 2000-2500 $\AA$ spectral range (corresponding to the F220W/uvm 2 filters), where the light curve appears broader and much fainter than that on either side (likely owing to the intrinsic spectral evolution). Combining the UV data with the ground-based optical and NIR data, we establish the generic UVoptical-NIR bolometric light curve for SN 2005cf and derive the bolometric corrections in the absence of UV and/or NIR data. The overall spectral evolution of SN $2005 \mathrm{cf}$ is similar to that of a normal SN Ia, but with variety in the strength and profile of the main feature lines. The spectra at early times displayed strong, high-velocity (HV) features in the Ca II H\&K doublet and NIR triplet, which were distinctly detached from the photosphere $\left(v \approx 10,000 \mathrm{~km} \mathrm{~s}^{-1}\right)$ at a velocity ranging from 20,000 to $25,000 \mathrm{~km} \mathrm{~s}^{-1}$. One interesting feature is the flatbottomed absorption observed near $6000 \AA$ in the earliest spectrum, which rapidly evolved into a triangular shape and then became a normal Si II $\lambda 6355$ absorption profile at about one week before maximum brightness. This premaximum spectral evolution is perhaps due to the blending of the Si II $\lambda 6355$ at photospheric velocity and another HV absorption component (e.g., an Si II shell at a velocity $\sim 18,000 \mathrm{~km} \mathrm{~s}^{-1}$ ) in the outer ejecta, and may be common in other normal SNe Ia. The possible origin of the HV absorption features is briefly discussed.
\end{abstract}

Key words: supernovae: general - supernovae: individual (SN 2005cf)

Online-only material: color figures

\section{INTRODUCTION}

Type Ia supernovae (SNe Ia) play important roles in diverse areas of astrophysics, from the chemical evolution of galaxies to observational cosmology. They, together with the core-collapse $\mathrm{SNe}$, are responsible for most of the heavy elements in the universe. SNe Ia have also been used over the past decade as the most powerful tool probing the expansion history of the universe. Owing to a relatively homogeneous origin—-probably

\footnotetext{
${ }^{14}$ Clay Fellow.

${ }^{15}$ Miller Fellow.
}

an accreting carbon-oxygen white dwarf (WD) with a mass close to the Chandrasekhar limit $\left(\sim 1.4 M_{\odot}\right)$ in a binary system (for a review, see Hillebrandt \& Niemeyer 2000)—most SNe Ia show strikingly similar spectral and photometric behavior (e.g., Branch \& Tammann 1992; Suntzeff 1996; Filippenko 1997). In particular, the observed peak luminosities of SNe Ia have been shown to correlate with the shapes of their light or color curves (e.g., Phillips 1993; Hamuy et al. 1996a, 1996b; Riess et al. 1996; Perlmutter et al. 1997; Wang et al. 2003a, 2003b; Wang et al. 2005; Guy et al. 2005; Prieto et al. 2006; Jha et al. 2007; Guy et al. 2007; Conley et al. 2008), leading to an uncertainty of $\lesssim 10 \%$ in distance measurements from SNe Ia. 
Based on observations of SNe Ia at redshifts $z \approx 0.4-0.5$, Riess et al. (1998) and Perlmutter et al. (1999) first reported the discovery of the accelerating expansion of the universe. The evidence for acceleration from SNe Ia improved markedly with follow-up studies (Tonry et al. 2003; Knop et al. 2003; Barris et al. 2004; Riess et al. 2004, 2007; Astier et al. 2006; Wood-Vasey et al. 2007), suggesting that $\sim 70 \%$ of the universe is composed of a mysterious dark energy (for a review, see, e.g., Filippenko 2005a). Elucidating the nature of dark energy requires a large sample of well-observed $\mathrm{SNe}$ Ia at even higher redshifts $(z \gtrsim 0.7)$, and also relies on the improvement of the SN Ia standardization (e.g., $\lesssim 0.01 \mathrm{mag}$ ). Progress can be made by searching for additional luminosity-dependent parameters, or by identifying a subsample of SNe Ia with the lowest scatter in luminosity (X. Wang et al. 2009, in preparation; Branch et al. 2009). This depends on the degree of our understanding of SN Ia physics as well as on controlling various systematic effects such as the photometry itself, SN luminosity evolution, and absorption by dust. Clarification of the above issues demands a large sample of SNe Ia with well observed spectra and light curves, from which we can get better constraints on their physical properties.

Quite a few detailed studies have been conducted of spectroscopically and/or photometrically peculiar SNe Ia such as SNe 1991 T (Filippenko et al. 1992a; Phillips et al. 1992), 1991bg (Filippenko et al. 1992b, Leibundgut et al. 1993), 2000cx (Li et al. 2001; Thomas et al. 2004; Candia et al. 2003), 2002cx (Li et al. 2003; Branch et al. 2004; Jha et al. 2006a), and 2006gz (Hicken et al. 2007). A comparable number of relatively normal SNe Ia have also been individually studied, including SNe 1994D (Patat et al. 1996), 1996X (Salvo et al. 2001), 1998aq (Branch et al. 2003; Riess et al. 2005), 1998bu (Jha et al. 1999), 1999ee (Stritzinger et al. 2002; Hamuy et al. 2002), 2001el (Krisciunas et al. 2003), 2002er (Pignata et al. 2004; Kotak et al. 2005), 2003cg (Elias-Rosa et al. 2006), 2003du (Stanishev et al. 2007), 2004eo (Pastorello et al. 2007a), 2002bo (Benetti et al. 2004), and 2006X (Wang et al. 2008a), though the last two may differ from other typical SNe Ia given the unusually high expansion velocity of their photospheres and a relatively flat evolution of their $B$-band light curves starting from the early nebular phase (Wang et al. 2008a). However, a much larger sample of SNe Ia must be investigated in order to determine the dispersion among their properties and refine possible systematic effects for precision cosmology. In addition, the sample of "golden standard" SNe Ia having extensive observations from ultraviolet (UV) through near-infrared (NIR) wavelengths is sparse. The UV properties could provide clues to the diversity and evolution of the progenitor systems, as they are sensitive to the metallicity of the ejecta as well as the degree of mixing of the synthesized ${ }^{56} \mathrm{Ni}$ (Höflich et al. 1998; Blinnikov \& Sorokina 2000), while the NIR data are particularly suitable for the study of dust properties and the determination of absorption corrections. The UV and NIR data are also important in helping to determine, by means of the light curves, the bolometric luminosity of SNe Ia.

In this paper, we present extensive observations of the SN Ia 2005cf in UV, optical, and NIR bands, providing a "golden standard" with which to compare other SNe Ia. Pastorello et al. (2007b; hereafter P07) and Garavini et al. (2007; hereafter G07) have previously studied the optical properties of SN 2005cf, but our unique UV data along with an excellent independent optical/NIR data set allow us to provide better constraints on the properties of SN 2005cf. We compare our results with those of P07 and G07 where appropriate. Our observations and data reduction are described in Section 2, while Section 3 presents the UV-optical-NIR (uvoir) light curves, the color curves, and the reddening estimate. The spectral evolution is presented in Section 4. In Section 5 we construct the bolometric light curve of SN 2005cf. Our discussion and conclusions are given in Section 6 .

\section{OBSERVATIONS AND DATA REDUCTION}

SN 2005cf was discovered at an unfiltered magnitude of 16.4 on 2005 May 28.36 (UT dates are used throughout this paper) by Pugh \& Li (2005) during the Lick Observatory Supernova Search (LOSS) with the $0.76 \mathrm{~m}$ Katzman Automatic Imaging Telescope (KAIT; Filippenko et al. 2001; Filippenko 2005b), with $\mathrm{J} 2000$ coordinates $\alpha=15^{\mathrm{h}} 21^{\mathrm{m}} 32^{\mathrm{s}} .21$ and $\delta=-07^{\circ} 24^{\prime} 47^{\prime \prime} .5$. It exploded in the vicinity of the tidal bridge connecting the S0 galaxy MCG-01-39-003 with the nearby Sb galaxy MCG-0139-002 (NGC 5917); see Figure 1. Assuming MCG-01-39-003 is the host galaxy of SN 2005cf, we find that the SN was 15".7 west and 123".0 north of the galaxy nucleus.

An optical spectrum taken on 2006 May 31.22 revealed that SN 2005 cf was a very young SN Ia, at a phase of $\sim 10$ days before maximum brightness (Modjaz et al. 2005). On this basis, we requested frequent optical and NIR imaging, as well as optical spectroscopy; we collected a total of 634 photometric data points and 40 optical spectra. Moreover, Hubble Space Telescope (HST) UV and NIR observations were soon triggered (proposal GO-10182; PI Filippenko) with the Advanced Camera for Surveys (ACS) and the Near-Infrared Camera and Multi-Object Spectrometer (NICMOS) at 12 different epochs. UV and optical photometry was also obtained with the Ultraviolet/Optical Telescope (UVOT) on the space-based Swift telescope.

\subsection{Ground-Based Observations}

\subsubsection{Optical and NIR Photometry}

The ground-based optical photometry of SN 2005cf, spanning from 12 days before to three months after the $B$-band maximum, was obtained with the following telescopes: (1) the KAIT and the $1.0 \mathrm{~m}$ Nickel telescope at Lick Observatory in California; (2) the $1.2 \mathrm{~m}$ telescope at the Fred Lawrence Whipple Observatory (FLWO) of the Harvard-Smithsonian Center for Astrophysics (CfA) in Arizona; (3) the $1.3 \mathrm{~m}$ and $0.9 \mathrm{~m}$ telescopes at Cerro Tololo Inter-American Observatory (CTIO) in Chile; (4) the 1.5 $\mathrm{m}$ telescope at Palomar Observatory in California (Cenko et al. 2006); (5) the $2.0 \mathrm{~m}$ Liverpool telescope at La Palma; and (6) the $0.8 \mathrm{~m}$ THCA-NAOC Telescope (TNT) at Beijing Xinglong Observatory (BAO) in China. Broadband $B V R I$ photometry was obtained with all of the above telescopes, except for the FLWO $1.2 \mathrm{~m}$ and Liverpool $2.0 \mathrm{~m}$ telescopes which followed SN 2005cf in $B V$ and Sloan $r i$ filters. Observations made with the KAIT, the FLWO $1.2 \mathrm{~m}$, the CTIO $0.9 \mathrm{~m}$, and the Nickel $1.0 \mathrm{~m}$ also sampled the $U$ band. The NIR $\left(J H K_{s}\right)$ photometry was obtained with the $1.3 \mathrm{~m}$ Peters Automated Infrared Imaging Telescope (PAIRITEL; Bloom et al. 2006) at the FLWO.

As part of routine processing, all CCD images were corrected for bias, flatfielded, and cleaned of cosmic rays. Since SN $2005 \mathrm{cf}$ is isolated far away from the host-galaxy center, we omitted the usual step of subtracting the galaxy template from the SN images; instead, the foreground sky was determined locally and subtracted. Instrumental magnitudes of the SN and the local standard stars (labeled in Figure 1) were measured by 


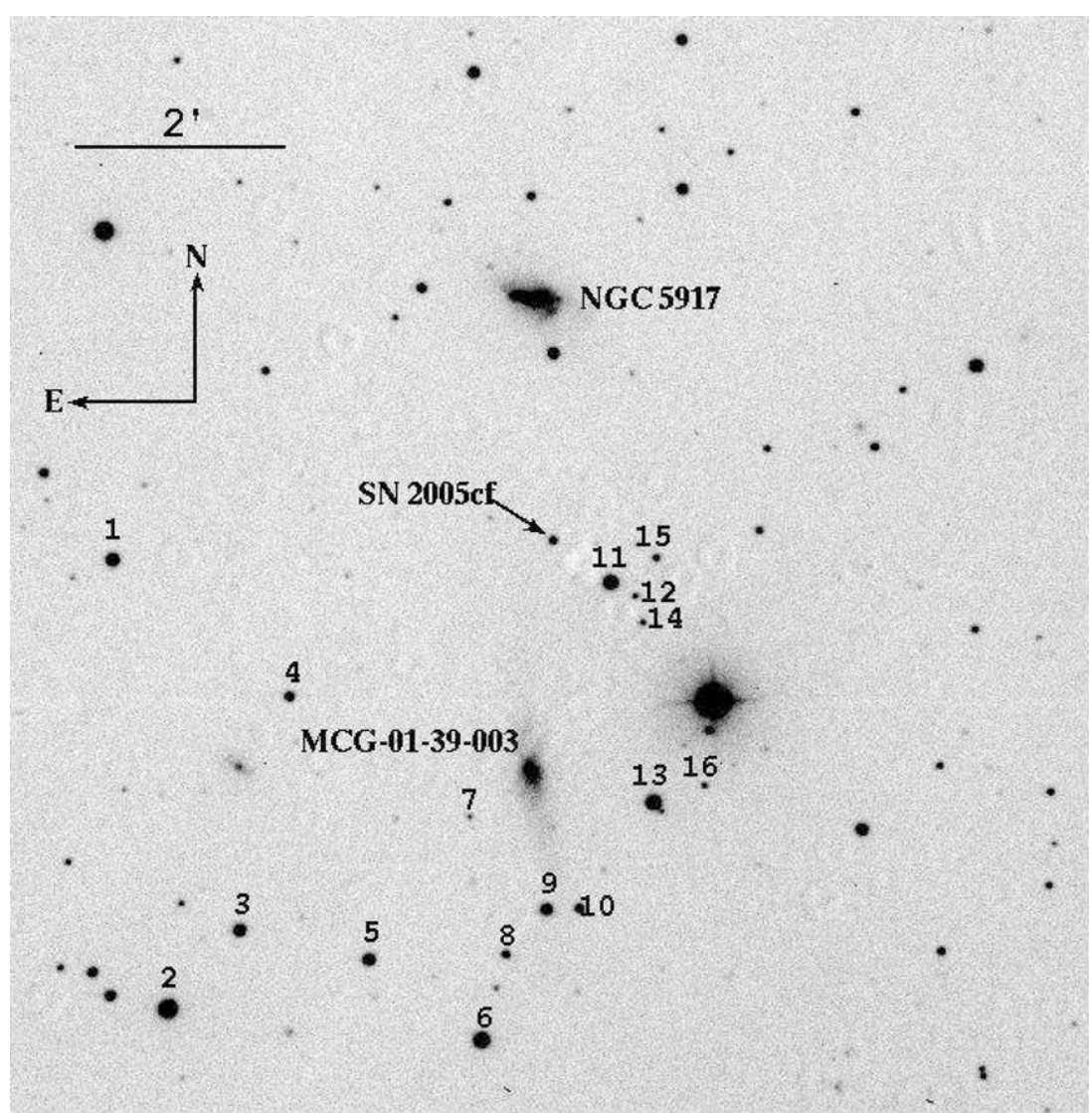

Figure 1. SN 2005cf in MCG-01-39-003. This is a $V$-band image taken with the $0.8 \mathrm{~m}$ TNT on 2005 September 21 . The supernova and 16 local reference stars are marked.

Table 1

Instrumental Color Terms (mag) for Different Telescopes

\begin{tabular}{|c|c|c|c|c|c|}
\hline Telescope & $U(u-b)$ & $B(b-v)$ & $V(b-v)$ & $R(v-r)$ & $I(v-i)$ \\
\hline KAIT $0.76 \mathrm{~m}$ & $0.093(0.017)$ & $0.047(0.011)$ & $-0.038(0.007)$ & $-0.075(0.012)$ & $0.010(0.006)$ \\
\hline FLWO $1.2 \mathrm{~m}$ & $0.038(0.003)$ & $0.091(0.003)$ & $-0.044(0.002)$ & $-0.261(0.010)$ & $-0.122(0.008)$ \\
\hline CTIO $1.3 \mathrm{~m}$ & $\cdots$ & $0.038(0.001)$ & $-0.053(0.005)$ & $-0.030(0.009)$ & $-0.075(0.006)$ \\
\hline Palomar $1.5 \mathrm{~m}$ & $\cdots$ & $0.114(0.011)$ & $-0.023(0.007)$ & $-0.075(0.012)$ & $-0.042(0.006)$ \\
\hline Lick $1.0 \mathrm{~m}$ & $0.087(0.010)$ & $0.093(0.011)$ & $-0.070(0.007)$ & $-0.111(0.012)$ & $0.034(0.006)$ \\
\hline Liverpool $2.0 \mathrm{~m}$ & $\cdots$ & $0.050(0.004)$ & $-0.060(0.005)$ & $-0.250(0.008)$ & $-0.111(0.004)$ \\
\hline
\end{tabular}

Note. Uncertainties are $1 \sigma$.

the point-spread function (PSF) fitting method, performed using the IRAF ${ }^{16}$ DAOPHOT package (Stetson 1987).

The transformation from the instrumental magnitudes to the standard Johnson $U B V$ (Johnson et al. 1966) and KronCousins RI (Cousins 1981) systems was established by using the following equations:

$$
\begin{gathered}
U=u-K_{U} X+C T_{U}(u-b)+Z P_{U}, \\
B=b-K_{B} X+C T_{B}(b-v)+Z P_{B}, \\
V=v-K_{V} X+C T_{V}(b-v)+Z P_{V},
\end{gathered}
$$

16 IRAF, the Image Reduction and Analysis Facility, is distributed by the National Optical Astronomy Observatory, which is operated by the Association of Universities for Research in Astronomy (AURA), Inc. under cooperative agreement with the National Science Foundation (NSF).

$$
\begin{gathered}
R=r-K_{R} X+C T_{R}(b-v)+Z P_{R}, \text { and } \\
I=i-K_{I} X+C T_{I}(b-v)+Z P_{I},
\end{gathered}
$$

where $U B V R I$ are magnitudes in the standard system, ubvri are instrumental magnitudes, and $X$ is the air mass. The coefficients $K_{i}, C T_{i}$, and $Z P_{i}$ refer, respectively, to the atmospheric extinction coefficient, the color terms, and the zero points of the transformation between the instrumental and standard magnitudes. These parameters were determined by observing, during photometric nights, a series of Landolt (1992) standards covering a large range of air masses and colors. The color terms for all of the involved telescopes and filters are listed in Table 1. The average value of the photometric zero points determined for the KAIT on seven photometric nights was used to calibrate the local standard stars in the field of SN 2005cf. Table 2 lists the standard $U B V R I$ magnitudes and uncertainties of 16 
Table 2

Photometric Standards in the SN 2005cf Field ${ }^{\mathrm{a}}$

\begin{tabular}{|c|c|c|c|c|c|}
\hline Star & $U(\mathrm{mag})$ & $B$ (mag) & $V(\mathrm{mag})$ & $R$ (mag) & $I$ (mag) \\
\hline 1 & $\cdots$ & $15.265(0.010)$ & $14.380(0.007)$ & $13.877(0.006)$ & $13.493(0.093)$ \\
\hline 2 & $13.649(0.021)$ & $13.486(0.012)$ & $12.799(0.013)$ & $12.417(0.076)$ & $12.035(0.017)$ \\
\hline 3 & $16.180(0.021)$ & $15.625(0.008)$ & $14.676(0.012)$ & $14.086(0.002)$ & $13.560(0.008)$ \\
\hline 4 & $16.540(0.043)$ & $16.466(0.008)$ & $15.756(0.007)$ & $15.349(0.008)$ & $14.947(0.014)$ \\
\hline 5 & $15.343(0.032)$ & $15.328(0.011)$ & $14.820(0.011)$ & $14.519(0.005)$ & $14.199(0.002)$ \\
\hline 6 & $13.994(0.014)$ & $14.059(0.027)$ & $13.604(0.014)$ & $13.329(0.005)$ & $13.037(0.019)$ \\
\hline 7 & $18.297(0.030)$ & $18.434(0.017)$ & $17.786(0.001)$ & $17.518(0.020)$ & $17.156(0.025)$ \\
\hline 8 & $18.926(0.030)$ & $17.810(0.020)$ & $16.264(0.007)$ & $15.236(0.007)$ & $14.048(0.007)$ \\
\hline 9 & $15.783(0.048)$ & $15.660(0.023)$ & $14.986(0.010)$ & $14.597(0.007)$ & $14.248(0.023)$ \\
\hline 10 & $18.294(0.030)$ & $17.124(0.016)$ & $15.947(0.011)$ & $15.155(0.012)$ & $14.488(0.018)$ \\
\hline 11 & $14.862(0.046)$ & $14.747(0.008)$ & $14.022(0.005)$ & $13.594(0.001)$ & $13.201(0.017)$ \\
\hline 12 & $19.353(0.030)$ & $18.338(0.012)$ & $17.327(0.017)$ & $16.708(0.009)$ & $16.205(0.025)$ \\
\hline 13 & $15.240(0.051)$ & $14.760(0.006)$ & $13.883(0.006)$ & $13.337(0.005)$ & $12.823(0.015)$ \\
\hline 14 & $18.558(0.030)$ & $18.139(0.025)$ & $17.393(0.027)$ & $16.925(0.030)$ & $16.451(0.019)$ \\
\hline 15 & $18.297(0.030)$ & $17.591(0.011)$ & $16.715(0.007)$ & $16.187(0.005)$ & $15.756(0.011)$ \\
\hline 16 & $17.933(0.030)$ & $17.981(0.020)$ & $17.450(0.015)$ & 17.107(0.039) & $16.724(0.074)$ \\
\hline
\end{tabular}

Notes. Uncertainties are $1 \sigma$.

a See Figure 1 for a chart of SN 2005cf and the comparison stars.

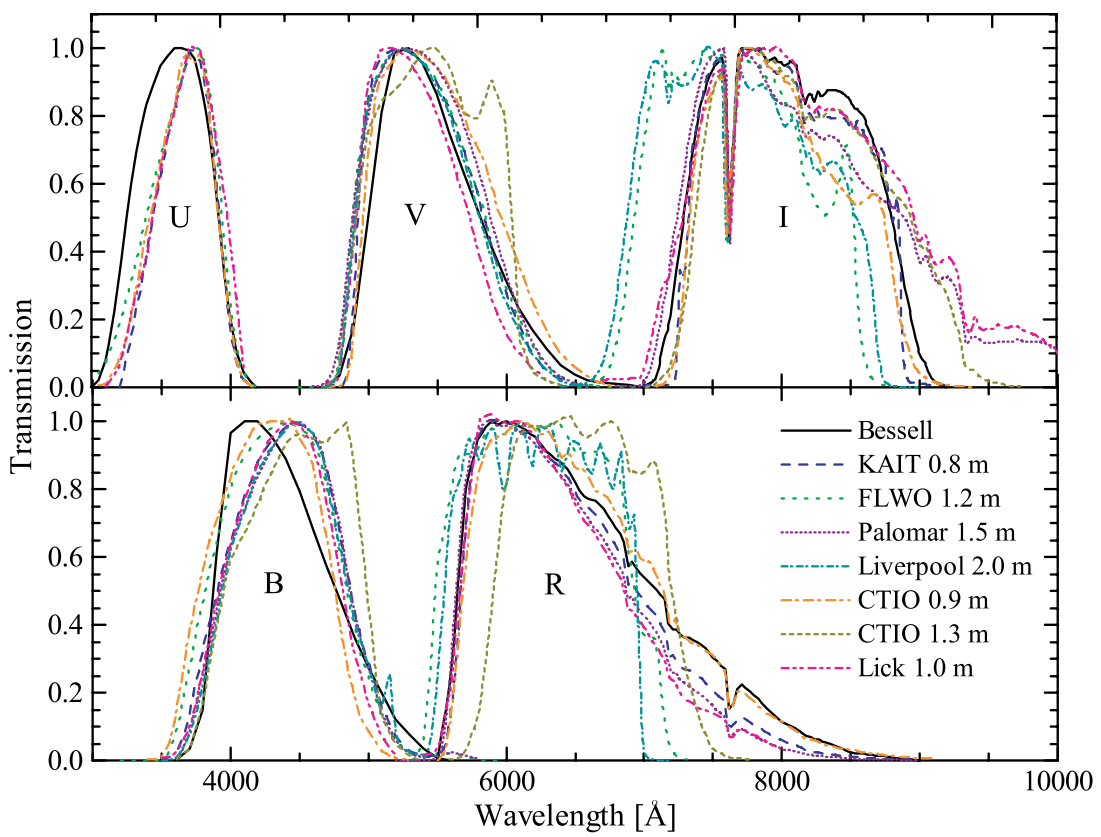

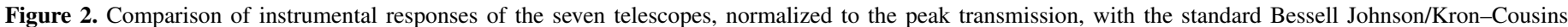
functions.

(A color version of this figure is available in the online journal.)

comparison stars which were used to convert the instrumental magnitudes of the SN to those of the standard system. Note, however, that our $U$-band calibration may have an uncertainty larger than that quoted in Table 2, as it was established on only three photometric nights. The $U B V$ calibrations of SN 2005cf were also used by Li et al. (2006) to calibrate the Swift UVOT optical observations.

A comparison of eight standard stars in common with P07 reveals that some systematic differences exist between the data sets. With respect to $\mathrm{P} 07$, our measurements are fainter by $0.123 \pm 0.033 \mathrm{mag}$ in $U, 0.032 \pm 0.009 \mathrm{mag}$ in $B, 0.040 \pm 0.017$ mag in $V, 0.043 \pm 0.007 \mathrm{mag}$ in $R$, and $0.029 \pm 0.007 \mathrm{mag}$ in $I$. The discrepancies are non-negligible and worrisome, especially in the $U$ band. Such differences were also noticed by Stanishev et al. (2007) in studying photometry of the comparison stars of
SN 2003du; their measurements of the stars in common were found to be systematically brighter than those given by Leonard et al. (2005) and Anupama et al. (2005) by 0.04-0.06 mag in some passbands. The origin of these differences is unclear, and further studies are needed if systematic errors are to be minimized (to $\lesssim 0.01 \mathrm{mag}$ ) when using SNe Ia to measure cosmological distances.

To reasonably assemble the optical photometric data for SN $2005 \mathrm{cf}$ obtained with different telescopes and place them on the Johnson-Cousins UVBRI system (e.g., from Sloan ri to broadband $R I$ ), we applied additional magnitude corrections ( $S$-corrections; Stritzinger et al. 2002) to the photometry. This is because the color-term corrections only account for the differences derived from normal stars, whereas the spectra of SNe are quite dissimilar. Properly modeling the instrumental response is 

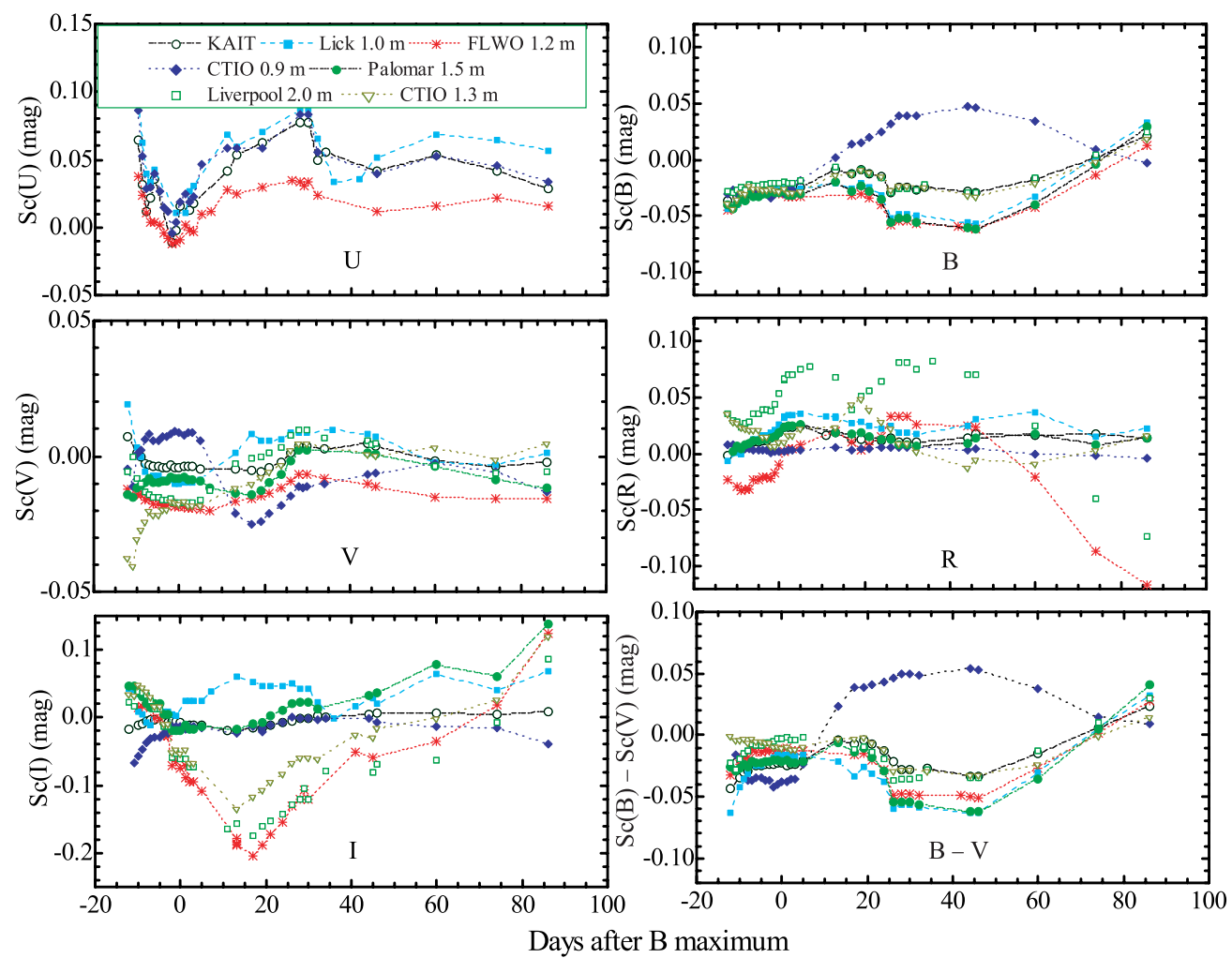

Figure 3. Time evolution of the $S$-corrections at the various telescopes.

(A color version of this figure is available in the online journal.)

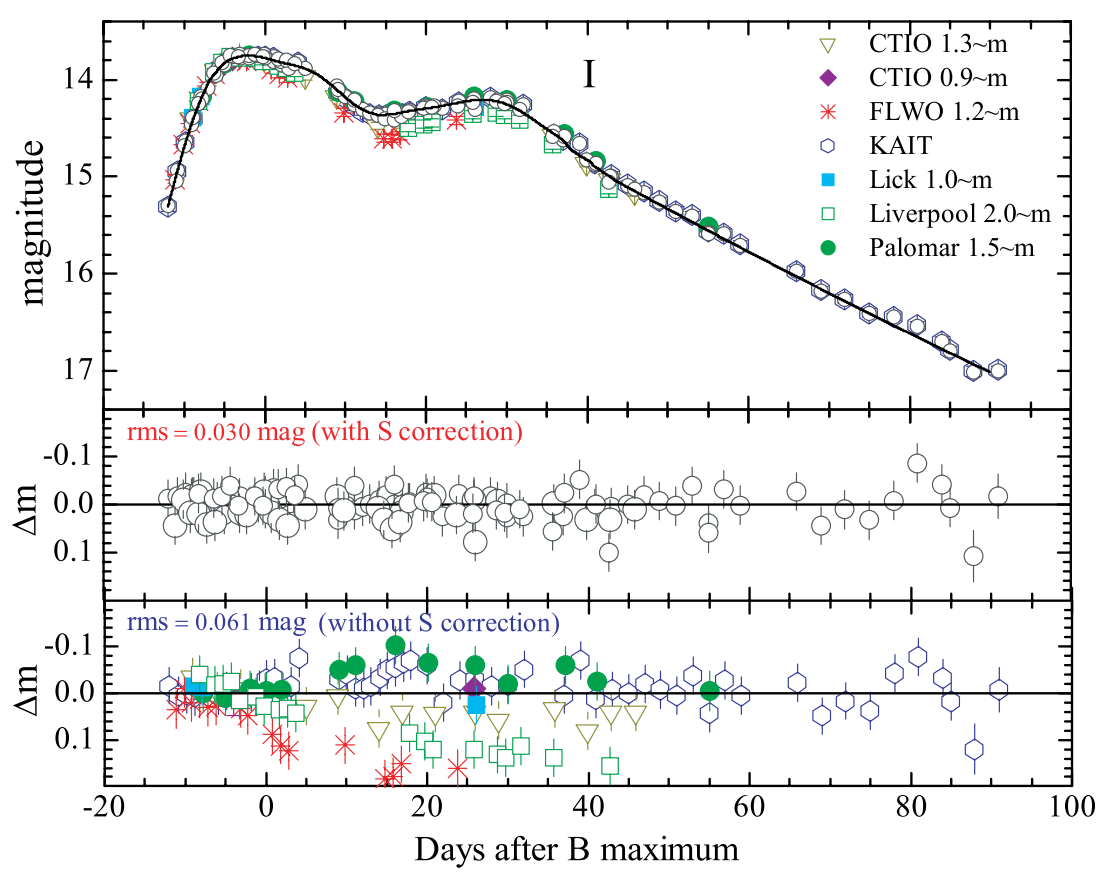

Figure 4. $I$-band light curve of SN $2005 \mathrm{cf}$ with and without application of the $S$-corrections. The solid line in the upper panel represents the best fit to the $S$-corrected light curve.

(A color version of this figure is available in the online journal.)

essential for deriving reliable $S$-corrections. The normalized instrumental response functions, obtained by multiplying the filter transmission functions by the quantum efficiency of the CCD detectors and the atmospheric transmission, are shown in Figure 2. Details of the application of the $S$-corrections are given in the Appendix.
Figure 3 shows the time evolution of the $S$-corrections and the resulting color variances for different telescopes, computed with the spectra of SN $2005 \mathrm{cf}$ presented in this paper and those published by G07, as well as with some late-time spectra of SN 2003du (Stanishev et al. 2007). Note that all of the $U$-band filter responses were cut off at $3300 \AA$ in the convolution due to 
Table 3

$K$-Corrected and S-Corrected Optical Photometry of SN 2005cf

\begin{tabular}{|c|c|c|c|c|c|c|c|c|}
\hline UT Date & JD-2,450,000 & Phase $^{\mathrm{a}}$ & $U(\mathrm{mag})$ & $B$ (mag) & $V(\mathrm{mag})$ & $R(\mathrm{mag})$ & $I$ (mag) & Instrument $^{\mathrm{b}}$ \\
\hline 2005 May 31 & 3521.75 & -11.91 & $15.831(0.038)$ & $15.600(0.022)$ & $15.346(0.022)$ & $15.189(0.032)$ & $15.360(0.041)$ & 2 \\
\hline 2005 May 31 & 3521.77 & -11.89 & $15.819(0.038)$ & $15.579(0.029)$ & $15.293(0.024)$ & $15.206(0.041)$ & $15.312(0.041)$ & 1 \\
\hline 2005 Jun 1 & 3522.74 & -10.92 & $15.329(0.035)$ & $15.151(0.022)$ & $15.000(0.022)$ & $14.838(0.032)$ & $15.062(0.041)$ & 2 \\
\hline 2005 Jun 1 & 3522.87 & -10.79 & $15.223(0.035)$ & $15.096(0.022)$ & $14.955(0.022)$ & $14.846(0.032)$ & $14.953(0.041)$ & 1 \\
\hline 2005 Jun 1 & 3523.15 & -10.51 & $\ldots$ & $15.054(0.041)$ & $14.954(0.032)$ & $14.793(0.032)$ & $14.896(0.041)$ & 3 \\
\hline 2005 Jun 2 & 3523.77 & -9.89 & $14.820(0.035)$ & $14.774(0.022)$ & $14.721(0.022)$ & $14.474(0.032)$ & $14.701(0.041)$ & 2 \\
\hline 2005 Jun 2 & 3523.87 & -9.79 & $14.737(0.035)$ & $14.765(0.022)$ & $14.670(0.022)$ & $14.536(0.032)$ & $14.651(0.041)$ & 1 \\
\hline 2005 Jun 2 & 3524.13 & -9.53 & $\ldots$ & $14.751(0.022)$ & $14.654(0.022)$ & $14.488(0.032)$ & $14.573(0.041)$ & 3 \\
\hline 2005 Jun 3 & 3524.63 & -9.03 & $\cdots$ & $14.587(0.022)$ & $14.497(0.022)$ & $14.411(0.032)$ & $14.464(0.041)$ & 4 \\
\hline 2005 Jun 3 & 3524.68 & -8.98 & $\ldots$ & $14.543(0.022)$ & $14.502(0.022)$ & $14.291(0.032)$ & $14.487(0.041)$ & 2 \\
\hline 2005 Jun 3 & 3524.79 & -8.87 & $\ldots$ & $14.512(0.022)$ & $14.489(0.022)$ & $14.326(0.032)$ & $14.409(0.041)$ & 5 \\
\hline 2005 Jun 3 & 3524.85 & -8.81 & $14.349(0.035)$ & $14.494(0.022)$ & $14.438(0.022)$ & $14.298(0.032)$ & $14.407(0.041)$ & 1 \\
\hline 2005 Jun 3 & 3525.42 & -8.24 & $\cdots$ & $14.343(0.024)$ & $14.344(0.022)$ & $14.190(0.032)$ & $14.269(0.041)$ & 6 \\
\hline 2005 Jun 4 & 3525.69 & -7.97 & $14.123(0.035)$ & $14.304(0.022)$ & $14.293(0.022)$ & $14.079(0.032)$ & $14.259(0.041)$ & 2 \\
\hline 2005 Jun 4 & 3525.76 & -7.90 & $\ldots$ & $14.293(0.022)$ & $14.299(0.022)$ & $14.128(0.032)$ & $14.199(0.041)$ & 5 \\
\hline 2005 Jun 4 & 3525.87 & -7.79 & $\ldots$ & $14.274(0.022)$ & $14.247(0.022)$ & $14.095(0.032)$ & $14.217(0.041)$ & 7 \\
\hline 2005 Jun 5 & 3526.68 & -6.98 & $\cdots$ & $14.127(0.022)$ & $14.122(0.022)$ & $13.916(0.032)$ & $14.108(0.041)$ & 2 \\
\hline 2005 Jun 5 & 3526.75 & -6.91 & $13.852(0.035)$ & $14.101(0.022)$ & $14.118(0.022)$ & $\ldots$ & $\ldots$ & 5 \\
\hline 2005 Jun 5 & 3527.44 & -6.22 & $\ldots$ & $14.007(0.022)$ & $14.003(0.022)$ & $13.875(0.032)$ & $13.948(0.041)$ & 6 \\
\hline 2005 Jun 6 & 3527.64 & -6.02 & $\cdots$ & $14.014(0.022)$ & $13.992(0.022)$ & $13.884(0.032)$ & $13.948(0.041)$ & 4 \\
\hline 2005 Jun 6 & 3527.69 & -5.97 & $13.703(0.035)$ & $13.979(0.022)$ & $13.981(0.022)$ & $13.785(0.032)$ & $13.970(0.041)$ & 2 \\
\hline 2005 Jun 6 & 3527.85 & -5.81 & $13.624(0.035)$ & $13.950(0.022)$ & $13.942(0.022)$ & $13.796(0.032)$ & $13.915(0.041)$ & 1 \\
\hline 2005 Jun 6 & 3528.43 & -5.23 & $\ldots$ & $13.863(0.022)$ & $13.896(0.022)$ & $13.781(0.032)$ & $13.845(0.041)$ & 6 \\
\hline 2005 Jun 7 & 3528.75 & -4.91 & $\ldots$ & $13.861(0.022)$ & $13.852(0.022)$ & $13.708(0.032)$ & $13.863(0.041)$ & 7 \\
\hline 2005 Jun 7 & 3528.84 & -4.82 & $13.533(0.035)$ & $13.843(0.022)$ & $13.843(0.022)$ & $13.697(0.032)$ & $13.828(0.041)$ & 1 \\
\hline 2005 Jun 8 & 3529.43 & -4.23 & $\ldots$ & $13.755(0.025)$ & $13.795(0.022)$ & $13.704(0.032)$ & $13.765(0.041)$ & 6 \\
\hline 2005 Jun 8 & 3529.71 & -3.95 & $13.496(0.035)$ & $13.768(0.022)$ & $13.783(0.022)$ & $13.683(0.032)$ & $13.778(0.041)$ & 8 \\
\hline 2005 Jun 8 & 3530.42 & -3.24 & $\ldots$ & $13.716(0.022)$ & $13.736(0.022)$ & $13.629(0.032)$ & $13.774(0.041)$ & 6 \\
\hline 2005 Jun 8 & 3530.59 & -3.07 & $\ldots$ & $13.741(0.022)$ & $13.712(0.022)$ & $13.644(0.032)$ & $13.755(0.041)$ & 4 \\
\hline 2005 Jun 9 & 3530.68 & -2.98 & $13.402(0.035)$ & $13.718(0.022)$ & $13.713(0.022)$ & $13.558(0.032)$ & $13.786(0.041)$ & 2 \\
\hline 2005 Jun 10 & 3531.67 & -1.99 & $13.364(0.035)$ & $13.677(0.022)$ & $13.655(0.022)$ & $13.523(0.032)$ & $13.783(0.041)$ & 2 \\
\hline 2005 Jun 10 & 3531.79 & -1.87 & $\cdots$ & $13.666(0.022)$ & $13.630(0.022)$ & $13.551(0.032)$ & $13.767(0.041)$ & 7 \\
\hline 2005 Jun 10 & 3531.83 & -1.83 & $13.391(0.035)$ & $13.664(0.022)$ & $13.639(0.025)$ & $13.577(0.032)$ & $13.767(0.041)$ & 1 \\
\hline 2005 Jun 10 & 3532.42 & -1.24 & $\ldots$ & $13.654(0.022)$ & $13.638(0.022)$ & $13.550(0.032)$ & $13.745(0.041)$ & 6 \\
\hline 2005 Jun 11 & 3532.87 & -0.79 & $13.372(0.035)$ & $13.643(0.022)$ & $13.600(0.022)$ & $13.548(0.032)$ & $13.766(0.041)$ & 1 \\
\hline 2005 Jun 11 & 3533.42 & -0.24 & $\ldots$ & $13.621(0.021)$ & $13.595(0.022)$ & $13.559(0.032)$ & $13.772(0.041)$ & 6 \\
\hline 2005 Jun 12 & 3533.66 & 0 & $\cdots$ & $13.650(0.022)$ & $13.592(0.022)$ & $13.578(0.032)$ & $13.748(0.041)$ & 4 \\
\hline 2005 Jun 12 & 3533.72 & 0.06 & $\ldots$ & $13.623(0.022)$ & $13.570(0.022)$ & $13.515(0.032)$ & $13.785(0.041)$ & 7 \\
\hline 2005 Jun 12 & 3533.84 & 0.18 & $13.362(0.035)$ & $13.619(0.022)$ & $13.568(0.022)$ & $13.517(0.032)$ & $13.761(0.041)$ & 1 \\
\hline 2005 Jun 13 & 3534.73 & 1.07 & $13.413(0.035)$ & $13.637(0.022)$ & $13.574(0.022)$ & $13.485(0.032)$ & $13.821(0.041)$ & 2 \\
\hline 2005 Jun 13 & 3534.84 & 1.18 & $13.381(0.035)$ & $13.622(0.022)$ & $13.549(0.022)$ & $13.525(0.032)$ & $13.773(0.041)$ & 1 \\
\hline 2005 Jun 13 & 3535.43 & 1.77 & $\ldots$ & $13.649(0.022)$ & $13.581(0.022)$ & $13.540(0.032)$ & $13.787(0.041)$ & 6 \\
\hline 2005 Jun 14 & 3535.72 & 2.06 & $\cdots$ & $13.641(0.022)$ & $13.564(0.022)$ & $13.505(0.032)$ & $13.812(0.041)$ & 7 \\
\hline 2005 Jun 14 & 3535.74 & 2.08 & $13.458(0.035)$ & $13.654(0.022)$ & $13.574(0.022)$ & $13.496(0.032)$ & $13.858(0.041)$ & 2 \\
\hline 2005 Jun 14 & 3535.83 & 2.17 & $13.456(0.035)$ & $13.631(0.022)$ & $13.556(0.032)$ & $13.509(0.032)$ & $\cdots$ & 1 \\
\hline 2005 Jun 14 & 3536.44 & 2.78 & $\cdots$ & $13.719(0.021)$ & $13.591(0.022)$ & $13.539(0.032)$ & $13.800(0.041)$ & 6 \\
\hline 2005 Jun 15 & 3536.70 & 3.04 & $13.533(0.035)$ & $13.702(0.022)$ & $13.581(0.022)$ & $13.519(0.032)$ & $13.884(0.041)$ & 2 \\
\hline 2005 Jun 15 & 3536.83 & 3.17 & $13.532(0.035)$ & $13.681(0.022)$ & $13.558(0.022)$ & $13.545(0.032)$ & $13.840(0.041)$ & 1 \\
\hline 2005 Jun 15 & 3537.47 & 3.81 & $\cdots$ & $13.717(0.022)$ & $13.616(0.022)$ & $13.551(0.032)$ & $13.833(0.041)$ & 6 \\
\hline 2005 Jun 16 & 3537.82 & 4.16 & $\ldots$ & $\ldots$ & $13.554(0.022)$ & $13.527(0.032)$ & $13.814(0.041)$ & 1 \\
\hline 2005 Jun 17 & 3538.68 & 5.02 & $\cdots$ & $13.815(0.022)$ & $13.624(0.022)$ & $13.638(0.032)$ & $13.884(0.041)$ & 4 \\
\hline 2005 Jun 21 & 3542.61 & 8.95 & $\cdots$ & $14.093(0.045)$ & $13.733(0.022)$ & $13.813(0.032)$ & $14.059(0.041)$ & 4 \\
\hline 2005 Jun 21 & 3542.72 & 9.06 & $14.086(0.054)$ & $14.090(0.022)$ & $13.740(0.022)$ & $13.789(0.032)$ & $14.117(0.041)$ & 1 \\
\hline 2005 Jun 21 & 3542.76 & 9.10 & $\ldots$ & $14.040(0.035)$ & $13.709(0.033)$ & $13.742(0.032)$ & $14.093(0.041)$ & 7 \\
\hline 2005 Jun 21 & 3543.06 & 9.40 & $\cdots$ & $\cdots$ & $13.717(0.031)$ & $13.795(0.032)$ & $14.143(0.053)$ & 3 \\
\hline 2005 Jun 22 & 3543.68 & 10.02 & $14.185(0.035)$ & $14.171(0.022)$ & $13.797(0.022)$ & $13.814(0.032)$ & $14.171(0.041)$ & 2 \\
\hline 2005 Jun 22 & 3543.82 & 10.16 & $14.188(0.035)$ & $14.182(0.022)$ & $13.808(0.022)$ & $13.868(0.032)$ & $14.168(0.041)$ & 1 \\
\hline 2005 Jun 23 & 3544.77 & 11.11 & $\cdots$ & $14.217(0.021)$ & $13.867(0.027)$ & $13.888(0.032)$ & $14.197(0.041)$ & 7 \\
\hline 2005 Jun 23 & 3544.80 & 11.14 & $14.268(0.035)$ & $14.278(0.023)$ & $13.866(0.032)$ & $13.941(0.035)$ & $14.248(0.041)$ & 1 \\
\hline 2005 Jun 24 & 3545.82 & 12.16 & $14.398(0.035)$ & $14.377(0.022)$ & $13.894(0.029)$ & $14.013(0.032)$ & $14.297(0.041)$ & 1 \\
\hline 2005 Jun 25 & 3546.75 & 13.09 & $14.508(0.035)$ & $14.465(0.026)$ & $13.946(0.034)$ & $14.054(0.034)$ & $14.328(0.041)$ & 1 \\
\hline 2005 Jun 26 & 3547.67 & 14.01 & $\cdots$ & $14.553(0.022)$ & $14.015(0.022)$ & $14.141(0.032)$ & $14.336(0.041)$ & 4 \\
\hline 2005 Jun 28 & 3549.74 & 16.08 & $\ldots$ & $14.755(0.022)$ & $14.118(0.023)$ & $14.168(0.032)$ & $14.309(0.041)$ & 7 \\
\hline 2005 Jun 28 & 3549.79 & 16.13 & $14.952(0.035)$ & $14.781(0.022)$ & $14.119(0.029)$ & $14.200(0.039)$ & $14.349(0.041)$ & 1 \\
\hline 2005 Jun 29 & 3550.66 & 17.00 & $15.110(0.035)$ & $14.879(0.022)$ & $14.178(0.022)$ & $14.161(0.032)$ & $14.371(0.041)$ & 2 \\
\hline 2005 Jun 29 & 3550.67 & 17.01 & $\ldots$ & $14.911(0.022)$ & $14.195(0.022)$ & $14.251(0.032)$ & $14.340(0.041)$ & 4 \\
\hline
\end{tabular}


Table 3

(Continued)

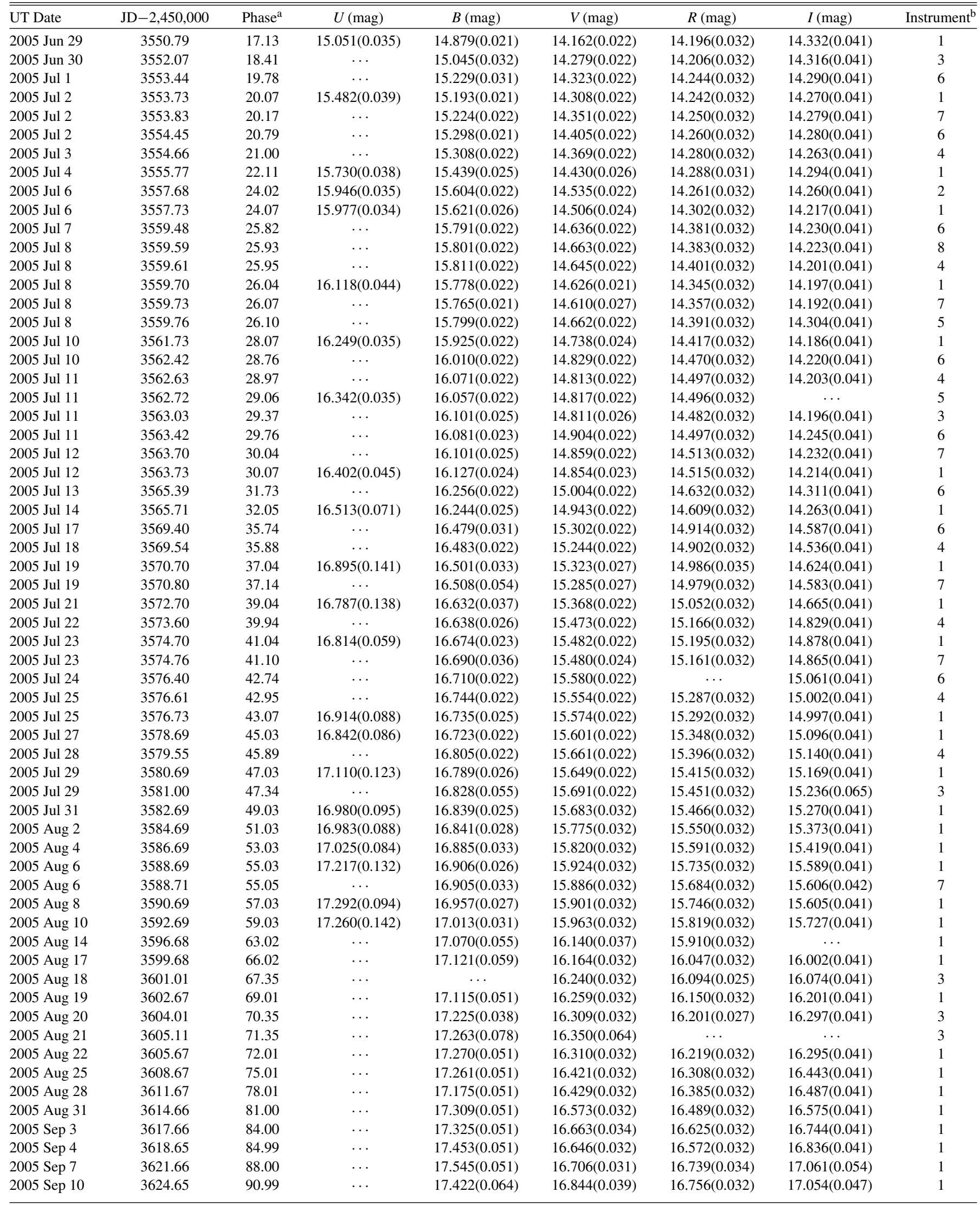

Notes. Uncertainties are $1 \sigma$.

a Relative to the epoch of $B$-band maximum (JD $=2,453,533.66)$.

b $1=$ KAIT $0.76 \mathrm{~m} ; 2=$ FLWO $1.2 \mathrm{~m} ; 3=$ TNT $0.8 \mathrm{~m} ; 4=$ CTIO $1.3 \mathrm{~m} ; 5=$ Lick $1.0 \mathrm{~m} ; 6=$ Liverpool $2.0 \mathrm{~m} ; 7=$ Palomar $1.5 \mathrm{~m} ; 8=$ CTIO $0.9 \mathrm{~m}$. 
Table 4

$J H K_{s}$ Magnitudes of SN 2005cf from PAIRITEL

\begin{tabular}{|c|c|c|c|c|c|c|c|c|}
\hline UT Date & $\mathrm{JD}-2,450,000$ & Phase $^{\mathrm{a}}$ & $J$ & $H$ & $K_{s}$ & $K_{J}$ & $K_{H}$ & $K_{K_{s}}$ \\
\hline 2005 May 31 & 3522.24 & -11.42 & $14.724(0.023)$ & $14.689(0.019)$ & $14.825(0.021)$ & 0.015 & 0.010 & 0.016 \\
\hline 2005 Jun 1 & 3523.25 & -10.41 & $14.495(0.015)$ & $14.514(0.031)$ & $\cdots$ & 0.015 & 0.010 & $\cdots$ \\
\hline 2005 Jun 2 & 3524.24 & -9.42 & $14.254(0.028)$ & $14.316(0.031)$ & $14.347(0.021)$ & 0.015 & 0.010 & 0.016 \\
\hline 2005 Jun 10 & 3532.23 & -1.43 & $13.841(0.039)$ & $13.969(0.023)$ & $14.002(0.023)$ & 0.011 & 0.006 & 0.026 \\
\hline 2005 Jun 12 & 3534.23 & 0.57 & $14.035(0.044)$ & $14.040(0.036)$ & $13.995(0.015)$ & 0.010 & 0.004 & 0.030 \\
\hline 2005 Jun 13 & 3535.22 & 1.56 & 13.997(0.013) & $14.142(0.014)$ & $14.102(0.034)$ & 0.009 & -0.003 & 0.034 \\
\hline 2005 Jun 14 & 3536.21 & 2.55 & $14.076(0.014)$ & $14.154(0.034)$ & $14.093(0.019)$ & 0.008 & -0.006 & 0.038 \\
\hline 2005 Jun 17 & 3539.21 & 5.55 & $14.346(0.023)$ & $14.169(0.056)$ & $14.184(0.029)$ & 0.006 & -0.010 & 0.046 \\
\hline 2005 Jun 29 & 3551.16 & 17.50 & $15.365(0.025)$ & $13.974(0.064)$ & $14.288(0.019)$ & 0.022 & -0.021 & 0.037 \\
\hline 2005 Jul 1 & 3553.17 & 19.51 & $15.339(0.028)$ & $13.943(0.063)$ & $14.196(0.017)$ & 0.025 & -0.021 & 0.032 \\
\hline 2005 Jul 2 & 3554.17 & 20.51 & $15.333(0.023)$ & $14.060(0.033)$ & $\cdots$ & 0.027 & -0.022 & $\cdots$ \\
\hline 2005 Jul 3 & 3555.28 & 21.62 & $15.294(0.018)$ & $13.979(0.060)$ & $14.181(0.017)$ & 0.029 & -0.023 & 0.026 \\
\hline $2005 \mathrm{Jul} 4$ & 3556.17 & 22.51 & $15.261(0.023)$ & $14.040(0.017)$ & $14.167(0.021)$ & 0.030 & -0.024 & 0.023 \\
\hline 2005 Jul 5 & 3557.15 & 23.49 & $15.174(0.023)$ & $13.984(0.081)$ & $14.101(0.011)$ & 0.033 & -0.022 & 0.019 \\
\hline $2005 \mathrm{Jul} 6$ & 3558.15 & 24.49 & $15.191(0.036)$ & $13.983(0.089)$ & $14.105(0.023)$ & 0.036 & -0.021 & 0.016 \\
\hline 2005 Jul 8 & 3560.16 & 26.50 & $14.995(0.026)$ & $14.017(0.018)$ & $14.123(0.021)$ & 0.041 & -0.017 & 0.008 \\
\hline 2005 Jul 11 & 3563.15 & 29.49 & $14.846(0.024)$ & $14.087(0.021)$ & $14.219(0.019)$ & 0.041 & -0.008 & 0.005 \\
\hline
\end{tabular}

Notes. The $K$-corrections listed in Columns 7-9 were added to the $J H K s$ magnitudes. Uncertainties are $1 \sigma$.

${ }^{\text {a }}$ Relative to the epoch of $B$-band maximum $(\mathrm{JD}=2,453,533.66)$.

the spectral coverage. The resulting $S$-corrections are generally small in the $B V R$ bands, but can be noticeably large in the $U$ and $I$ bands (e.g., $\sim 0.1-0.2 \mathrm{mag}$ ). It is worth noting that, without applying such a systematic magnitude correction, the $B-V$ color measured at the CTIO $0.9 \mathrm{~m}$ and Palomar $1.5 \mathrm{~m}$ (or Lick $1.0 \mathrm{~m}$ ) telescopes could differ by $\sim 0.1 \mathrm{mag}$ in the early nebular phase when the colors are usually used as reddening indicators.

Applying the $S$-corrections to the photometry noticeably improved the consistency of the data sets obtained with different telescopes. This is demonstrated in Figure 4, where the scatter around the best-fit curve decreases from 0.06 mag to $0.03 \mathrm{mag}$ in the $I$ band. Improvements are also achieved for the other bands, with the photometric scatter reduced to within $0.02-0.03$ mag. Such a normalization in the photometry could be potentially important when comparing the properties of SNe measured with different systems. This is because a shift of a few percent in the $B-V$ color might systematically bias the extinction correction, hence producing an error of $\sim 10 \%$ (a factor of 3-4 larger) in the derived luminosity of the SN. We thus should become fully aware of the possible bias of the reddening inferred from old SN Ia data sets, as the published photometry for most of them has not been $S$-corrected.

We further applied $K$-corrections to our photometry using the same set of spectra as for the $S$-corrections. We note that the $U$-band $K$-correction could reach $\sim 0.06$ mag at the earliest phases, perhaps due to the particular shape of the spectrum at those times. The large $K$ - and $S$-corrections required in the $U$ band, which were usually unavailable due to insufficient spectral coverage, might partially account for the large scatter seen in the $U$-band light curve of SN $2005 \mathrm{cf}$.

The final calibrated $U B V R I$ magnitudes, after performing the $K$ - and $S$-corrections, are presented in Table 3. The error bars (in parentheses) are dominated by the uncertainty in the calibration of the comparison stars.

Since the NIR observations of SN 2005cf were conducted with PAIRITEL, the instrument that defines the Two Micron All Sky Survey (2MASS) photometric system, we use the 2MASS point-source catalog (Cutri et al. 2003) to calibrate the SN. The calibrated $J H K_{s}$ magnitudes of SN $2005 \mathrm{cf}$

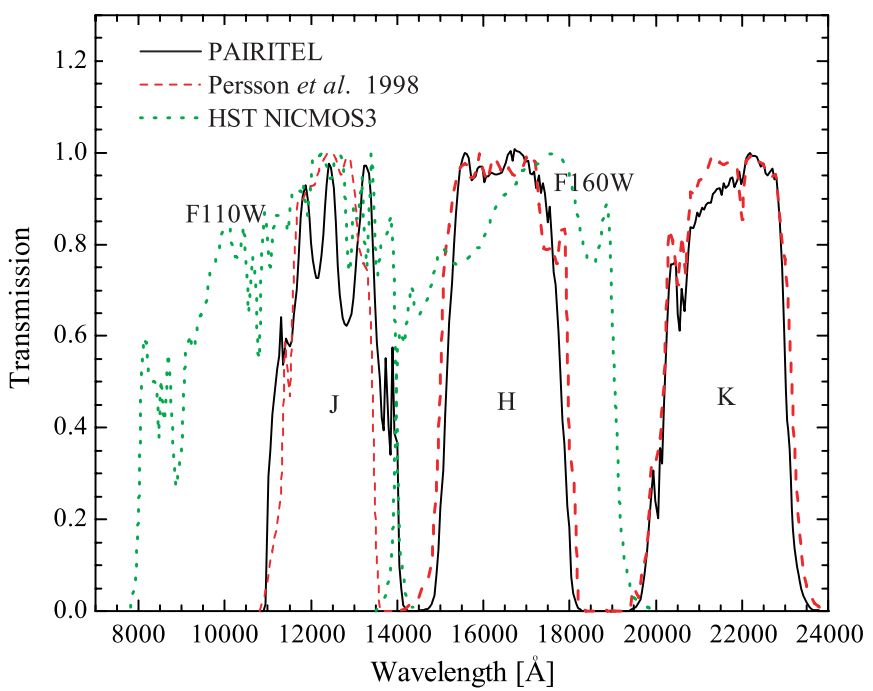

Figure 5. Comparison of near-infrared transmission curves of PAIRITEL (ex2MASS) and HST NICMOS3 with that of the Persson et al. (1998) system.

(A color version of this figure is available in the online journal.)

are given in Table 4, which were $K$-corrected (Columns 7 9) using the NIR spectra of SN 1999ee (Hamuy et al. 2002). No $S$-corrections were applied to the NIR photometry because of the similarity of the transmission curves between the 2MASS system and the Persson et al. (1998) system. The filter transmission curves of these two systems are shown in Figure 5 .

\subsubsection{Optical Spectroscopy}

Low-resolution spectra of SN 2005cf were obtained with the Kast double spectrograph (Miller \& Stone 1993) on the $3.0 \mathrm{~m}$ Shane telescope at Lick Observatory and the FAST spectrograph (Fabricant et al. 1998) on the Tillinghast 1.5 $\mathrm{m}$ telescope at FLWO. Two late-time spectra were also obtained at the W. M. Keck Observatory: one with the Low Resolution Imaging Spectrometer (LRIS; Oke et al. 1995) 
Table 5

Journal of Spectroscopic Observations of SN 2005cf

\begin{tabular}{|c|c|c|c|c|c|c|c|c|}
\hline UT Date & $\mathrm{JD}-2,450,000$ & Phase $^{\mathrm{a}}$ & Range $(\AA)$ & Res. $^{b}(\AA)$ & Air mass & Exp. (s) & Inst. $^{c}$ & Observers \\
\hline 2005 May 31 & 3521.7 & -12.0 & $3480-7500$ & 7 & 1.3 & 1200 & Fast & PB \\
\hline 2005 Jun 1 & 3522.7 & -11.0 & $3480-7500$ & 7 & 1.3 & 960 & Fast & $\mathrm{PB}$ \\
\hline 2005 Jun 1 & 3522.9 & -10.8 & $3300-10400$ & $5-12$ & 1.8 & 600 & Kast & MG,DW,BS \\
\hline 2005 Jun 2 & 3523.7 & -10.0 & $3480-7500$ & 7 & 1.3 & 600 & Fast & $\mathrm{PB}$ \\
\hline 2005 Jun 2 & 3523.9 & -9.8 & $3300-10400$ & $5-12$ & 1.3 & 300 & Kast & DR \\
\hline 2005 Jun 3 & 3524.7 & -9.0 & $3480-7500$ & 7 & 1.3 & 900 & Fast & $\mathrm{MC}$ \\
\hline 2005 Jun 3 & 3524.9 & -8.8 & $3300-10400$ & $5-12$ & 1.4 & 300 & Kast & DR \\
\hline 2005 Jun 4 & 3525.9 & -7.8 & $3300-10400$ & $5-12$ & 1.4 & 600 & Kast & DR \\
\hline 2005 Jun 5 & 3526.9 & -6.8 & $3300-10400$ & $5-12$ & 1.4 & 600 & Kast & DR \\
\hline 2005 Jun 6 & 3527.9 & -5.8 & $3300-10400$ & $5-12$ & 1.4 & 600 & Kast & MM \\
\hline 2005 Jun 7 & 3528.7 & -5.0 & $3480-7500$ & 7 & 1.3 & 900 & Fast & $\mathrm{PB}$ \\
\hline 2005 Jun 8 & 3529.7 & -4.0 & $3480-7500$ & 7 & 1.3 & 900 & Fast & PB \\
\hline 2005 Jun 9 & 3530.7 & -3.0 & $3480-7500$ & 7 & 1.3 & 900 & Fast & PB \\
\hline 2005 Jun 10 & 3531.8 & -1.9 & $3480-7500$ & 7 & 1.7 & 900 & Fast & PB \\
\hline 2005 Jun 10 & 3531.8 & -1.9 & $3300-10400$ & $5-12$ & 1.4 & 300 & Kast & MG,FS \\
\hline 2005 Jun 11 & 3532.7 & -1.0 & $3300-10400$ & $5-12$ & 1.5 & 600 & Kast & FS \\
\hline 2005 Jun 11 & 3532.8 & -0.9 & $3480-7500$ & 7 & 1.5 & 600 & Fast & $\mathrm{MC}$ \\
\hline 2005 Jun 12 & 3533.8 & 0.2 & $3480-7500$ & 7 & 2.1 & 600 & Fast & $\mathrm{MC}$ \\
\hline 2005 Jun 13 & 3534.7 & 1.1 & $3480-7500$ & 7 & 1.4 & 600 & Fast & $\mathrm{PB}$ \\
\hline 2005 Jun 14 & 3535.7 & 2.0 & $3300-10400$ & $5-12$ & 1.4 & 600 & Kast & AF,MG,BS \\
\hline 2005 Jun 14 & 3535.8 & 2.1 & $3480-7500$ & 7 & 1.5 & 600 & Fast & PB \\
\hline 2005 Jun 15 & 3536.7 & 3.0 & $3480-7500$ & 7 & 1.3 & 600 & Fast & PB \\
\hline 2005 Jun 16 & 3537.6 & 3.9 & $3480-7500$ & 7 & 1.3 & 780 & Fast & $\mathrm{MC}$ \\
\hline 2005 Jun 17 & 3538.7 & 5.0 & $3480-7500$ & 7 & 1.3 & 660 & Fast & MC \\
\hline 2005 Jun 29 & 3550.8 & 17.1 & $3480-7500$ & 7 & 2.0 & 900 & Fast & $\mathrm{RH}$ \\
\hline 2005 Jul 1 & 3552.7 & 19.0 & $3300-10400$ & $5-12$ & 1.4 & 300 & Kast & MG,DW \\
\hline $2005 \mathrm{Jul} 4$ & 3555.7 & 22.0 & $3480-7500$ & 7 & 1.4 & 600 & Fast & JG \\
\hline 2005 Jul 6 & 3557.7 & 24.0 & $3480-7500$ & 7 & 1.4 & 900 & Fast & JG \\
\hline $2005 \mathrm{Jul} 7$ & 3558.6 & 24.9 & $3480-7500$ & 7 & 1.3 & 900 & Fast & $\mathrm{EF}$ \\
\hline $2005 \mathrm{Jul} 8$ & 3559.6 & 25.9 & $3480-7500$ & 7 & 1.3 & 900 & Fast & PB \\
\hline $2005 \mathrm{Jul} 9$ & 3560.7 & 27.0 & $3480-7500$ & 7 & 1.5 & 900 & Fast & PB \\
\hline 2005 Jul 10 & 3561.7 & 28.0 & $3480-7500$ & 7 & 1.4 & 900 & Fast & PB \\
\hline 2005 Jul 10 & 3561.7 & 28.0 & $3300-10400$ & $5-12$ & 1.5 & 500 & Kast & AF,FS \\
\hline 2005 Jul 11 & 3562.7 & 29.0 & $3480-7500$ & 7 & 1.4 & 900 & Fast & $\mathrm{MC}$ \\
\hline 2005 Jul 12 & 3563.9 & 30.2 & $3480-7500$ & 7 & 1.3 & 1800 & Fast & $\mathrm{MC}$ \\
\hline 2005 Jul 26 & 3577.6 & 43.9 & $3480-7500$ & 7 & 1.4 & 1800 & Fast & $\mathrm{PB}$ \\
\hline 2005 Jul 28 & 3579.6 & 45.9 & $3480-7500$ & 7 & 1.5 & 1200 & Fast & $\mathrm{PB}$ \\
\hline 2005 Sep 3 & 3616.6 & 82.9 & $3480-7500$ & 7 & 2.2 & 1200 & Fast & $\mathrm{MC}$ \\
\hline 2006 Apr 26 & 3853.1 & 319.4 & $3250-9250$ & 6 & 1.5 & 1200 & LRISb & $\mathrm{AF}, \mathrm{RF}$ \\
\hline 2007 Feb 16 & 4148.1 & 614.4 & $4585-7230$ & 1.3 & 1.3 & 6300 & DEIMOS & AF,JS,RF,RC \\
\hline
\end{tabular}

Notes. Fast = FLWO 1.5 m FAST;Kast= Lick Shane 3 m KAST; LIRSb = Keck I 10 m LRIS-blue; DEIMOS = Keck II 10 m DEIMOS.

$\mathrm{AF}=$ Alex Filippenko; RH = Robert Hutchins; EF = Emilio Falco; JG = Joseph Gallagher; PB = Perry Berlind; MC = Mike Calkins; MG = Mohan Ganeshalingam; DW = Diane Wong; BS = Brandon Swift; DR = David Reitzel; JS = Jeffrey Silverman; MM = Matthew Malkan; FS = Frank Serduke; RC = Ryan Chronock; RF = Ryan Foley.

a Relative to the $B$ maximum ( $\mathrm{DD}=2,453,533.66$ ).

b Approximate spectral resolution.

mounted on the $10 \mathrm{~m}$ Keck I telescope, and the other with the Deep Extragalactic Imaging Multi-Object Spectrograph (DEIMOS; Faber et al. 2003) mounted on the 10 m Keck II telescope. A journal of spectroscopic observations is given in Table 5.

All spectra were reduced using standard IRAF routines (e.g., Foley et al. 2003). For the Lick/Kast observations, flatfields for the red-side spectra were taken at the position of the object to reduce NIR fringing effects. For two Lick/Kast spectra taken on 2005 June 10 and June 11, there was condensation on the redside camera, producing nonstandard and variable absorption features. To compensate for this effect, we created a twodimensional surface map of a flatfield image, and smoothed the surface map to remove any fringing in the flat. We then divided our images by this surface to remove the absorption features from our images. Although this process produced significantly improved spectra, there are still some persistent systematic features in those spectra. The Keck/DEIMOS data were reduced using a modified version of the DEEP pipeline and our own routines as described by Foley et al. (2007).

Flux calibration of the spectra was performed by means of spectrophotometric standard stars observed at similar air mass on the same night as the SN. Using our own IDL routines, the extracted, wavelength-calibrated spectra were corrected for continuum atmospheric extinction using mean extinction curves for FLWO and Lick Observatory; moreover, telluric lines were removed from the data. For all the spectra observed at Lick and FLWO, the slit was always aligned close to the parallactic angle to avoid chromatic effects in the data (Filippenko 1982). 
Table 6

HST ACS Ultraviolet Photometry of SN 2005cf

\begin{tabular}{|c|c|c|c|c|c|}
\hline UT Date & $\mathrm{JD}-2,450,000$ & Phase $^{\mathrm{a}}$ & F220W (mag) & F250W (mag) & F330W (mag) \\
\hline 2005 Jun 3 & 3524.99 & -8.67 & $19.978(0.073)$ & $16.624(0.026)$ & $14.675(0.022)$ \\
\hline 2005 Jun 5 & 3527.26 & -6.40 & $19.024(0.037)$ & $15.638(0.022)$ & $13.766(0.021)$ \\
\hline 2005 Jun 7 & 3529.36 & -4.30 & $18.557(0.043)$ & $15.341(0.022)$ & $13.439(0.022)$ \\
\hline 2005 Jun 11 & 3532.96 & -0.70 & $18.189(0.043)$ & $14.972(0.021)$ & $13.272(0.021)$ \\
\hline 2005 Jun 14 & 3535.95 & 2.28 & $18.160(0.055)$ & $15.026(0.021)$ & $13.395(0.021)$ \\
\hline 2005 Jun 16 & 3537.95 & 4.29 & $18.131(0.034)$ & $15.243(0.022)$ & $13.719(0.022)$ \\
\hline 2005 Jun 21 & 3542.61 & 9.02 & $18.240(0.023)$ & $15.941(0.022)$ & $14.424(0.021)$ \\
\hline 2005 Jun 25 & 3547.28 & 13.62 & $18.665(0.023)$ & $16.589(0.022)$ & $15.051(0.021)$ \\
\hline 2005 Jun 26 & 3547.98 & 14.32 & $18.745(0.022)$ & $16.745(0.022)$ & $15.259(0.022)$ \\
\hline 2005 Jun 29 & 3551.28 & 17.68 & $19.060(0.024)$ & $17.068(0.022)$ & $15.677(0.021)$ \\
\hline 2005 Jun 30 & 3551.77 & 18.11 & $19.158(0.023)$ & $17.162(0.022)$ & $15.797(0.022)$ \\
\hline $2005 \mathrm{Jul} 5$ & 3557.07 & 23.41 & $19.524(0.031)$ & $17.766(0.022)$ & $16.369(0.022)$ \\
\hline
\end{tabular}

Notes. Uncertainties are $1 \sigma$.

${ }^{a}$ Relative to the epoch of $B$-band maximum $(\mathrm{JD}=2,453,533.66)$.

\subsection{Hubble Space Telescope UV and NIR Observations}

\subsubsection{ACS UV Photometry}

Imaging of SN 2005cf was carried out with the HST ACS High Resolution Camera (HRC) during Cycle 13. The field of view of this CCD-based instrument is about $29^{\prime \prime} \times 25^{\prime \prime}$ with a pixel scale of $0^{\prime \prime} 028 \times 0^{\prime \prime} 025$. The observations were made in the F220W, F250W, and F330W bands, with exposure times of 1040 s, $800 \mathrm{~s}$, and $360 \mathrm{~s}$, respectively. The exposure time was split into several equal segments that were used in the data-reduction process to reject cosmic-ray events. The data produced by the Space Telescope Science Institute (STScI) reduction pipeline had bias and dark-current frames subtracted and were divided by a flatfield image. In addition, known hot pixels and other defects were masked. Individual exposures were combined using the MultiDrizzle task within STSDAS to reject cosmic rays and perform geometric-distortion corrections.

To get the optimal signal-to-noise ratio $(\mathrm{S} / \mathrm{N})$, we performed aperture photometry in all of the drizzled images using an aperture radius of 4 pixels $\left(\sim 00^{\prime} 1\right)$. The background level was determined from the median counts in an annulus of radius 100-130 pixels. The measured magnitudes were further corrected to an infinite-radius aperture and placed on the Vega magnitude system (Sirianni et al. 2005). The final HST ACS UV magnitudes of SN 2005cf are listed in Table 6. Uncertainties were calculated by combining in quadrature the Poisson photon errors, the readoutnoise errors from the pixels within the aperture, errors in the aperture correction, as well as an error of $2 \%$ from the photometric calibration of ACS/HRC images (Pavlovsky et al. 2004). Note, however, that the uncertainty of the photometric calibration for filter F $220 \mathrm{~W}$ may exceed $2 \%$ because of a red leak.

\subsubsection{NICMOS NIR Photometry}

NIR observations were obtained with HST NICMOS3, which has a scale of $0^{\prime} \cdot 20 \mathrm{pixel}^{-1}$ and a field of view of $51^{\prime \prime} \times 51^{\prime \prime}$. Images were acquired through the F110W and F160W filters (see Figure 5 for the transmission curves) at similar epochs as the optical ones with the ACS. The data were preprocessed using the STSDAS package CALNICA and the latest reference files provided by STScI. Unlike the ACS, the NICMOS calibrated data are given in count rate $\left(\mathrm{DN} \mathrm{s}^{-1}\right.$, where $\mathrm{DN}$ are data-number counts).

Aperture photometry was performed on the calibrated NICMOS3 images. Counts were summed within an aperture of 5.5 pixel radius $(1$ '. 10 , the size of the aperture used for the standardstar measurements) centered on the source. To correct for a nominal infinite aperture, the measured count rates in F110W and F160W were, respectively, multiplied by 1.056 and 1.087 . The total count rates were then converted into flux using the recently determined photometric scale factors, $1.59 \times 10^{-6} \mathrm{Jy} \mathrm{s} \mathrm{DN}^{-1}$ at $1.1 \mu \mathrm{m}$ and $1.93 \times 10^{-6} \mathrm{Jy} \mathrm{s} \mathrm{DN}^{-1}$ at $1.6 \mu \mathrm{m}$. Corresponding zero points were calculated on the Vega system, assuming the zero-magnitude flux densities of 1886 and $1086 \mathrm{Jy}$ and the effective wavelengths of $1.12 \mu \mathrm{m}$ and $1.60 \mu \mathrm{m}$ for F110W and F160W, respectively. All of the above parameters involved in the calculations were taken from the Web site for HST NICMOS photometry. ${ }^{17}$

As the NICMOS3 filters do not match ground-based $J H K$ well (especially the F110W filter, which is much broader and bluer), it is necessary to apply $S$-corrections and color-term corrections to place the photometry on the ground-based $\mathrm{JHK}$ system. A total of six stars (two white dwarfs, two solar analogs, and two red stars) have been observed through both the NICMOS NIR system (F110W, F160W, and F222M filters) and the ground-based $J H K$ system (Persson et al. 1998). These stars, along with the Sun, Vega, and Sirius with model IR spectra from the Kurucz Web site, ${ }^{18}$ allow us to establish an approximate transformation (with an uncertainty of $\sim 0.1 \mathrm{mag}$ ) between the NICMOS3 and the ground-based NIR systems for normal stars. In computing the NIR $S$-corrections we used data for SN 1999ee (Hamuy et al. 2002), which has relatively good temporal coverage of the NIR spectra and a value of $\Delta m_{15}$ similar to that of SN $2005 \mathrm{cf}$.

Table 7 gives the color-, $K$-, and $S$-corrected NIR photometric results from the HST NICMOS3 images, consistent with the ground-based measurements considering the uncertainties from the transformations. The $S$ - and color-corrections that were added to normalize the photometry to the Persson et al. (1998) system are also listed.

\subsection{Swift UVOT Optical/UV Observations}

SN 2005cf was also observed with the Ultraviolet/Optical Telescope (UVOT; Roming et al. 2005) onboard the Swift observatory (Gehrels et al. 2004), covering from 8 days before to 42 days after the $B$-band maximum. The photometric observations

\footnotetext{
17 http://www.stsci.edu/hst/nicmos/performance/photometry/

18 http://kurucz.harvard.edu/
} 
Table 7

HST NICMOS3 NIR Photometry of SN 2005cf

\begin{tabular}{|c|c|c|c|c|c|c|c|c|}
\hline \multirow[t]{2}{*}{ UT Date } & \multirow{2}{*}{$\begin{array}{c}\mathrm{JD} \\
-2,450,000\end{array}$} & \multirow[t]{2}{*}{ Phase $^{\mathrm{a}}$} & \multirow[t]{2}{*}{$J^{\mathrm{b}}$ (mag) } & \multirow[t]{2}{*}{$H^{\mathrm{b}}(\mathrm{mag})$} & \multirow{2}{*}{$\begin{array}{c}\text { Color Correction (mag) } \\
\text { F110W } \rightarrow J\end{array}$} & \multicolumn{3}{|c|}{ S-Correction (mag) } \\
\hline & & & & & & $\mathrm{F} 160 \mathrm{~W} \rightarrow H$ & $\mathrm{~F} 110 \mathrm{~W} \rightarrow J$ & $\mathrm{~F} 160 \mathrm{~W} \rightarrow H$ \\
\hline 2005 Jun 3 & 3525.08 & -8.58 & $14.297(0.030)$ & $14.145(0.025)$ & 0.083 & 0.032 & -0.054 & -0.183 \\
\hline 2005 Jun 7 & 3527.15 & -6.51 & $14.011(0.029)$ & $13.866(0.023)$ & 0.075 & 0.031 & -0.058 & -0.171 \\
\hline 2005 Jun 10 & 3529.81 & -3.85 & $13.880(0.029)$ & $13.853(0.023)$ & 0.145 & 0.044 & -0.067 & -0.163 \\
\hline 2005 Jun 13 & 3533.14 & -0.52 & $13.900(0.029)$ & $13.912(0.023)$ & 0.156 & 0.046 & -0.080 & -0.154 \\
\hline 2005 Jun 16 & 3536.74 & 3.08 & $14.348(0.030)$ & $14.194(0.025)$ & 0.115 & 0.038 & 0.043 & -0.136 \\
\hline 2005 Jun 18 & 3538.87 & 5.21 & $14.469(0.030)$ & $14.249(0.025)$ & 0.120 & 0.039 & 0.120 & -0.133 \\
\hline 2005 Jun 23 & 3544.01 & 10.35 & $15.159(0.032)$ & $14.361(0.027)$ & 0.077 & 0.031 & 0.530 & -0.237 \\
\hline 2005 Jun 27 & 3548.08 & 14.42 & $15.459(0.032)$ & $14.199(0.026)$ & 0.001 & 0.018 & 0.810 & -0.310 \\
\hline 2005 Jul 1 & 3552.08 & 18.42 & $15.432(0.032)$ & $14.158(0.025)$ & -0.050 & 0.008 & 0.810 & -0.250 \\
\hline $2005 \mathrm{Jul} 7$ & 3558.00 & 24.34 & $15.300(0.032)$ & $14.060(0.025)$ & -0.047 & 0.009 & 0.790 & -0.240 \\
\hline
\end{tabular}

Notes. Uncertainties are $1 \sigma$.

${ }^{\text {a }}$ Relative to the epoch of $B$-band maximum (JD $\left.=2,453,533.66\right)$.

${ }^{\mathrm{b}}$ The $J$ - and $H$-band magnitudes were converted, respectively, from the F110W-and F160W-band magnitudes using the color- and $S$-corrections listed in Columns 6-9.

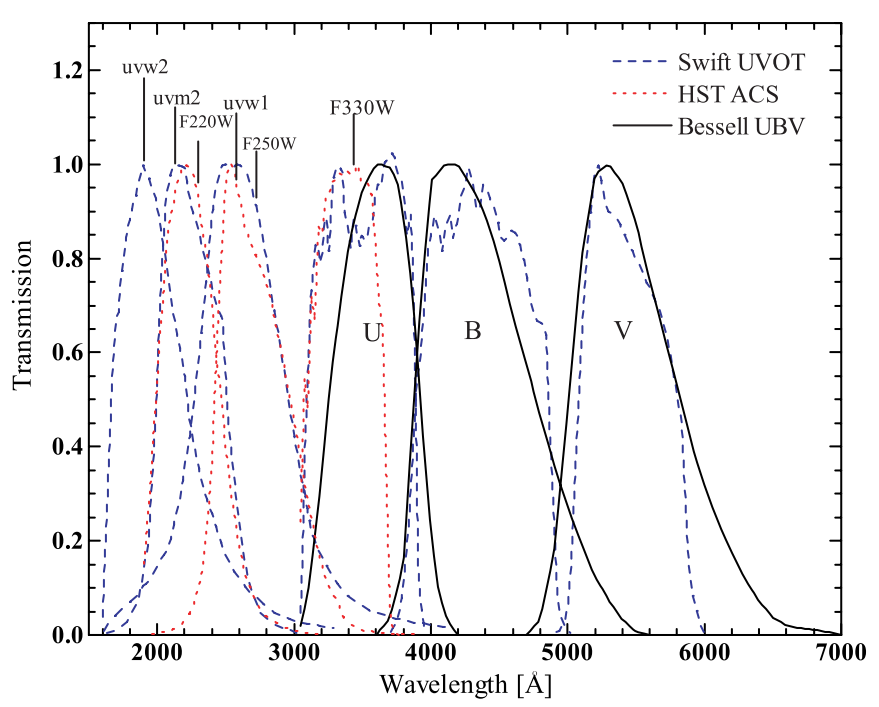

Figure 6. Comparison of transmission curves of Swift UVOT filters with HST ACS in the UV and with the Johnson system in $U B V$.

(A color version of this figure is available in the online journal.)

were performed in three UV filters (uvw1, uvm2, and uvw2) and three broadband optical filters $(U, B$, and $V)$. As shown in Figure 6, the instrumental response curves of the UVOT uvm2 and uvw1 filters are, respectively, very similar to those of the HST ACS F220W and F250W filters. The UVOT optical filters are close to the standard Johnson system in $B$ and $V$ but exhibit noticeable difference in $U$.

Images of SN 2005cf were retrieved from the Swift Data Center, and were reprocessed utilizing an improved plate scale for the uvw2 images and corrections to the exposure times in the image headers (Brown et al. 2009). To maximize the S/N, we performed aperture photometry using an optimal aperture of 3".0 radius suggested by $\mathrm{Li}$ et al. (2006), after first subtracting the host-galaxy light using a template image. Since the UVOT is a photon-counting detector and suffers from coincidence losses when observing bright sources, the observed counts of SN 2005cf were corrected for such losses using the empirical relation described by Poole et al. (2008). Finally, aperture corrections were applied to bring the measurements from an aperture of 3.'0 to the 5."0 aperture with which the photometric zero points are calibrated on the Vega magnitude system.
As the instrumental response curves of the UVOT optical filters do not follow exactly those of the Johnson $U B V$ system (see also Figure 6), some color terms are expected. We calculated the synthetic color terms by integrating the product of the instrumental response of the UVOT in the optical with the spectra of 93 spectrophotometric Landolt (1992) standard stars published by Stritzinger et al. (2005). These are $-0.104,-0.012$, and -0.030 in a linear correlation of the parameter pairs $(U, U-B),(B, B-V)$, and $(V, B-V)$, respectively.

The synthetic color terms are small in the $B$ and $V$ bands but relatively large in the $U$ band. Our determinations are consistent with those measured by Poole et al. (2008) who preferred a polynomial expression. The $S$-corrections of the UVOT optical filters were derived using spectra of SN 2005cf; they are given in Table 8 (Columns 10-12) and are non-negligible in the $U$ band (e.g., 0.1-0.2 mag). Columns 4-9 in Table 8 list the final UVOT UV/optical magnitudes. The magnitudes in optical have been corrected for the color- and $S$-corrections.

\section{LIGHT CURVES OF SN 2005CF}

Figure 7 shows the uvoir light curves of SN 2005cf. The $S$ - and $K$-corrections have been applied to the light curves in all of the optical and NIR bands. No $S$ - or $K$-corrections were applied to the UV data. The optical light curves were sampled nearly daily during the period $t \approx-12$ to +90 days (times given relative to $B$-band maximum), making SN $2005 \mathrm{cf}$ one of the best-observed SNe Ia. The morphology of the light curves resembles that of a normal $\mathrm{SN}$ Ia, having a shoulder in the $R$ band and pronounced secondary-maximum features in the NIR bands. The NIR light curves of SN 2005cf reached their peaks slightly earlier than the $B$-band curve. This is also the case for the UV light curves, which are found to have narrower peaks, with the exceptions of the HST ACS F220W and the Swift UVOT uvm2 filters. Detailed analysis of the light curves is described in the following subsections.

\subsection{Optical Light Curves}

A polynomial fit to the $B$-band light curve around maximum brightness yields $B_{\max }=13.63 \pm 0.02 \mathrm{mag}$ on JD 2,453,533.66 \pm 0.28 (2005 June 12.16). The maximum epoch $t_{\max }(B)$ is consistent with the estimate by P07. The derived value of $t_{\max }(B)$ indicates that our observations started from -11.9 days 
Table 8

Swift UVOT Ultraviolet/Optical Photometry of SN 2005cf

\begin{tabular}{|c|c|c|c|c|c|c|c|c|c|c|c|}
\hline UT Date & JD-2,450,000 & Phase $^{\mathrm{a}}$ & uvw2 (mag) & uvm2 (mag) & uvw1 (mag) & $U$ (mag) & $B$ (mag) & $V(\mathrm{mag})$ & $S c_{U}$ & $S c_{B}$ & $S c_{V}$ \\
\hline 2005 Jun 4 & 3525.55 & -8.11 & $17.79(0.09)$ & $>19.69$ & $16.34(0.07)$ & $14.31(0.05)$ & $14.26(0.05)$ & $14.36(0.05)$ & -0.17 & -0.02 & 0.01 \\
\hline 2005 Jun 5 & 3526.55 & -7.11 & $17.53(0.08)$ & $>20.00$ & $15.91(0.06)$ & $13.99(0.05)$ & $14.17(0.09)$ & $14.16(0.05)$ & -0.13 & -0.01 & 0.01 \\
\hline 2005 Jun 6 & 3527.55 & -6.11 & $17.33(0.08)$ & $19.45(0.31)$ & $15.58(0.06)$ & $13.76(0.05)$ & $13.92(0.06)$ & $14.02(0.04)$ & -0.11 & -0.01 & 0.01 \\
\hline 2005 Jun 8 & 3530.43 & -3.23 & $16.91(0.07)$ & $18.76(0.31)$ & $15.18(0.05)$ & $13.37(0.06)$ & $13.66(0.06)$ & $13.73(0.05)$ & -0.12 & -0.01 & 0.01 \\
\hline 2005 Jun 9 & 3530.77 & -2.89 & $16.94(0.06)$ & $19.26(0.26)$ & $15.10(0.05)$ & $13.40(0.06)$ & $13.65(0.07)$ & $13.70(0.05)$ & -0.12 & -0.01 & 0.01 \\
\hline 2005 Jun 10 & 3531.97 & -1.69 & $16.85(0.06)$ & $18.36(0.16)$ & $15.10(0.05)$ & $13.34(0.06)$ & $13.60(0.07)$ & $13.66(0.05)$ & -0.12 & -0.01 & 0.01 \\
\hline 2005 Jun 11 & 3533.05 & -0.61 & $16.83(0.06)$ & $18.50(0.16)$ & $15.13(0.05)$ & $\ldots$ & $\ldots$ & $13.61(0.06)$ & $\ldots$ & $\ldots$ & 0.01 \\
\hline 2005 Jun 16 & 3538.26 & 4.60 & $17.15(0.07)$ & $18.32(0.15)$ & $15.39(0.05)$ & $\ldots$ & $\ldots$ & $13.58(0.06)$ & $\ldots$ & $\ldots$ & 0.02 \\
\hline 2005 Jun 17 & 3538.74 & 5.08 & $17.07(0.07)$ & $18.31(0.15)$ & $15.44(0.05)$ & $\ldots$ & $\ldots$ & $13.63(0.06)$ & $\ldots$ & $\ldots$ & 0.02 \\
\hline 2005 Jun 20 & 3542.15 & 8.49 & $17.39(0.07)$ & $18.47(0.15)$ & $15.83(0.06)$ & $\ldots$ & $\ldots$ & $13.70(0.06)$ & $\ldots$ & $\ldots$ & 0.03 \\
\hline 2005 Jun 22 & 3543.70 & 10.04 & $17.47(0.08)$ & $18.58(0.20)$ & $16.03(0.09)$ & $\ldots$ & $\ldots$ & $13.75(0.07)$ & $\ldots$ & $\ldots$ & 0.03 \\
\hline 2005 Jun 26 & 3548.26 & 14.60 & $18.06(0.10)$ & $18.62(0.18)$ & $16.63(0.07)$ & $14.82(0.05)$ & $14.65(0.05)$ & $14.09(0.05)$ & -0.21 & 0 & 0.04 \\
\hline 2005 Jun 29 & 3550.51 & 16.85 & 18.21(0.11) & 18.92(0.24) & $16.96(0.09)$ & $15.14(0.05)$ & $14.91(0.05)$ & $14.25(0.05)$ & -0.22 & 0 & 0.04 \\
\hline 2005 Jul 12 & 3564.32 & 30.66 & $19.36(0.29)$ & $>19.76$ & $18.17(0.16)$ & $16.46(0.08)$ & $16.07(0.06)$ & $14.94(0.05)$ & -0.17 & 0 & 0.06 \\
\hline 2005 Jul 23 & 3575.16 & 41.50 & $\cdots$ & $\ldots$ & $\cdots$ & $17.05(0.06)$ & $16.65(0.05)$ & $15.60(0.05)$ & -0.18 & 0 & 0.05 \\
\hline 2005 Jul 24 & 3575.97 & 42.31 & 19.77(0.19) & $>20.29$ & $18.60(0.11)$ & $\cdots$ & $\cdots$ & $\cdots$ & $\cdots$ & $\cdots$ & $\cdots$ \\
\hline
\end{tabular}

Notes. Uncertainties are $1 \sigma$.

${ }^{\text {a }}$ Relative to the epoch of $B$-band maximum $(\mathrm{JD}=2,453,533.66)$.

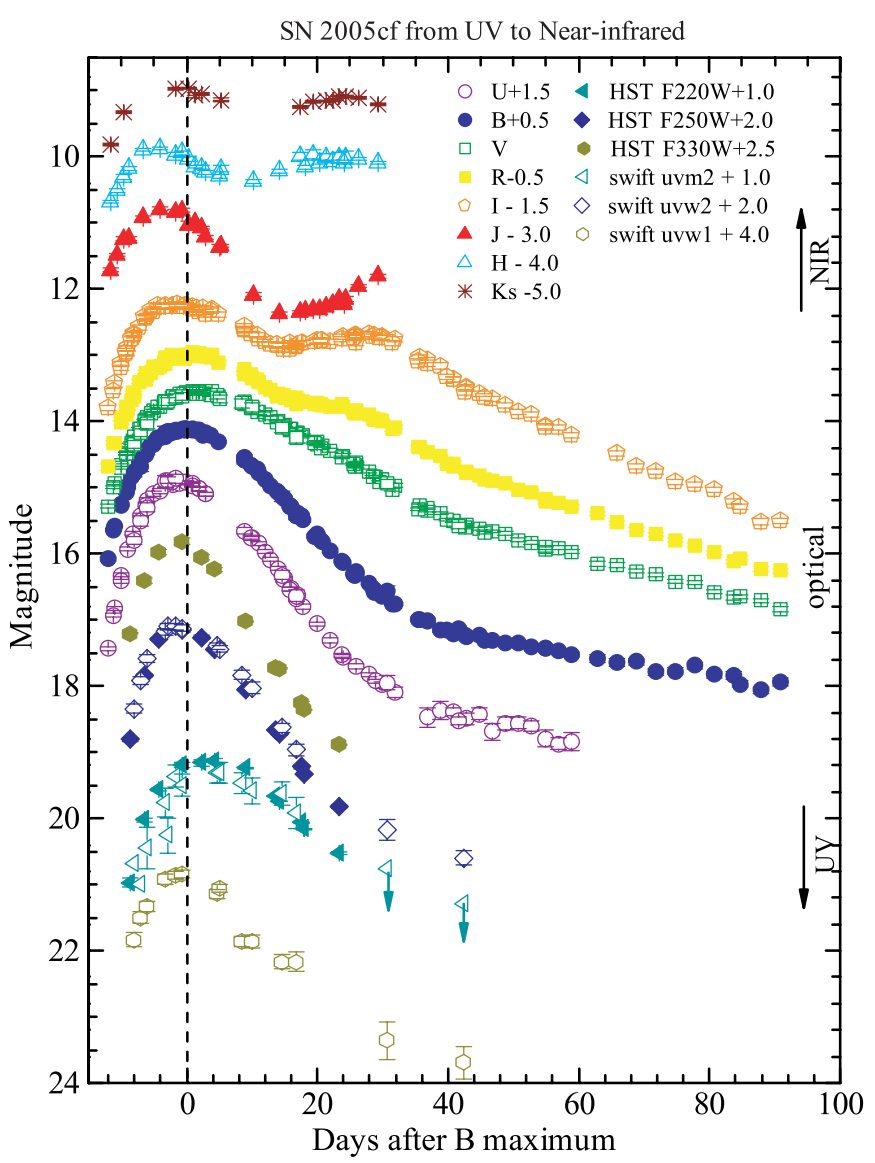

Figure 7. UV, optical, and NIR light curves of SN 2005cf. The UV light curves were obtained from the photometry with HST ACS and the Swift UVOT, the $U B V R I$ optical photometry was collected at eight ground-based telescopes as well as the $S$ wift UVOT, and the $J H K_{s}$ data were taken with the $1.3 \mathrm{~m}$ PAIRITEL and with HST NICMOS3.

(A color version of this figure is available in the online journal.)

and extended to +91.0 days with respect to the $B$ maximum. Likewise, the $V$-band light curve reached a peak magnitude of $13.56 \pm 0.02$ on JD 2,453,535.54 \pm 0.33 , about 1.9 days after $t_{\max }(B)$. The fitting parameters for the maxima in the other bands
Table 9

Light-Curve Parameters of SN 2005cf

\begin{tabular}{lcccc}
\hline \hline Band & $\begin{array}{c}\lambda_{\text {central }} \\
(\AA)\end{array}$ & $\begin{array}{c}t_{\max } \\
-2,450,000\end{array}$ & $\begin{array}{c}m_{\text {peak }} \\
(\mathrm{mag})\end{array}$ & $\begin{array}{c}\Delta m_{15}^{\mathrm{a}} \\
(\mathrm{mag})\end{array}$ \\
\hline uvw2 & 1928 & $3533.05 \pm 0.50$ & $16.84 \pm 0.05$ & $1.11 \pm 0.06$ \\
uvm2 & 2246 & $3537.86 \pm 0.71$ & $18.30 \pm 0.13$ & $0.75 \pm 0.08$ \\
uvw1 & 2600 & $3532.35 \pm 0.44$ & $15.10 \pm 0.04$ & $1.44 \pm 0.05$ \\
F220W & 2228 & $3537.17 \pm 0.48$ & $18.14 \pm 0.05$ & $1.00 \pm 0.06$ \\
F250W & 2696 & $3532.49 \pm 0.42$ & $15.13 \pm 0.04$ & $1.54 \pm 0.05$ \\
F330W & 3354 & $3532.30 \pm 0.40$ & $13.31 \pm 0.04$ & $1.91 \pm 0.05$ \\
$U$ & 3650 & $3532.42 \pm 0.30$ & $13.37 \pm 0.03$ & $1.26 \pm 0.04$ \\
$B$ & 4450 & $3533.66 \pm 0.28$ & $13.63 \pm 0.02$ & $1.05 \pm 0.03$ \\
$V$ & 5500 & $3535.54 \pm 0.33$ & $13.56 \pm 0.02$ & $0.62 \pm 0.03$ \\
$R$ & 6450 & $3534.80 \pm 0.26$ & $13.53 \pm 0.03$ & $0.67 \pm 0.03$ \\
$I$ & 7870 & $3532.56 \pm 0.34$ & $13.76 \pm 0.04$ & $0.59 \pm 0.03$ \\
$J$ & 12700 & $3530.54 \pm 0.44$ & $13.78 \pm 0.05$ & $1.45 \pm 0.05$ \\
$H$ & 16700 & $3529.48 \pm 0.42$ & $13.84 \pm 0.04$ & $0.42 \pm 0.05$ \\
$K$ & 22200 & $3530.32 \pm 0.59$ & $13.94 \pm 0.05$ & $0.40 \pm 0.06$ \\
\hline
\end{tabular}

Notes. Uncertainties are $1 \sigma$.

${ }^{\text {a }}$ The magnitude decline in 15 days after the initial maximum of the light curve.

are presented in Table 9, together with the initial decline rates within the first 15 days after their maxima (see below).

From the $B$ - and $V$-band light curves, we derive an observed $\Delta m_{15}(B)=1.05 \pm 0.03 \mathrm{mag}$ and $B_{\max }-V_{\max }=0.07 \pm 0.03$ mag. ${ }^{19}$ These values are slightly smaller and redder than those given by $\mathrm{P} 07\left[\Delta m_{15}=1.11 \pm 0.05\right.$ and $B_{\max }-V_{\max }=$ $0.01 \pm 0.03]$. We also measured the $B-V$ color at 12 days after the $B$ maximum (Wang et al. 2005), obtaining $0.47 \pm 0.04$ mag. After removal of the Galactic reddening, these color indexes are slightly redder than the intrinsic value (see also descriptions in Section 3.3), suggesting some line-of-sight reddening toward SN 2005cf.

Based on the extremely well sampled photometry in the optical, we constructed the light-curve templates of SN 2005cf by using a smoothing spline function. To obtain better sampling in the $U$ band, the late-phase data from P07 were also included in the fit. We tabulate the template light curves in Table 10,

\footnotetext{
19 The true $\Delta m_{15}(B)$ for SN $2005 \mathrm{cf}$ is $1.07 \pm 0.03$ mag, taking into account
} the reddening effect on the light-curve shape (Phillips et al. 1999). 


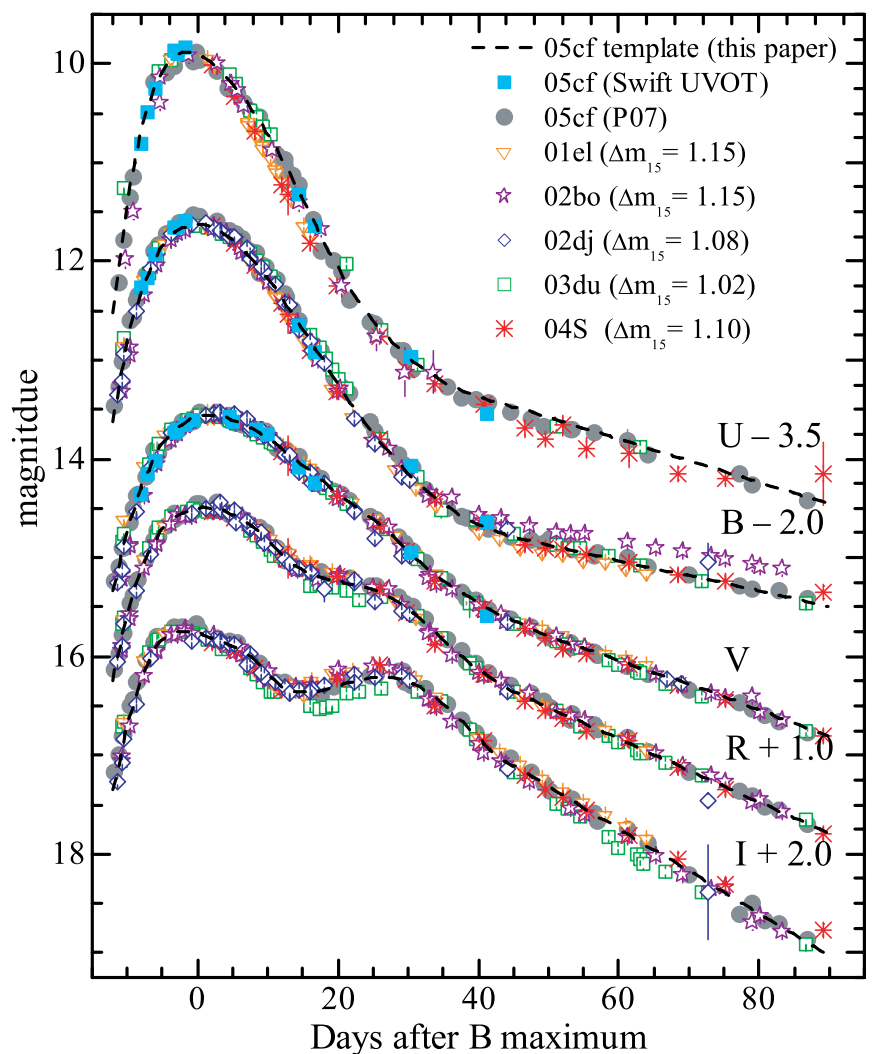

Figure 8. Comparison of $U B V R I$ light curves of SN 2005cf with those published by Pastorello et al. (2007a, 2007b) and with other well-observed SNe Ia: SNe 2001el, 2002bo, 2002dj, 2003du, and 2004S. See text for references.

(A color version of this figure is available in the online journal.)

which are found to be consistent with the published $U B V$ template light curves used in the stretch fitting (Goldhaber et al. 2001; Knop et al. 2003). In Figure 8, we compare the best-fit $U B V R I$ light-curve templates of SN 2005cf with observations from the Swift UVOT and P07. We also compare them with other well observed, nearby SNe Ia having similar $\Delta m_{15}$ values, including $\mathrm{SNe} 2001 \mathrm{el}\left(\Delta m_{15}=1.15 \mathrm{mag}\right.$; Krisciunas et al. 2003), 2002bo $\left(\Delta m_{15}=1.15\right.$ mag; Benetti et al. 2004; Krisciunas et al. 2004a), 2002dj ( $\Delta m_{15}=1.08 \mathrm{mag}$; Pignata et al. 2008), 2003du $\left(\Delta m_{15}=1.02 \mathrm{mag}\right.$; Stanishev et al. 2007), and $2004 \mathrm{~S}$ ( $\Delta m_{15}=1.10 \mathrm{mag}$; Krisciunas et al. 2007). These six objects include all SNe Ia with $1.00<$ $\Delta m_{15}<1.15$ and at least 15 epochs per band of published $U B V R I J H K$ data. The BVRI data for SNe 2002bo, 2002dj, and 2004S obtained with KAIT (M. Ganeshalingam et al. 2009, in preparation) are overplotted. The photometric data for the above comparison $\mathrm{SNe}$ Ia were $S$-corrected. To be consistent with SN $2005 \mathrm{cf}$, the $K$-corrections computed with the spectra of the comparison $\mathrm{SNe}$ and/or the spectra of SN 2005cf were also applied to their photometry. All of the light curves of the comparison $\mathrm{SNe}$ Ia have been normalized to the epoch of their $U B V R I$ maxima and shifted in their peaks to match the templates of SN 2005cf.

The overall comparison with the photometry of P07 reveals some systematic differences, especially at the earlier phases, as shown in Figure 9. Polynomial fits to their light curves (after applying $K$-corrections) around the maximum give the peak magnitudes in $U B V R I$ as $13.40 \pm 0.06 \mathrm{mag}, 13.57 \pm 0.03 \mathrm{mag}$,
Table 10 U B V RI Template Light Curves of SN 2005 $\mathrm{cf}^{\mathrm{a}}$

\begin{tabular}{|c|c|c|c|c|c|}
\hline Days & $U$ & $B$ & $V$ & $R$ & $I$ \\
\hline-12 & 2.58 & 2.00 & 1.78 & 1.75 & 1.61 \\
\hline-11 & 1.97 & 1.55 & 1.45 & 1.37 & 1.28 \\
\hline-10 & 1.48 & 1.20 & 1.17 & 1.05 & 0.97 \\
\hline-9 & 1.08 & 0.91 & 0.93 & 0.80 & 0.70 \\
\hline-8 & 0.76 & 0.68 & 0.72 & 0.60 & 0.48 \\
\hline-7 & 0.51 & 0.49 & 0.56 & 0.44 & 0.31 \\
\hline-6 & 0.33 & 0.35 & 0.41 & 0.32 & 0.18 \\
\hline-5 & 0.19 & 0.23 & 0.30 & 0.22 & 0.09 \\
\hline-4 & 0.09 & 0.14 & 0.21 & 0.14 & 0.04 \\
\hline-3 & 0.04 & 0.08 & 0.14 & 0.09 & 0.01 \\
\hline-2 & 0.01 & 0.03 & 0.08 & 0.05 & 0 \\
\hline-1 & 0 & 0.01 & 0.04 & 0.02 & 0.01 \\
\hline 0 & 0.02 & 0 & 0.02 & 0.01 & 0.02 \\
\hline 1 & 0.05 & 0.01 & 0 & 0 & 0.04 \\
\hline 2 & 0.10 & 0.03 & 0 & 0 & 0.05 \\
\hline 3 & 0.16 & 0.06 & 0.01 & 0.02 & 0.08 \\
\hline 4 & 0.22 & 0.11 & 0.02 & 0.04 & 0.10 \\
\hline 5 & 0.30 & 0.16 & 0.04 & 0.07 & 0.13 \\
\hline 6 & 0.39 & 0.23 & 0.07 & 0.11 & 0.16 \\
\hline 7 & 0.48 & 0.30 & 0.11 & 0.16 & 0.21 \\
\hline 8 & 0.58 & 0.37 & 0.15 & 0.21 & 0.27 \\
\hline 9 & 0.69 & 0.46 & 0.19 & 0.27 & 0.33 \\
\hline 10 & 0.80 & 0.55 & 0.24 & 0.34 & 0.41 \\
\hline 11 & 0.92 & 0.64 & 0.29 & 0.40 & 0.48 \\
\hline 12 & 1.04 & 0.73 & 0.34 & 0.47 & 0.54 \\
\hline 13 & 1.17 & 0.84 & 0.39 & 0.53 & 0.57 \\
\hline 14 & 1.31 & 0.94 & 0.45 & 0.59 & 0.59 \\
\hline 15 & 1.45 & 1.05 & 0.50 & 0.63 & 0.59 \\
\hline 16 & 1.59 & 1.15 & 0.56 & 0.66 & 0.59 \\
\hline 17 & 1.74 & 1.27 & 0.61 & 0.68 & 0.59 \\
\hline 18 & 1.89 & 1.38 & 0.67 & 0.70 & 0.57 \\
\hline 19 & 2.02 & 1.49 & 0.72 & 0.72 & 0.56 \\
\hline 20 & 2.15 & 1.60 & 0.78 & 0.74 & 0.54 \\
\hline 21 & 2.27 & 1.71 & 0.83 & 0.75 & 0.52 \\
\hline 22 & 2.38 & 1.81 & 0.88 & 0.76 & 0.50 \\
\hline 23 & 2.49 & 1.91 & 0.93 & 0.78 & 0.48 \\
\hline 24 & 2.59 & 2.00 & 0.98 & 0.80 & 0.47 \\
\hline 25 & 2.68 & 2.10 & 1.04 & 0.82 & 0.46 \\
\hline 26 & 2.77 & 2.19 & 1.09 & 0.85 & 0.45 \\
\hline 27 & 2.85 & 2.27 & 1.14 & 0.89 & 0.45 \\
\hline 28 & 2.92 & 2.36 & 1.21 & 0.93 & 0.46 \\
\hline 29 & 2.99 & 2.44 & 1.29 & 0.97 & 0.47 \\
\hline 30 & 3.06 & 2.51 & 1.35 & 1.02 & 0.50 \\
\hline 31 & 3.12 & 2.58 & 1.42 & 1.08 & 0.53 \\
\hline 34 & 3.27 & 2.77 & 1.60 & 1.27 & 0.66 \\
\hline 37 & 3.38 & 2.92 & 1.75 & 1.47 & 0.86 \\
\hline 40 & 3.46 & 3.03 & 1.88 & 1.64 & 1.08 \\
\hline 43 & 3.53 & 3.11 & 1.99 & 1.77 & 1.28 \\
\hline 46 & 3.58 & 3.16 & 2.09 & 1.89 & 1.40 \\
\hline 49 & 3.65 & 3.21 & 2.18 & 1.98 & 1.53 \\
\hline 52 & 3.72 & 3.26 & 2.26 & 2.08 & 1.66 \\
\hline 57 & 3.83 & 3.34 & 2.39 & 2.23 & 1.88 \\
\hline 62 & 3.94 & 3.42 & 2.51 & 2.39 & 2.10 \\
\hline 65 & 4.00 & 3.46 & 2.58 & 2.49 & 2.23 \\
\hline 70 & 4.11 & 3.54 & 2.71 & 2.64 & 2.45 \\
\hline 75 & 4.22 & 3.62 & 2.84 & 2.80 & 2.67 \\
\hline 80 & 4.33 & 3.70 & 2.98 & 2.96 & 2.89 \\
\hline 85 & 4.44 & 3.78 & 3.12 & 3.12 & 3.10 \\
\hline 90 & 4.55 & 3.86 & 3.25 & 3.28 & 3.32 \\
\hline
\end{tabular}

Note.

a All of the light curves have been normalized to the $B$-band maximum epoch and their peak values listed in Table 9. All units are mag. 
Table 11

Intrinsic Color vs. $\Delta m_{15}(B)$ Relation

\begin{tabular}{lccccc}
\hline \hline Color & $a$ & $b_{1}$ & $b_{2}$ & $b_{3}$ & $\sigma$ \\
\hline$B_{\max }-V_{\max }{ }^{\mathrm{a}}$ & $-0.09(0.04)$ & $0.15(0.03)$ & $\ldots$ & $\ldots$ & 0.03 \\
$B_{\max }-V_{\max }{ }^{\mathrm{b}}$ & $-1.61(0.35)$ & $2.60(0.47)$ & $\ldots$ & $\ldots$ & 0.11 \\
$V_{\max }-I_{\max }{ }^{\mathrm{a}}$ & $-0.27(0.01)$ & $0.22(0.03)$ & $\ldots$ & $\ldots$ & 0.06 \\
$V_{\max }-I_{\max }{ }^{\mathrm{b}}$ & $-1.03(0.10)$ & $1.45(0.14)$ & $\ldots$ & $\ldots$ & 0.06 \\
$(B-V)_{12}$ & $0.25(0.01)$ & $0.34(0.05)$ & $-0.38(0.21)$ & $1.98(0.23)$ & 0.04 \\
$(B-V)_{35}{ }^{\mathrm{a}}$ & $1.02(0.01)$ & $\ldots$ & $\ldots$ & $\ldots$ & 0.04 \\
$(B-V)_{35}{ }^{\mathrm{b}}$ & $0.72(0.07)$ & $0.46(0.10)$ & $\ldots$ & $\ldots$ & 0.04 \\
\hline
\end{tabular}

Notes. Uncertainties are $1 \sigma$

* Color $=a+b_{1}\left(\Delta m_{15}-1.1\right)+b_{2}\left(\Delta m_{15}-1.1\right)^{2}+b_{3}\left(\Delta m_{15}-1.1\right)^{3}$.

a The correlation holds for SNe Ia with $0.8<\Delta m_{15}<1.7$.

b The correlation applies to SNe Ia with $\Delta m_{15} \gtrsim 1.7$.

$13.55 \pm 0.02,13.48 \pm 0.03$, and $13.72 \pm 0.03$, respectively. The $B R I$ peak magnitudes were found to be brighter than ours by about 0.05 mag (cf. Table 9). This may be primarily due to the calibration differences of the standard stars (at least in $B$ and $R$ ), which is also demonstrated by the average difference of the photometry over time between P07 and this paper (e.g., -0.042 \pm 0.004 in $B,-0.027 \pm 0.004 \mathrm{mag}$ in $R$, and $-0.042 \pm 0.005$ in $I$; see the filled circles in Figure 9). Note that the dependence of the difference in $I$ (and perhaps $U$ ) on the SN phase at early epochs may suggest that the instrumental response for either of these two measurements is still not completely removed. It is puzzling, however, that a remarkable difference of $\sim 0.12 \mathrm{mag}$ in the $U$ band for the comparison stars (see Section 2.1.1) is not also seen in the $U$-band SN photometry. Additional, uncorrected systematic effects are probably present.

With the constructed light-curve templates of SN 2005cf, one may also examine whether the $S$ wift UVOT optical observations are consistent with the ground-based data. The mean values of the computed residuals between these two measurements (see the filled square symbols in Figure 9) are $0.027 \pm 0.022$ mag in $U,-0.028 \pm 0.016 \mathrm{mag}$ in $B$, and $0.025 \pm 0.010 \mathrm{mag}$ in $V$, which are comparable to the photometric errors. This demonstrates the great improvements recently achieved in Swift UVOT calibrations (Li et al. 2006; Poole et al. 2008).

Although the light curves of the comparison SNe Ia are similar to each other near maximum brightness, they diverge at earlier (rising) phases. SNe 2001el and 2003du displayed a slower rise rate in each of the $U B V R I$ bands. In contrast, the brightness of SNe 2002bo and 2002dj rose at a faster pace than that of SN 2005cf (see also Pignata et al. 2008). This indicates that the rise time might slightly vary among SNe Ia having similar $\Delta m_{15}$ values, though a larger sample having early-epoch observations is needed to verify this trend.

Differences in the light curves also emerge at late phases, especially in the $B$ band. We measured a late-time decline rate $\beta=1.62 \pm 0.05 \mathrm{mag}$ (100 days) $)^{-1}$ in $B$ for SN 2005cf during the interval $t=40-90$ days, which is comparable to that of SNe 2001el, 2003du, and 2004S at similar phase. While SNe 2002bo and $2002 \mathrm{dj}$ showed much slower decay rates, $\beta \approx 1.2 \mathrm{mag}$ $(100 \text { days })^{-1}$, they happened to be events with high expansion velocity of the photosphere. These fast-expanding SNe may generally have flatter photometric evolution in $B$ at late times, similar to that of SN 2006X (Wang et al. 2008a). ${ }^{20}$

\footnotetext{
20 Measurements of the late-time decline rate in the $U$ band are usually difficult due to the lack of reliable photometry at this phase. High-quality data are needed to test whether the $U$-band data follow the same trend as shown in $B$.
}

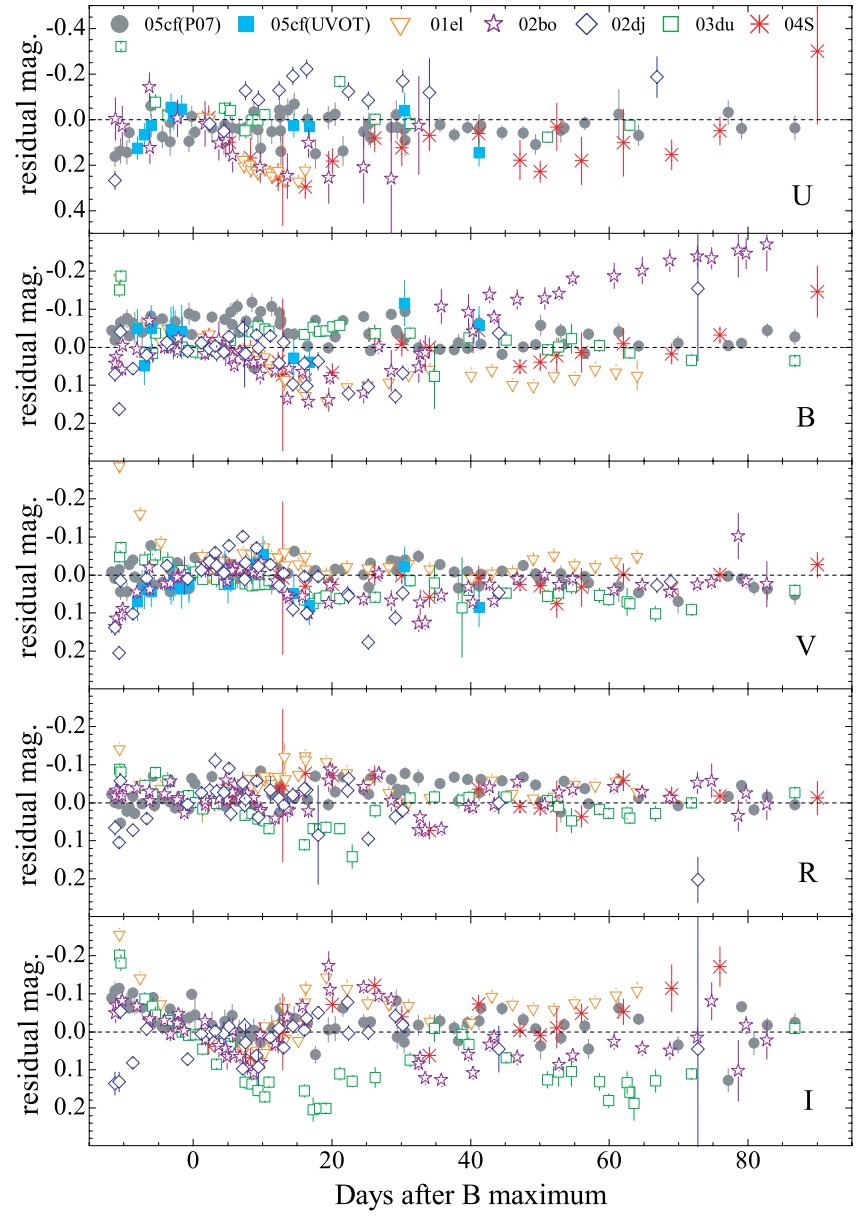

Figure 9. Residual magnitudes of the comparison SNe Ia with respect to the light-curve templates derived from our observations of SN 2005cf.

(A color version of this figure is available in the online journal.)

In contrast to the $V$ - and $R$-band evolution, the light curves in the $U$ and $I$ bands appear more heterogeneous. The largest dispersion is in the premaximum $U$-band evolution and in the I-band secondary-maximum phase. Systematic effects due to filter mismatches might not be fully removed by the $S$-corrections because of the incomplete wavelength and/or temporal coverage for the SN spectra. Nevertheless, part of the dispersion is likely to be intrinsic. Significant spectral variations at broadband $U$ wavelengths were also found in SNe Ia at $z \approx 0.5$ (Ellis et al. 2008). The $U$-band brightness has also been proposed to depend sensitively on the metallicity of the progenitor (e.g., Höflich et al. 1998; Lentz et al. 2000; Sauer et al. 2008), which may vary from one $\mathrm{SN}$ to another, while emission in the $I$ band is heavily affected by the Ca II NIR triplet absorption, which is found to vary substantially among SNe Ia at early phases (see Figure 18 in Section 4.1).

\subsection{The UV Light Curves}

UV observations are important for understanding SN Ia physics as well as possible differences among the progenitors in a range of environments. Due to the requirement of space-based observations, however, UV data for SNe Ia have been sparse; see Panagia et al. (2003) and Foley et al. (2008) for summaries of International Ultraviolet Explorer (IUE) and HST spectra. In HST Cycle 13, extensive UV observations of SNe Ia were conducted (program GO-10182; PI Filippenko); unfortunately, 


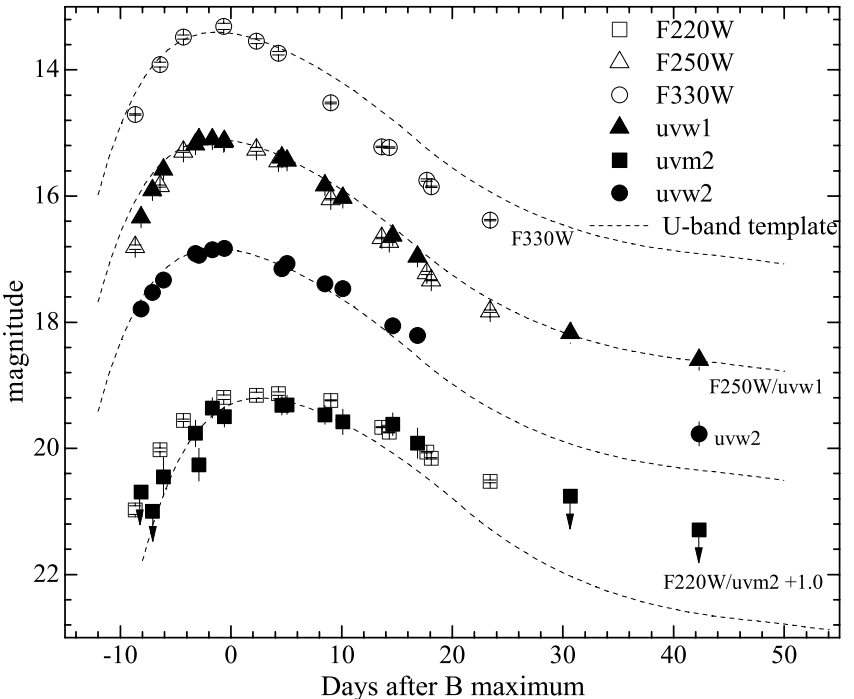

Figure 10. UV light curves of SN 2005cf, obtained with the HST ACS (F220W, F250W, and F330W) and the Swift UVOT (uvw1, uvm2, uvw2). The first two and the last two data points in the uvm 2 band are $3 \sigma$ upper limits of the detection. Overlaid is the $U$-band template of SN 2005cf (dashed line).

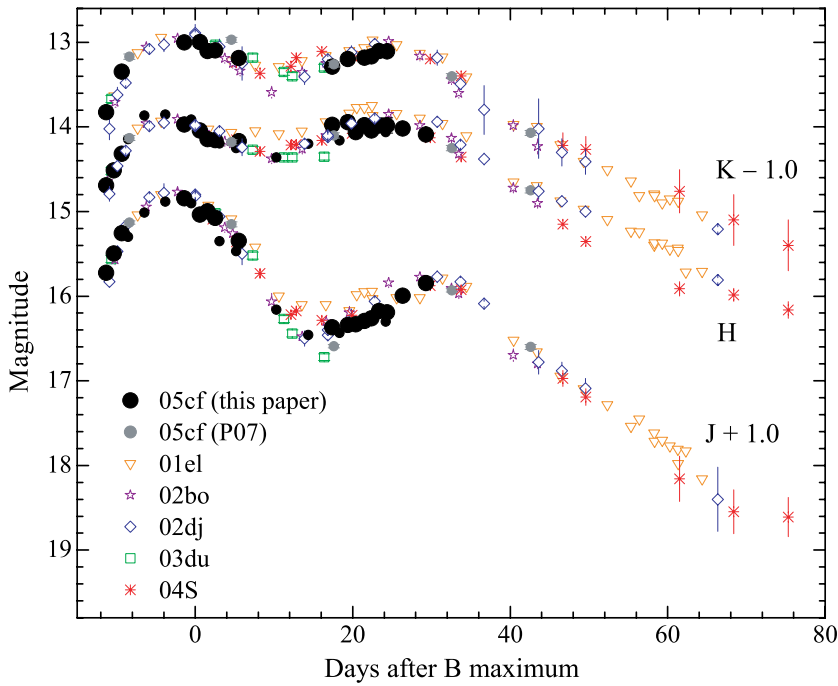

Figure 11. Comparison of the NIR light curves of SN 2005cf with those of SNe 2001el, 2002bo, 2002dj, 2003du, and 2004S. All light curves are shifted in time and magnitude to fit the peak values of SN 2005cf. The data sources are cited in the text.

(A color version of this figure is available in the online journal.)

the Space Telescope Imaging Spectrometer (STIS) failed just before the program began, so the UV prism in ACS was used instead, yielding spectra far inferior to those anticipated with STIS. Recently, Swift has obtained UV photometry of SNe Ia (Brown et al. 2009; P. Milne et al. 2009, in preparation), as well as some low-quality UV grism spectra (F. Bufano et al. 2009, in preparation).

Figure 10 shows the UV light curves of SN 2005cf obtained with the Swift UVOT and the uvw1, uvw2, and uvm2 filters, as well as with the HST ACS and the F220W, F250W, and F330W filters. These two UV data sets span the periods from $t=-8.1$ days to $t=41.8$ days and from $t=-8.6$ days to $t=23.4$ days, respectively. Overlaid are the $U$-band templates of SN 2005cf, shifted to match the peak of the UV bands. With respect to $U$, the F330W-band light curve has a noticeably narrower peak. The UVOT uvw1 light curve closely resembles

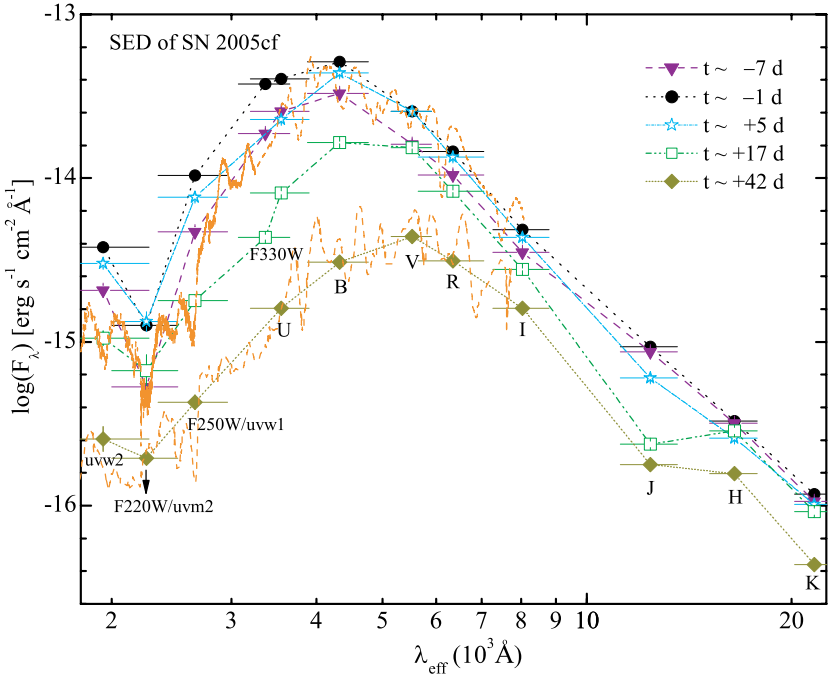

Figure 12. SED evolution of SN 2005cf at $t=-7,-1,+5,+18$, and +42 days after the $B$-band maximum. The photometric points are shown with error bars (vertical ones for uncertainties and horizontal ones for the full width at half-maximum intensity of the filters). Overlaid are the HST FOS spectra of SN 1992A (dotted curves) from Kirshner et al. (1993), obtained at $t \approx+5$ days and +45 days, respectively. Note that the error bar of the flux is in most cases smaller than the size of the symbol.

(A color version of this figure is available in the online journal.)

that in the HST F250W band, with the former being slightly brighter by $-0.09 \pm 0.02 \mathrm{mag}$. Despite the similarity of the filter responses, the UVOT uvm2 light curve appears to be systematically dimmer than that of $H S T$ F220W by $\sim 0.2$ mag at comparable phases. This is perhaps due to the calibration uncertainty or reduction errors, but more data are obviously needed to clarify this difference.

The temporal behavior of the UV photometry of SN 2005cf is similar to the optical behavior, but with different epochs for the maximum brightness. As in the $U$ band, most of the UV light curves reached their maximum slightly before the optical, with the exception of the uvm2/F220W-band photometry, which peaks $\sim 3-4$ days after the $B$ maximum. Another interesting feature is that the light-curve peak in these two filters appears to be much fainter and broader than that of the other UV filters on both sides. A similar feature was seen in SN 2005am (Brown et al. 2005), a normal SN Ia resembling SN 1992A (Kirshner et al. 1993). Brown et al. (2005) suggested that the faintness in uvm 2 could be explained in terms of a bump of the extinction curve near $2200 \AA$ (Cardelli et al. 1989). Assuming a total reddening of $E(B-V) \approx 0.2$ mag (see Section 3.6), however, we find that the change of $R_{V}$ could only result in a larger extinction in uvm $2 / \mathrm{F} 220 \mathrm{~W}$ by $\sim 0.5 \mathrm{mag}$. This is far below the flux drop of $\sim 1.5 \mathrm{mag}$ with respect to the neighboring bands, as seen in SN 2005cf. The red leak in the UV filters might cause the abnormal behavior in the UV light curves by including some optical light, due to the red tail. However, the report from the most recent check of the HST ACS CCDs shows that such an effect is small in the HST UV filters. ${ }^{21}$ Moreover, the off-band contamination in the F220W filter is found to be larger than that in F250W. This shows that the UV leak may not be the main reason responsible for the faint brightness in the F220W or uvm2 filters.

As in the optical, the light-curve parameters in the UV were obtained by using a polynomial fit to the observations (Table 9).

\footnotetext{
21 http://www.stsci.edu/hst/acs/documents/handbooks/cycle17
} 

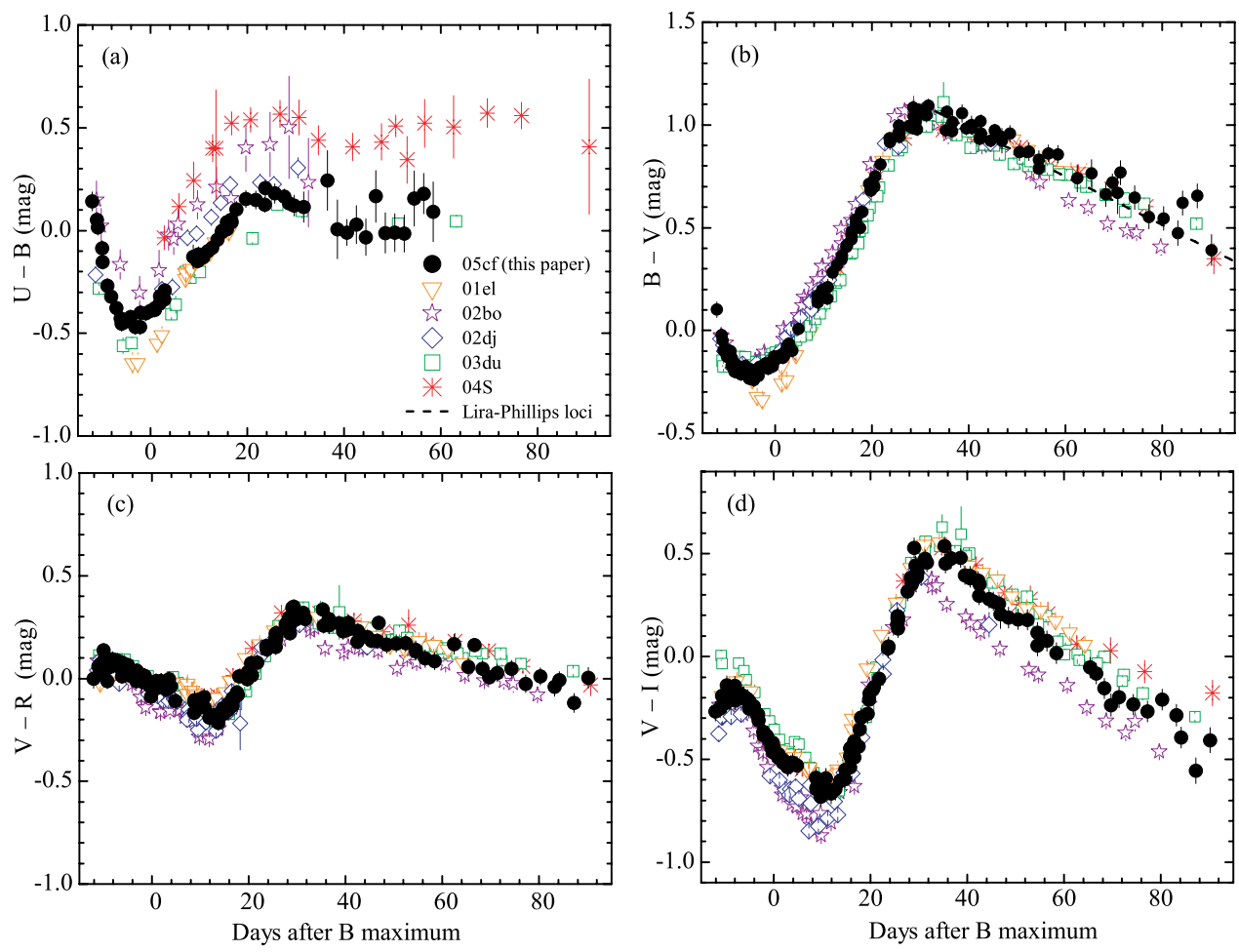

Figure 13. $U-B, B-V, V-R$, and $V-I$ color curves of SN 2005cf compared with those of SNe 2001el, 2002bo, 2002dj, 2003du, and 2004S. All of the comparison SNe have been dereddened. The dash-dotted line in the $B-V$ panel shows the unreddened Lira-Phillips loci. The data sources are cited in the text.

(A color version of this figure is available in the online journal.)

One can see that the luminosity in the F330W band is the brightest, but it declines at the fastest pace after the initial peak. The uvm2-band luminosity is the faintest, and has a postmaximum decay rate much slower than the other UV filters.

\subsection{The NIR Light Curves}

The NIR photometry of SN 2005cf was obtained with PAIRITEL (Wood-Vasey et al. 2008) and HST NICMOS3, spanning from -11.4 days to +29.1 days with respect to $t_{\max }(B)$. Due to significant differences between these two photometric systems, we applied both color- and $S$-corrections to the HST photometry to normalize it to the PAIRITEL system. As shown in Figure 11, the corrected F110W- and F160Wband magnitudes (smaller filled circles) are consistent with the ground-based results.

In Figure 11, the NIR data of the comparison SNe Ia are overlaid. Although the light curves of all these $\mathrm{SNe}$ are similar near maximum, they diverge after the peak phase. As in the optical, $\mathrm{SNe} 2002 \mathrm{dj}$ and 2002bo rise at a faster pace than SNe 2005cf and 2003du in the NIR. Scatter is also present near the secondary-maximum phase; the fast-expanding events tend to exhibit more prominent peaks. The secondary-peak feature in the $J$ band appears somewhat less pronounced in SN 2001el.

The peak magnitudes in the NIR, estimated by fitting the data with the Krisciunas et al. (2004a) templates, are reported in Table 9. In contrast with the rapid decline in $J$ after the primary maximum, the $H$ - and $K$-band light curves show much slower decay at comparable phases.

\subsection{The Spectral Energy Distribution Evolution}

The evolution of the spectral energy distribution (SED) can be best studied through spectroscopy; however, only the optical spectra of SN 2005cf were involved in our study. As an alternative, a rough SED can be constructed from the observed fluxes in various passbands at the same or similar epochs. Since we have photometry in the UV, optical, and NIR bands, covering the 1600-24000 ̊̊ region, we can study the SED evolution of SN 2005cf by means of photometry. Because of the numerous instruments involved in the observations of SN $2005 \mathrm{cf}$, it was not always possible to observe all bands at exactly the same time, and our definition of the "same epoch" refers to a reference date \pm 1 day. The observed apparent magnitudes in each passband were converted to fluxes using the reddening derived in Section 3.6.

Figure 12 shows SEDs at five selected epochs, $t \approx-7$, $-1,+5,+17$, and +42 days after the $B$-band maximum. The SN 2005cf SED went through dramatic changes in going from epochs near $B$ maximum to the nebular phase. The SEDs at $t \approx-7,-1$, and +5 days are very similar, peaking in $B$ but showing a flux deficit in F220W/uvm2 $(\sim 2000-2500 \AA)$. At these early phases, the emission from the $\mathrm{SN}$ dominated in the blue. By $t \approx 17$ days the SED showed a significant deficit at short wavelengths compared with earlier epochs, whereas the NIR remained fairly constant (with the exception of the $J$ band). The flux peak shifted to the $V$ band by $t \approx 42$ days, and the deficit in F220W/uvm2 might become less noticeable, though the measurement from the Swift UVOT is around the detection limit.

To better understand the evolution of the 2000-2500 A region, we overplot the HST FOS spectra of SN 1992A (Kirshner et al. 1993) in Figure 12. One sees that the F220W/uvm2-band flux deficit in SN 2005cf at $t \approx 5$ days is consistent with the strong absorption trough present in the UV spectrum of SN 1992A at similar epochs, although the UV brightness of the latter seems relatively fainter with respect to their optical emission. This absorption feature could arise from multiplets of the iron-peak 


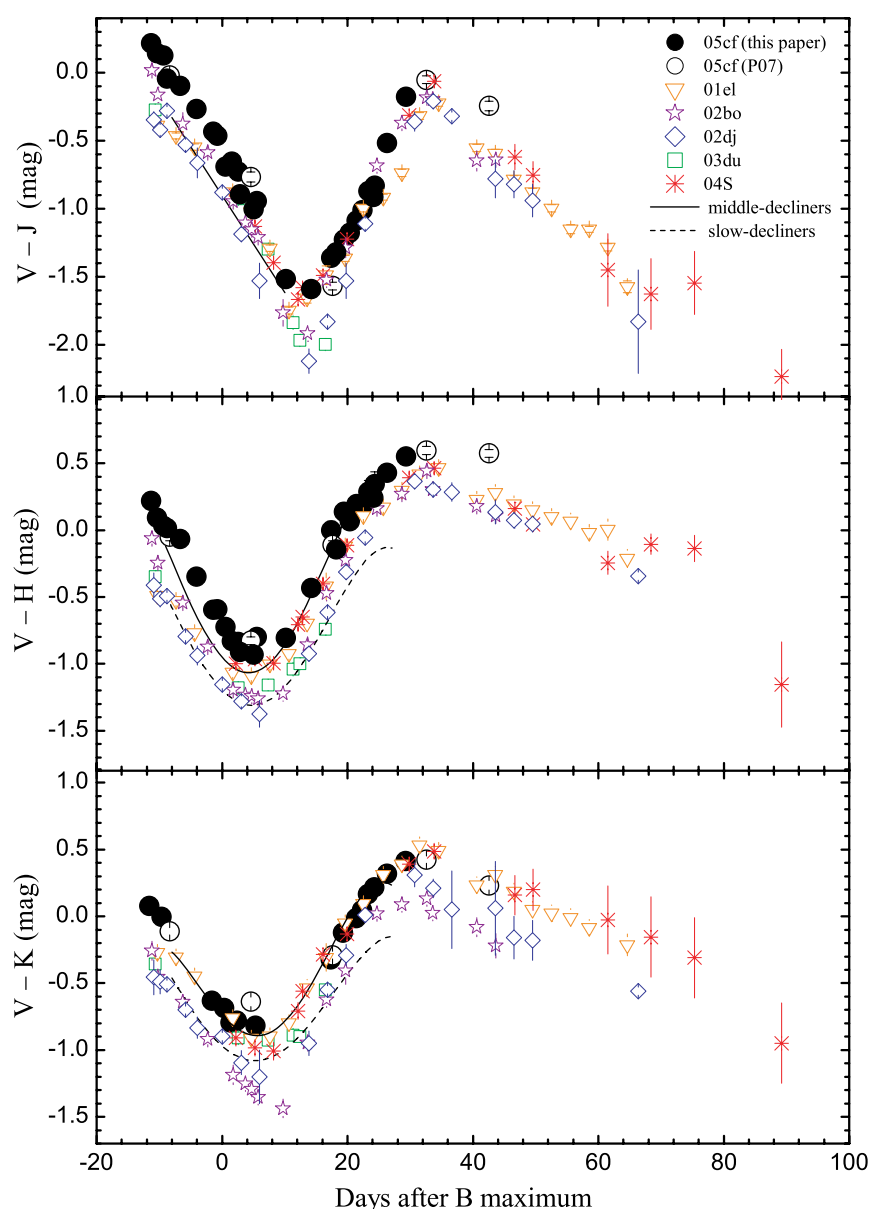

Figure 14. $V-J H K$ color curves of SN 2005cf, together with those of SNe $2001 \mathrm{el}, 2002 \mathrm{bo}, 2002 \mathrm{dj}, 2003 \mathrm{du}$, and 2004S. The dashed lines represent the mean loci of the unreddened SNe Ia with $\Delta m_{15}=0.8-1.0 \mathrm{mag}$; the solid lines denote those with $\Delta m_{15}=1.0-1.3 \mathrm{mag}$ (Krisciunas et al. 2004a).

(A color version of this figure is available in the online journal.)

elements at ejection velocities above $\sim 16,000 \mathrm{~km} \mathrm{~s}^{-1}$, such as Fe II $\lambda \lambda 2346,2357,2365$, and 2395, according to the syntheticspectrum fit by Kirshner et al. (1993). The complex of iron-peak element line blending might also explain the less prominent, absorption-like feature in the SED blueward of $2500 \AA$ at days 42 and 45. As SN 1992A suffers negligible reddening from interstellar material in both the Milky Way and the host galaxy (e.g., Wang et al. 2006; Jha et al. 2007), the observed absorption feature around $2300 \AA$ is not caused by novel dust extinction. We suggest that the flux deficit in the F220W/uvm2 filter is likely an indication of the spectral evolution common to normal SNe Ia.

\subsection{The Color Curves}

Figure 13 shows the optical color curves of SN 2005cf ( $U-B$, $B-V, V-R$, and $V-I)$, corrected for the reddening derived in Section 3.6. Also overplotted are the color curves of the Type Ia SNe 2001el, 2002bo, 2002dj, 2003du, and 2004S, all corrected for reddening in both the Milky Way and the host galaxies.

After a rapid decline at early phases, the $U-B$ color of SN $2005 \mathrm{cf}$ reached a minimum at $t \approx-5$ days and then became progressively redder in a linear fashion until $t \approx+23$ days, when the color curves entered a plateau phase (Figure 13(a)). SN 2003du exhibited similar behavior. The overall color of SN $2005 \mathrm{cf}$ is redder than that of SNe 2001el and 2003du but bluer than that of SN 2004S. The scatter in $U-B$ at maximum can reach \pm 0.2 mag between SNe Ia having similar values of $\Delta m_{15}$, suggesting that a large uncertainty might be introduced when using this color index to estimate the reddening of SNe Ia.

The $B-V$ colors of the selected SNe (Figure 13(b)) show similar evolution, except for SN 2001el near maximum and SN 2002bo at early nebular phases. We note that SN 2001el reached the bluest color slightly later, and it is also bluer than other comparison $\mathrm{SNe} I a$ at this point, as with $U-B$. The $B-V$ color of SN 2002bo after $t=+40$ days evolves at a faster pace than the Lira-Phillips relation (Phillips et al. 1999) because of the flatter photometric evolution in the $B$ band. This serves as a reminder that the Lira-Phillips relation does not hold for all SNe Ia, specifically the fast-expanding events (Wang et al. 2008a) and the SN 2000cx-like objects (Li et al. 2001).

The $V-R$ and $V-I$ color curves of SN 2005cf (Figures 13(c) and (d)) exhibit a behavior that is quite similar to that of the normal SNe Ia. By contrast with other normal SNe, SNe 2002bo and 2002dj are very blue in $V-I$ (and possibly in $V-R$ ), and they also reach their blue peak in $V-I$ about 4 days earlier.

Figure 14 shows the observed $V-J H K$ colors of SN 2005cf, together with the comparison $\mathrm{SNe}$ Ia corrected for reddening. The $V-J$ and $V-H$ colors are redder than the average values of the comparison $\mathrm{SNe}$ Ia by $\sim 0.2 \mathrm{mag}$, but the $V-K$ color shows little difference. As with $V-I$, SNe 2002bo and 2002dj are bluer in all of the $V-J H K$ colors. We notice, however, that most of the color difference would disappear, were a smaller total-to-selective absorption ratio applied to their extinction corrections $\left(R_{V}=2.0\right.$ rather than 3.1). This suggests that either the NIR luminosities of the fast-expanding events were relatively faint with respect to the optical luminosities, or a lower $R_{V}$ value is required for their dust extinction (Wang et al. 2009, in preparation).

The overall color evolution of SN 2005cf closely resembles the selected normal SNe Ia with similar $\Delta m_{15}$. Note that $\mathrm{SNe}$ 2002 bo and 2002dj exhibit distinctly bluer $V-I J H K$ colors with $R_{V}=3.1$.

\subsection{Interstellar Extinction}

The Galactic extinction toward SN 2005cf is $A_{V}^{\mathrm{Gal}}=0.32 \mathrm{mag}$ (Schlegel et al. 1998), corresponding to a color excess of $E(B-V)=0.097$ mag adopting the standard reddening law of Cardelli et al. (1989). In this section, we use several empirical methods to derive the host-galaxy reddening of SN 2005cf. All methods assume that SN 2005cf has intrinsic colors similar to those of normal SNe Ia, with either similar evolution in some colors or comparable $\Delta m_{15}$ values.

Phillips et al. (1999) proposed correlations between the lightcurve width parameter $\Delta m_{15}$ and the intrinsic $B_{\max }-V_{\max }$ or $V_{\max }-I_{\max }$ values (or $C_{\max }$ ). The $B-V$ color at early nebular phases (30 days $\lesssim t \lesssim 90$ days] was found to evolve in a similar fashion for most SNe Ia (the Lira-Phillips relation; Lira 1995; Phillips et al. 1999), allowing one to statistically separate the reddening from the intrinsic color component. In addition, Wang et al. (2005) suggested using the $B-V$ color at 12 days after $B$ maximum $\left[(B-V)_{12}\right.$, or $\left.C_{12}\right]$ as a reddening indicator because the intrinsic value of this postmaximum color was found to be a tight function of $\Delta m_{15}$. Based on the LiraPhillips relation, Jha et al. (2007) also proposed to use the color at $t=35$ days to measure the host-galaxy reddening of $\mathrm{SNe}$ Ia.

As shown in Figure 15, the empirical relations between the observed colors and $\Delta m_{15}$ were recalibrated using 28 


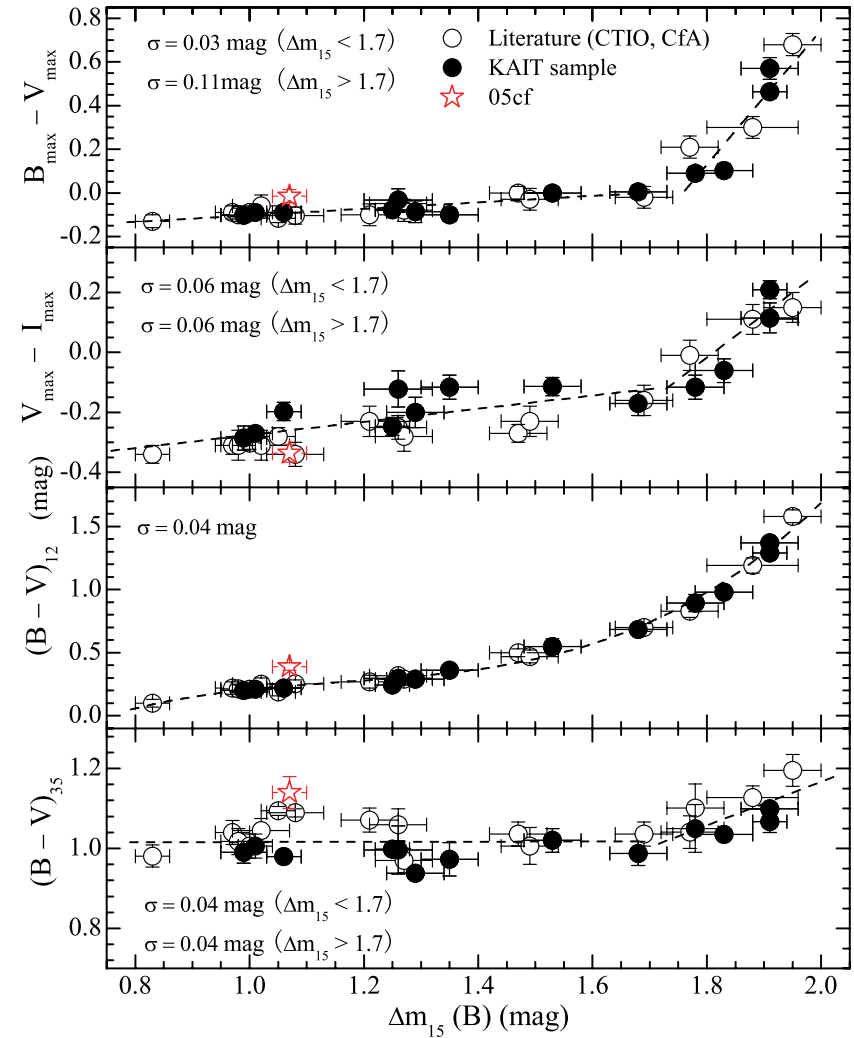

Figure 15. Correlation between the decline rate $\Delta m_{15}$ and the observed colors for low-reddening SNe Ia. Open circles represent the sample collected from the literature (Hamuy et al. 1996a, 1996b; Riess et al. 1999; Riess et al. 2005; Jha et al. 2006b; Garnavich et al. 2004; Patat et al. 1996; Salvo et al. 2001; Krisciunas et al. 2004a); filled circles show those from the KAIT photometry (M. Ganeshalingam et al. 2009, in preparation). The observed colors of SN 2005cf, corrected for the Galactic reddening, were also overplotted (star symbol).

(A color version of this figure is available in the online journal.)

well-observed, low-reddening $\mathrm{SNe} \mathrm{Ia},{ }^{22}$ of which 15 are from the literature and 13 are from the new KAIT photometric database (M. Ganeshalingam et al. 2009, in preparation). The selected SN sample closely follows the Lira-Phillips relation (e.g., with a mean slope of $-0.011 \pm 0.001 \mathrm{mag} \mathrm{day}^{-1}$ and with the measured $\left.E(B-V)_{\text {host }} \lesssim 0.05 \mathrm{mag}\right)$. With the above sample, the coefficients for the $C_{\max }-\Delta m_{15}$ relation were determined and are reported in Table 11 .

Our determinations are generally comparable to the earlier results by Phillips et al. (1999), but with a slightly steeper slope in $B_{\max }-V_{\max }$ and bluer unreddened zero points. Note that the $\left(B_{\max }-V_{\max }\right)-\Delta m_{15}$ correlation breaks down at large $\Delta m_{15}$ values; a much steeper slope is required for very fast decliners. The $V_{\max }-I_{\max }$ color does not show a great correlation with the decline rate, with a root-mean square (rms) scatter of $\sim 0.06$ mag. Applying the $C_{\max }-\Delta m_{15}$ relation to $\mathrm{SN} 2005 \mathrm{cf}$, we obtain $E(B-V)_{\max }=0.06 \pm 0.04$ mag and $E(V-I)_{\max }=$ $-0.06 \pm 0.06 \mathrm{mag}$. From the $B-V$ color of SN $2005 \mathrm{cf}$ in the nebular phase, we measured $E(B-V)_{\text {tail }}=0.13 \pm 0.04 \mathrm{mag}$.

The $C_{12}-\Delta m_{15}$ relation was also reexamined with the new sample. The zero point of the intrinsic color at the nominal decline rate is bluer than in previous reports (Wang et al. 2006) by $0.06 \mathrm{mag}$. This difference is perhaps due to the stricter

22 SNe 1992A, 1992al, 1992bc, 1992bl, 1992bo, 1993H, 1993O, 1994D, 1994S, 1996X, 1998aq, 1998bp, 1998de, 1999by, 1999ej, 2000ca, 2000dk, $2000 \mathrm{dr}, 2001 \mathrm{ba}, 2002 \mathrm{dl}, 2002 \mathrm{ha}, 2002 \mathrm{fk}, 2003 \mathrm{gs}, 2003 \mathrm{hv}, 2003 \mathrm{du}, 2004 \mathrm{at}$, $2005 \mathrm{el}$, and $2005 \mathrm{ki}$.

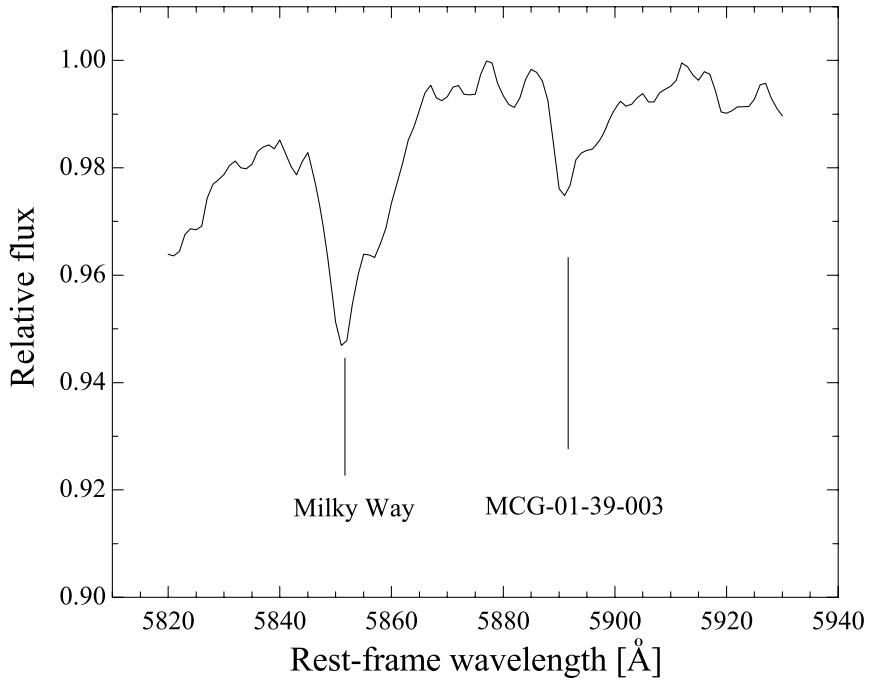

Figure 16. Interstellar Na I D region in the composite spectrum of SN 2005cf obtained from all spectra within 5 days of maximum brightness. The components due to the Milky Way and the host galaxy are clearly seen.

criteria of selecting the training sample and also indicates the difficulty of separating reddening from the intrinsic color. This postmaximum color index gives $E(B-V)_{12}=0.12 \pm 0.04$ mag.

In comparison with the sample observed with other systems, the KAIT sample of 13 SNe Ia seems to be slightly bluer by $\sim 0.05$ mag at $t=35$ days (see the bottom panel in Figure 15), while this difference does not hold for the other color indices shown in the same plot. Such a discrepancy may arise from systematic effects, such as the $S$-corrections, which were found to show noticeable divergence in the nebular phase (see Figure $3)$. In addition, we point out that the $(B-V)_{35}$ color may not be a constant for the fast decliners; a larger $\Delta m_{15}$ corresponds to a redder $(B-V)_{35}$. With the new unreddened loci, we measured $E(B-V)_{35}=0.13 \pm 0.05 \mathrm{mag}$ for SN $2005 \mathrm{cf}$.

Krisciunas et al. (2000, 2004a) have shown that the intrinsic $V-J H K$ colors of SNe Ia are uniform and can be used as reddening indicators. Based on Krisciunas unreddened loci (see Figure 14), for SN 2005cf we obtain $E(V-J)=0.28 \pm 0.08$ mag, $E(V-H)=0.25 \pm 0.06 \mathrm{mag}$, and $E(V-K)=0.16 \pm 0.06$ mag. This corresponds to an $E(B-V)$ reddening of $0.13 \pm 0.08$ $\mathrm{mag}, 0.10 \pm 0.06 \mathrm{mag}$, and $0.06 \pm 0.06 \mathrm{mag}$, respectively.

The color excesses of SN 2005cf derived from various empirical methods are summarized in Table 12. They are consistent with each other within the uncertainties, except the $V-I$ color which shows larger scatter and may be not a reliable reddening color index. The mean value obtained by excluding the measurement from $V_{\max }-I_{\max }$ is $E(B-V)_{\text {host }}=0.10 \pm 0.03$ mag, indicating that SN 2005cf suffers small but non-negligible reddening in the host galaxy. The presence of host-galaxy reddening is also favored by studying the interstellar absorption region. As shown in Figure 16, the interstellar $\mathrm{Na}$ I D lines from the host galaxy and the Milky Way are clearly seen in the spectrum of SN 2005cf. Although the strength of the hostgalaxy component seems weaker than that from the Milky Way $(\mathrm{EW}(\mathrm{Na}$ I D $) \approx 0.22 \AA$ versus $\sim 0.42 \AA$, respectively), they may be associated with a similar amount of dust reddening; there is uncertainty in the dust-to-gas ratio, or large scatter in the $\mathrm{EW}(\mathrm{Na}$ I D) versus $E(B-V)$ relation for $\mathrm{SNe}$ Ia (e.g., Turatto et al. 2003; Blondin et al. 2009). 
Table 12

Host-Galaxy Reddening of SN 2005cf

\begin{tabular}{lcc}
\hline \hline Method & $E(B-V)_{\text {host }}(\mathrm{mag})$ & Ref \\
\hline$B_{\max }-V_{\max }$ & $0.06 \pm 0.04$ & 1,2 \\
$V_{\max }-I_{\max }$ & $-0.06 \pm 0.06$ & 1,2 \\
$(B-V)_{12}$ & $0.12 \pm 0.04$ & 3,2 \\
$(B-V)_{35}$ & $0.13 \pm 0.05$ & 4,2 \\
$(B-V)_{\text {tail }}$ & $0.13 \pm 0.04$ & 1 \\
$V-J$ & $0.13 \pm 0.08$ & 5 \\
$V-H$ & $0.10 \pm 0.06$ & 5 \\
$V-K$ & $0.06 \pm 0.06$ & 5 \\
Mean & $0.10 \pm 0.03$ & $\cdots$
\end{tabular}

Notes. Uncertainties are $1 \sigma$

(1) Phillips et al. (1999); (2) this paper; (3) Wang et al. (2005); (4) Jha et al. (2007); (5) Krisciunas et al. (2004a).

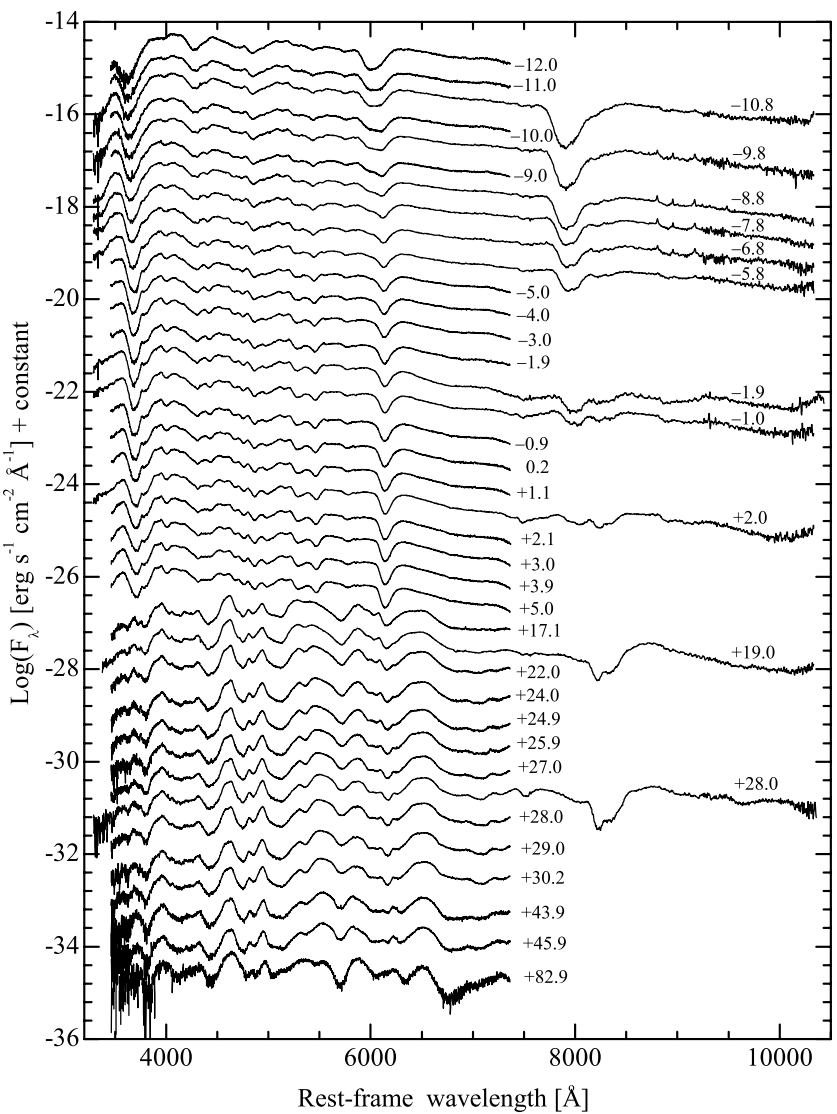

Figure 17. Optical spectral evolution of SN 2005cf. The spectra have been corrected for the redshift of the host galaxy $\left(v_{\text {hel }}=1967 \mathrm{~km} \mathrm{~s}^{-1}\right)$ but not for reddening, and they have been shifted vertically by arbitrary amounts for clarity. The numbers on the right-hand side mark the epochs of the spectra in days after $B$ maximum.

\section{OPTICAL SPECTRA}

There are a total of 38 optical spectra of SN 2005cf obtained at Lick Observatory and FLWO, spanning from $t=-12.0$ to $t=+83.0$ days with respect to the time of $B$-band maximum brightness. The complete spectral evolution is displayed in Figure 17, and two late-time nebular spectra are presented in Figure 19. The earliest spectra show very broad and highly blueshifted absorption features at $3700 \AA$ (Ca II H\&K), 6020 $\AA$ (Si II $\lambda 6355)$, and $7900 \AA$ (Ca II NIR triplet). In particular, a flat-bottomed feature is distinctly seen in $\mathrm{Si}$ II $\lambda 6355$. The spectral evolution near maximum brightness generally follows that of normal SNe Ia, with the distinctive "W"-shaped S II lines near $5400 \AA$ and the blended lines of Fe II and Si II near 4500 $\AA$. We discuss in detail the spectral evolution of SN $2005 \mathrm{cf}$ in the following subsections.

\subsection{Temporal Evolution of the Spectra}

In Figure 18, we compare the spectra of SN 2005cf with those of SNe Ia having similar $\Delta m_{15}$ at four different epochs $(t \approx-11,-7,0$, and 18 days past $B$ maximum). All spectra have been corrected for reddening and redshift. For SN 2001el, $R_{V}=2.1$ is assumed according to the analysis by Krisciunas et al. (2007). For the other SNe, the host-galaxy reddening is measured using the empirical correlations presented in Table 11 and the extinctions are corrected using the standard value $R_{V}=3.1$. The line identifications adopted here are taken from Branch et al. (2005, 2006).

At $t \approx-11$ days, the earliest spectrum of SN 2005cf is characterized by lines of singly ionized intermediate-mass elements ( $\mathrm{Si}, \mathrm{S}, \mathrm{Mg}$, and $\mathrm{Ca}$ ). The absorption lines of $\mathrm{Si}$ II $\lambda 6355$ and the Ca II NIR triplet in SN 2005cf are very broad and deep, comparable to those in SNe 2001el and 2002bo. Another interesting feature is the flat-bottomed profile of Si II $\lambda 6355$, which was previously only observed in SN $1990 \mathrm{~N}$ at $t=-14$ days and in SN 2001el at $t=-9$ days. In contrast, SNe 2003du and $2005 \mathrm{cg}$ displayed a triangular-shape feature at similar epochs. By $t \approx-6$ days, the broad absorption feature associated with Si II $\lambda 6355$ becomes narrower and evolves as a normal profile in SN 2005cf and SN 2001el. The Ca II NIR triplet weakens and shows a double minimum on the red side of the main absorption in the above two events. Such spectral evolution is also clearly seen in SN 2003du, while it is less noticeable in SN $2005 \mathrm{cg}$ at comparable phase. Around maximum brightness, the blue absorption component of the Ca II NIR triplet is still very strong in SNe 2005cf, 2001el, and 2004S, but it becomes much less prominent in SN 2003du. The O I $\lambda 7773$ line strengthens in all cases in our sample. We measured the line-strength ratio of Si II $\lambda \lambda 5958,5979$ to $\mathrm{Si}$ II $\lambda 6355$, known as $R$ (Si II) (Nugent et al. 1995), to be $0.28 \pm 0.04$ for SN $2005 \mathrm{cf}$ near maximum, in good agreement with the measurement reported by G07. At $t \approx 18$ days, the spectral evolution of all the SNe Ia in our study appears quite similar to each other. Although the Ca II NIR triplet shows the most diverse features at early epochs, they develop into a rather smooth absorption profile by 2 weeks after $B$ maximum.

Comparison of the spectra of SNe Ia having similar $\Delta m_{15}$ values reveals that they show the most diversity at the earliest epochs, with the main features having significantly different strengths and profiles (e.g., Si II $\lambda 6355$ and the Ca II NIR triplet). In general, the overall spectral evolution of SN 2005cf at early phases closely resembles that of SN 2001el.

Two late-time nebular Keck spectra, obtained with LRIS on day +319 and with DEIMOS on day +614 , are shown in Figure 19. They do not exhibit any detectable signature of a lowvelocity hydrogen emission, though the $\mathrm{S} / \mathrm{N}$ is low, especially in the case of the day +614 spectrum (see also the deep observations of SN 2005cf performed by Leonard 2007). The overall shape of the spectrum on day +319 is very similar to that of SN $2003 \mathrm{du}$ at a similar phase. The nebular spectrum of SN 2002dj at this phase is also quite normal, without showing extra flux (or evidence for presence of an echo) at shorter wavelengths as in the extreme HV event SN 2006X (Wang et al. 2008b). We note, however, that SN 2002dj is a slightly fast-expanding object with less significant reddening (Pignata et al. 2008). 


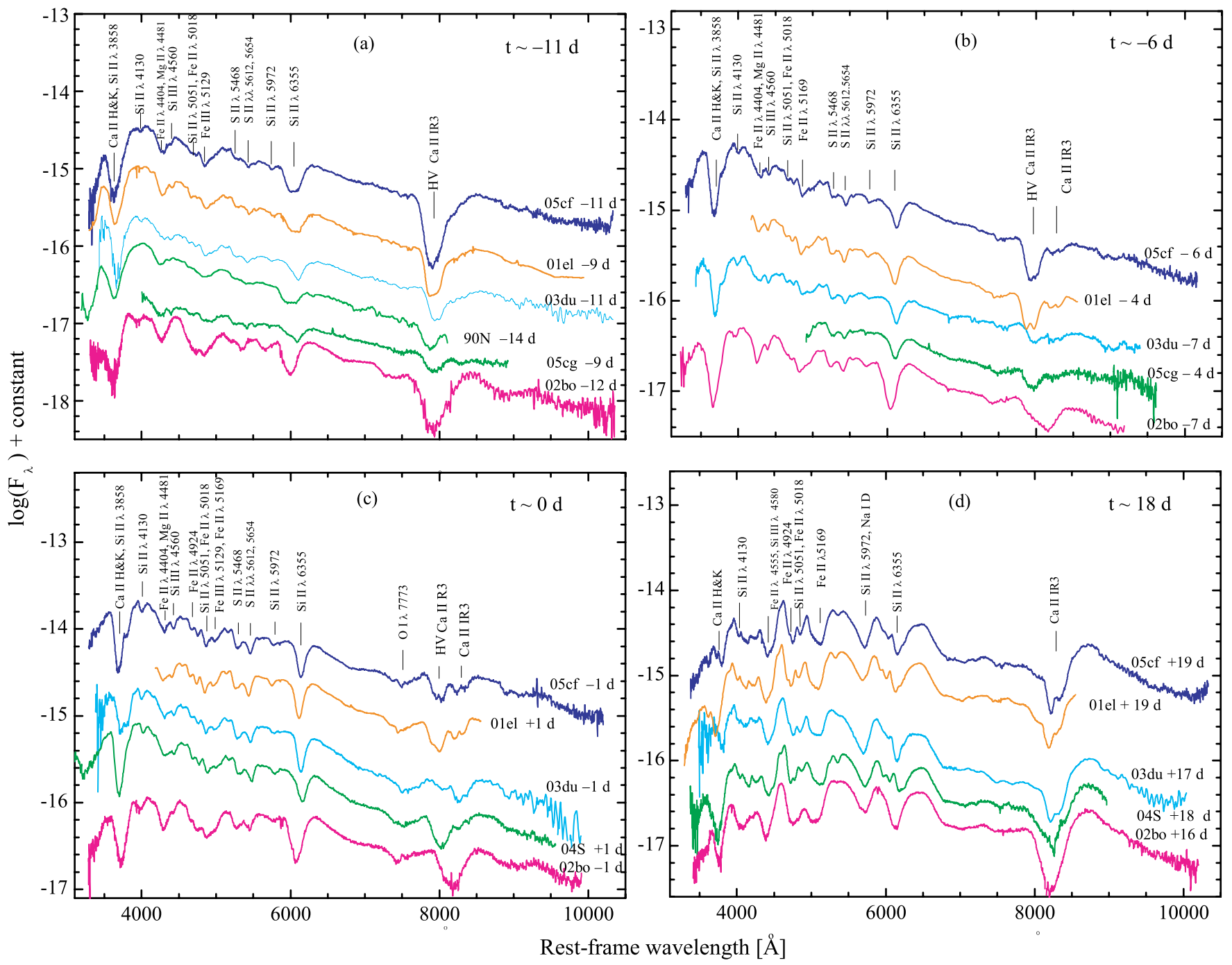

Figure 18. Spectrum of SN $2005 \mathrm{cf}$ at $-11,-6,0$, and +18 days after $B$ maximum, overplotted with the comparable-phase spectra of SNe $1990 \mathrm{~N}$ (Leibundgut et al. 1991), 2001el (Wang et al. 2003b; Mattila et al. 2005), 2002bo (Benetti et al. 2004), 2003du (Stanishev et al. 2007), 2004S (Krisciunas et al. 2007), and 2005cg (Quimby et al. 2006). All spectra shown here have been corrected for the reddening and redshift of the host galaxy. For clarity of display, the spectra were arbitrarily shifted in the vertical direction.

(A color version of this figure is available in the online journal.)

\subsection{The Photospheric Expansion Velocity}

In this subsection, we examine the blueshift of the absorption minimum of some spectral features in SN 2005cf. The location of the blueshifted minima may approximately represent that of the photosphere at earlier SN phase. In measuring the absorption minimum, both the Gaussian fit routine within IRAF as well as the direct measurement (by eye) of the center of the absorption were applied, and the results were averaged. The derived velocities ${ }^{23}$ of SN $2005 \mathrm{cf}$ from the Si II $\lambda 6355$ and S II $\lambda 5640$ lines as a function of time are shown in Figure 20, together with those of the comparison SNe Ia. The measurements from the spectra published by G07 are also overplotted (small filled circles). All velocities have been corrected for the redshifts of the host galaxies.

At the earliest phases, the expansion velocity implied from Si II $\lambda 6355$ for SN $2005 \mathrm{cf}$ is $\sim 2000 \mathrm{~km} \mathrm{~s}^{-1}$ higher than that for SNe 1990N (Leibundgut et al. 1991), 1998aq (Branch et al. 2003), and 2003du (Stanishev et al. 2007), and comparable to

23 The relativistic effect was always taken into account when calculating the ejecta velocities of the SN. that for SNe 1994D (Filippenko 1997; Patat et al. 1996) and 2001el (Wang et al. 2003a, 2003b; Mattila et al. 2005). This velocity declines very rapidly within the first two weeks before $B$ maximum and then maintains a plateau phase for about a month. Such evolution may be related to the fact that the Si II absorption region is close to the photosphere at earlier phases but becomes more detached at later times (Patat et al. 1996). In contrast, the velocity derived from the $\mathrm{S}$ II $\lambda 5640$ line is slightly lower than that of the other $\mathrm{SNe}$ Ia and shows a flat evolution from $t \approx-12$ days to -5 days. SN $2001 \mathrm{el}$ may show a similar plateau feature, though the data are sparse.

Following Benetti et al. (2005), we calculate the velocity gradient $\dot{v}$ of Si II $\lambda 6355$ for SN $2005 \mathrm{cf}$ as $38 \pm 5 \mathrm{~km} \mathrm{~s}^{-1}$ day $^{-1}$ during the period $t \approx 0-30$ days, which puts SN 2005cf in the group of normal SNe Ia having low-velocity gradients (LVGs).

\subsection{The High-Velocity Features}

In addition to the evolution of the photospheric expansion, the well sampled spectra of SN 2005cf (especially those at earlier phases) provide a good opportunity to study the HV features that were only seen in the earliest spectra. The HV material is 


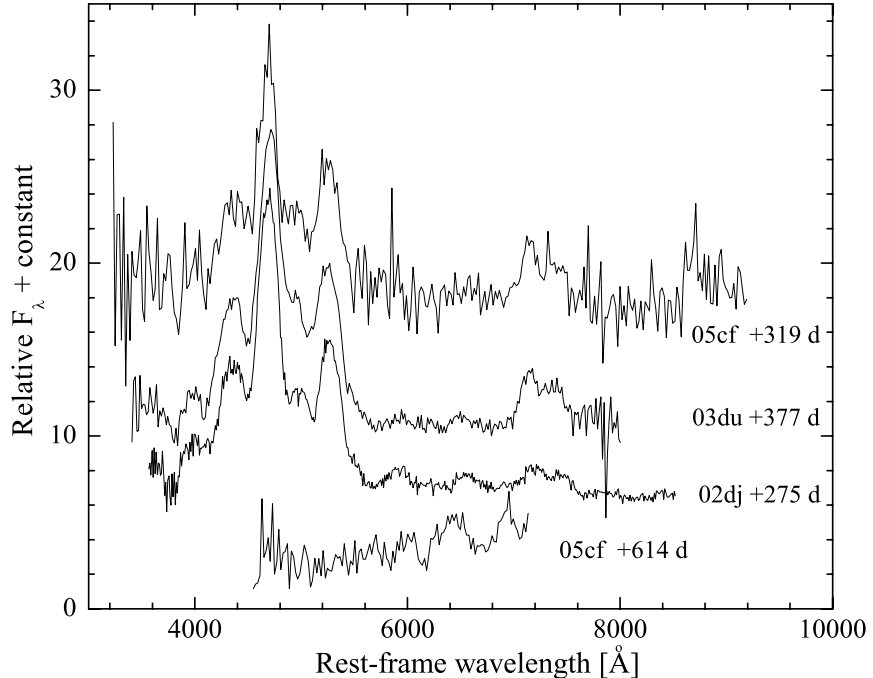

Figure 19. Late-time nebular spectra of SN 2005cf, slightly smoothed. The nebular spectra of SN 2003du (Stanishev et al. 2007) and SN 2002dj (Pignata et al. 2008) at about the same phase as the day 319 SN 2005cf spectrum are shown for comparison. Both reddening and redshift corrections have been applied to the spectra.

usually located in the outermost layers of the ejecta where SNe Ia show the highest degree of heterogeneity.

\subsubsection{The High-Velocity Si II}

Figure 21 (left panel) shows the premaximum evolution of the velocity-space distribution of Si II $\lambda 6355$ in the spectra of SN 2005cf. In the -12 day spectrum, this absorption profile is broad and asymmetric with a stronger minimum on the blue side. At $t=-11$ days, the Si II line exhibits a flat-bottomed feature with comparable absorption minima on both red and blue sides. As the spectrum evolves, the red-side minimum gradually dominates. By $t=-6$ days, the Si II absorption feature gradually develops into a single minimum with a typical velocity of $\sim 10,500 \mathrm{~km} \mathrm{~s}^{-1}$, though the line profile may still be affected by the blue component until around maximum light (G07). The overall evolution of the line profile clearly shows the presence of another HV absorption component.

The observed line profiles of Si II $\lambda 6355$ at earlier phases can be well fit by a double-Gaussian function with separate central wavelengths. The HV component could be a thin, pure Si shell or a mixed layer of Si II and C II $\lambda 6580$ (Fisher et al. 1997; Mazzali et al. 2001, 2005). Assuming that the blue-side absorption component is primarily produced by the HV Si II detached from the photosphere, the mean absorption-minimum velocity is estimated to be $18,000 \pm 500 \mathrm{~km} \mathrm{~s}^{-1}$ during the period from $t=-12$ days to $t=-7$ days (see also the open circles in Figure 20). This velocity is much higher than the value inferred from the absorption minimum on the red side.

The presence of HV Si II in SN 2005cf was also proposed by G07 by modeling the observed spectra with the use of the parameterized code for SN synthetic spectroscopy, SYNOW (Fisher 2000). Their analysis suggested that the HV feature of Si II $\lambda 6355$ in SN $2005 \mathrm{cf}$ is detached at $19,500 \mathrm{~km} \mathrm{~s}^{-1}$. Given the contamination by such an HV Si II feature, the photospheric velocity measured directly from the overall line profile was overestimated. The recomputed $v_{\exp }$ values from the Si II line (see the gray dots in Figure 20), after removing the HV component, happen to match the velocity evolution predicted from the 1/3 solar metallicity model (e.g., Lentz et al 2000).

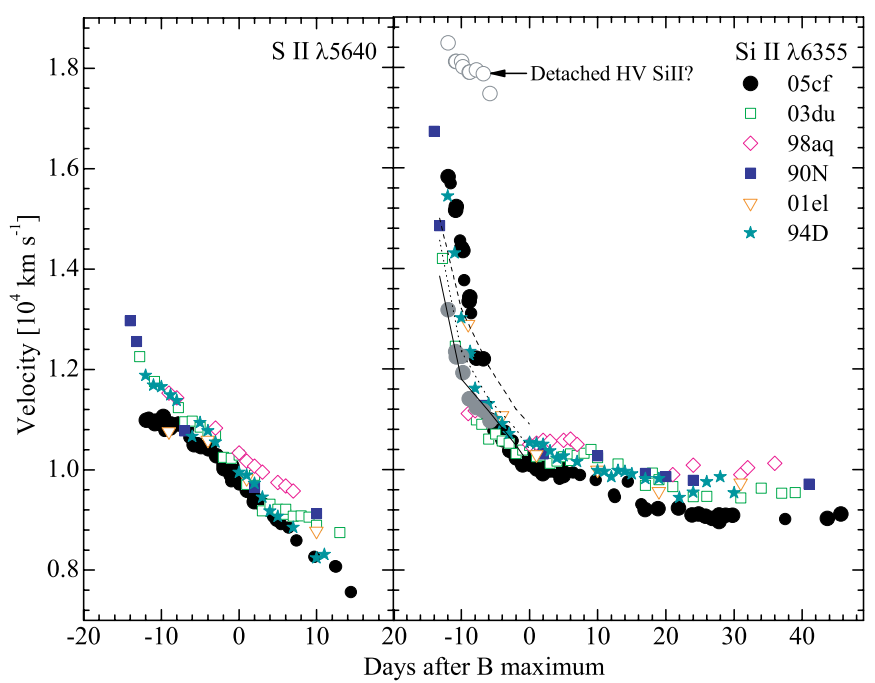

Figure 20. Evolution of the expansion velocity of SN 2005cf as measured from the minimum of $\mathrm{Si}$ II $\lambda 6355$ and $\mathrm{S}$ II $\lambda 5640$, compared with corresponding values for SNe 1990N, 1994D, 1998aq, 2001el, and 2003du (see text for references). The gray dots show the low-velocity component decomposed from the Si II $\lambda 6355$ absorption of SN 2005 cf by using the double-Gaussian model (see Section 4.3.1 and Figure 21); the open circles represent the detached HV component. Overplotted are velocities predicted by the Lentz et al. (2000) model for cases of $\times 10$ (dashed line), $\times 3$ (dotted line), and $\times 1 / 3$ (solid line) solar metallicity in the $\mathrm{C}+\mathrm{O}$ layer.

(A color version of this figure is available in the online journal.)

In the right-hand panels of Figure 21, we also compare the Si II absorption features in the earliest spectra. The presence of the HV feature of $\mathrm{Si}$ II has also been suggested in SNe 1990N, 1999ee, 2001el, and 2005cg (Mazzali et al. 2001; Mattila et al. 2005; Quimby et al. 2006), but none of them could be securely established due to the sparse coverage of the premaximum spectra. Of the above SNe Ia, the flat-bottomed profile of the $\mathrm{Si}$ II line is seen only in SN $1990 \mathrm{~N}$ at $t=-14$ days and SN 2001el at $t=-9$ days. Inspection of the $t=-13$ day spectrum of SN 2003du reveals an Si II absorption reminiscent of the feature seen in SN 2005cf at $t=-10$ days. A triangularshaped profile is present in the spectra of all the other events. It is therefore likely that such a "peculiar" line profile is just a snapshot of the common evolutionary pattern (see similar arguments by Stanishev et al. 2007).

\subsubsection{The High-Velocity Ca II}

Figure 22 presents the detailed evolution of the Ca II NIR triplet and Ca II H\&K of SN 2005cf. In comparison with the $\mathrm{Si}$ II line, the HV features in the Ca II NIR triplet are more frequently observed in SNe Ia (e.g., Mazzali et al. 2005), as they are more pronounced and may have a longer duration. The HV component dominates in the Ca II NIR lines at earlier phases, but it gradually loses its strengths with time. In the Ca II NIR triplet, the HV components are more separated from the photospheric components than in the Si II line. At $t=-11$ days, the HV component shows an expansion velocity at about $23,000 \mathrm{~km} \mathrm{~s}^{-1}$. By $t=+2$ days, the velocity is around $19,000 \mathrm{~km}$ $\mathrm{s}^{-1}$ and the absorption minimum is beginning to be dominated by the photospheric component at $\sim 10,000 \mathrm{~km} \mathrm{~s}^{-1}$.

The Ca II H\&K line may show similar HV features, but they overlap with the Si II $\lambda 3858$ line at earlier phases. Due to the severe line blending, it is difficult to disentangle the HV component and quantify its strength. Assuming a doubleGaussian model, we measure the velocity of the HV component to be $\sim 25,500 \mathrm{~km} \mathrm{~s}^{-1}$ and $20,000 \mathrm{~km} \mathrm{~s}^{-1}$ in the $t=-11$ day 

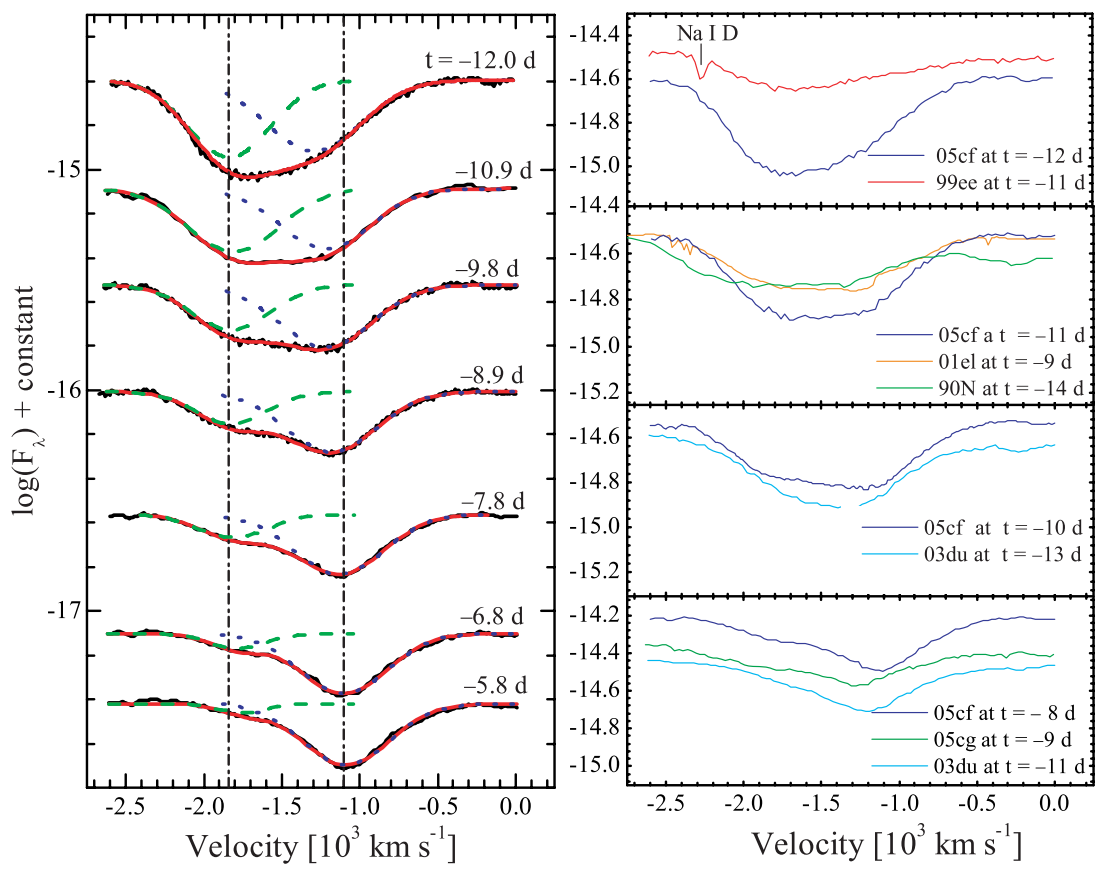

Figure 21. Left panel: evolution in velocity space of Si II $\lambda 6355$ in SN $2005 \mathrm{cf}$ compared with the double-Gaussian fit. Dashed green lines show the velocity distribution on the blue side, and dotted blue lines show the component on the red side. The red solid lines represent the best-fit curve to the observed profile. Right panels: comparison of premaximum evolution of the Si II $\lambda 6355$ profile of SN 2005cf with that of SNe 1990N, 1999ee, 2001el, 2003du, and 2005cg at four selected epochs (see text for references).

(A color version of this figure is available in the online journal.)

and -1 day spectra, respectively, similar to those measured for the Ca II NIR triplet. The higher velocity of the HV component in the $\mathrm{Ca}$ II lines with respect to the $\mathrm{Si}$ II line perhaps suggests a different abundance distribution in the ejecta.

\subsubsection{Origin of the HV Features}

Currently, the origin of the HV features is debated. In principle, their formation can be due to an abundance and/ or a density enhancement in the outer-layer ejecta. According to Mazzali et al. (2005), an abundance enhancement alone may not account for the observed HV features, as the nuclear burning cannot produce enough $\mathrm{Si}$ and $\mathrm{Ca}$ required in the outer region. In contrast, an enhancement in the local density can well reproduce the HV spectral evolution, as demonstrated by the three-dimensional explosion models (e.g., Röpke et al. 2006). One possible scenario for the density enhancement is the interaction of the outermost ejecta with circumstellar matter (CSM) produced in the vicinity of the SN (e.g., Gerardy et al. 2004). On the other hand, an aspherical ejecta model could also cause the HV density enhancement. Variations of the strength of the HV features may be explained by different viewing angles if they result from aspherical structures like a torus or clumps (Tanaka et al. 2006).

Stanishev et al. (2007) proposed that the strength of the HV components in the Si II and $\mathrm{Ca}$ II lines may be correlated. Inspection of the early-time spectra presented in Figure 18 suggests that such a correlation holds true for SN $2005 \mathrm{cf}$ and all the comparison objects, though their strength varies from SN to SN. This perhaps favors the origin of the observed HV features from either CSM interaction or aspherical structure of the ejecta produced by the explosion itself. Note that the detached HV features discussed here seem different from those observed in SN 2006X or even in SN 2002bo; however, the same configuration with a much larger photospheric velocity may explain the difference (Tanaka et al. 2008).

\section{THE DISTANCE AND LUMINOSITY OF SN 2005CF}

The extensive photometric observations of SN 2005cf, from the UV to the NIR bands, enable us to construct the uvoir "bolometric" light curve within 0.2-2.4 $\mu \mathrm{m}$. For this calculation, we used the normalized passband transmission curves given by Bessell (1990). The integrated flux in each filter was approximated by the mean flux multiplied by the effective width of the passband, and was corrected for the reddening. Since the filter transmission curves do not continuously cover the spectrum and some also overlap, we corrected for these gaps and overlaps by adjusting the effective wavelengths of the filters in the UV, optical, and NIR passbands.

\subsection{The Distance to SN 2005 cf}

The distance to SN 2005cf is important for deriving the bolometric luminosity. Direct measurements were unavailable in the literature, so we used several methods to estimate the distance. According to Wang et al. (2006), a nominal standard SN Ia with Cepheid-based calibration has an absolute peak magnitude of $-19.27 \pm 0.05 \mathrm{mag}$ in the $V$ band. Combining this value with the fully corrected apparent peak magnitude of SN 2005cf, we obtain a distance modulus $\mu=32.29 \pm 0.12$ mag. We also determine the distance using the latest version of the MLCS2k2 fitting technique (Jha et al. 2007), which yields $\mu=32.43 \pm 0.13 \mathrm{mag}$.

Krisciunas et al. (2004b) propose that $\mathrm{SNe}$ Ia are more uniform in the NIR bands than in the optical; their NIR peak luminosities are found to be nearly independent of the lightcurve shape. The absolute NIR peak magnitudes for SNe Ia with $\Delta m_{15}<1.7$ are reported as -18.61 in $J,-18.28$ in $H$, and -18.44 mag in $K$, respectively. Assuming the same NIR magnitudes for SN 2005cf, we derive a mean distance modulus $\mu=32.20 \pm 0.10 \mathrm{mag}$. 




Figure 22. Premaximum evolution in velocity space for the $\mathrm{Ca}$ II $\mathrm{H} \& \mathrm{~K}$ and NIR triplet of SN 2005cf. Vertical dashed lines indicate, respectively, the highest velocity in the earliest phase $\left(\sim 23,000 \mathrm{~km} \mathrm{~s}^{-1}\right)$ and the photospheric velocity (at $\sim 11,000 \mathrm{~km} \mathrm{~s}^{-1}$ )

(A color version of this figure is available in the online journal.)

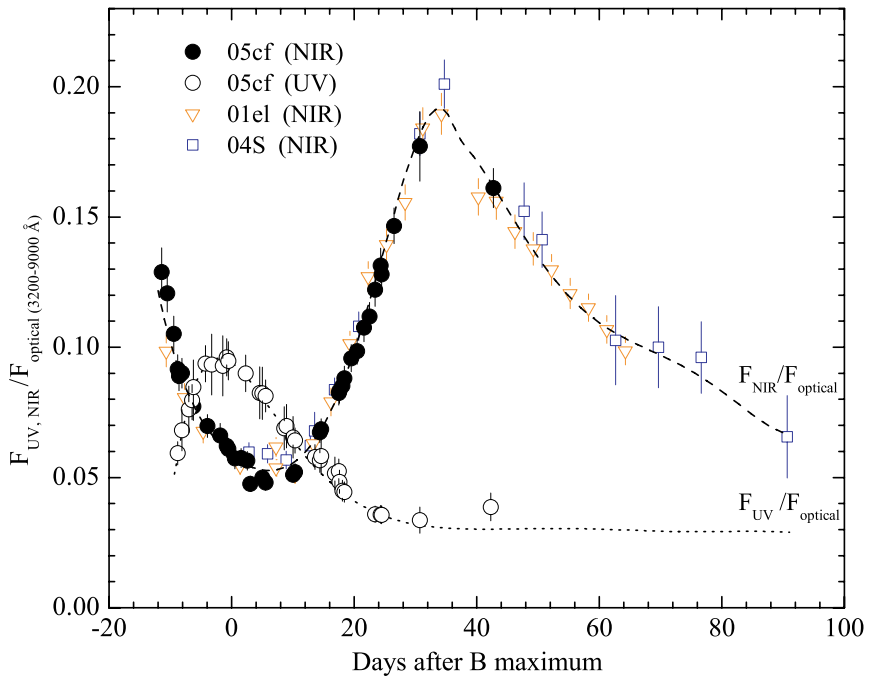

Figure 23. Ratio of the UV and NIR fluxes to the optical flux for SN 2005cf. Overplotted are the NIR flux ratios of SNe 2001el and 2004S

(A color version of this figure is available in the online journal.)

Averaging the above three distances, we obtain a weighted mean of $\mu=32.31 \pm 0.11 \mathrm{mag}$ for SN 2005cf. Note that this estimate may still suffer from an additional uncertainty of $0.12-$ $0.15 \mathrm{mag}$, due to the intrinsic luminosity dispersion of SNe Ia in both optical and NIR bands (Krisciunas et al. 2004b; Wang et al. 2006; Wood-Vasey et al. 2008).

\subsection{The Missing Flux Below the Optical Window}

The filled circles in Figure 23 show the temporal evolution of the ratio of the NIR-band emission (9000-24000 $\AA$ ) to the optical (3200-9000 $\AA$ ), $F_{\text {IR }} / F_{\text {opt }}$. The flux ratios obtained from $\mathrm{SNe} 2001 \mathrm{el}$ and $2004 \mathrm{~S}$ are overplotted. The dashed curve in the plot represents the best fit to the data of SN 2005cf. In the fit, the NIR contribution at $t>40$ days is assumed to be the same as that of SN 2001el and SN 2004S. This assumption is reasonable because the $F_{\mathrm{IR}} / F_{\text {opt }}$ ratio of SN 2005cf agrees well with the corresponding values of these two SNe Ia from the very beginning to $t \approx+40$ days. Initially, the ratio shows a sharp

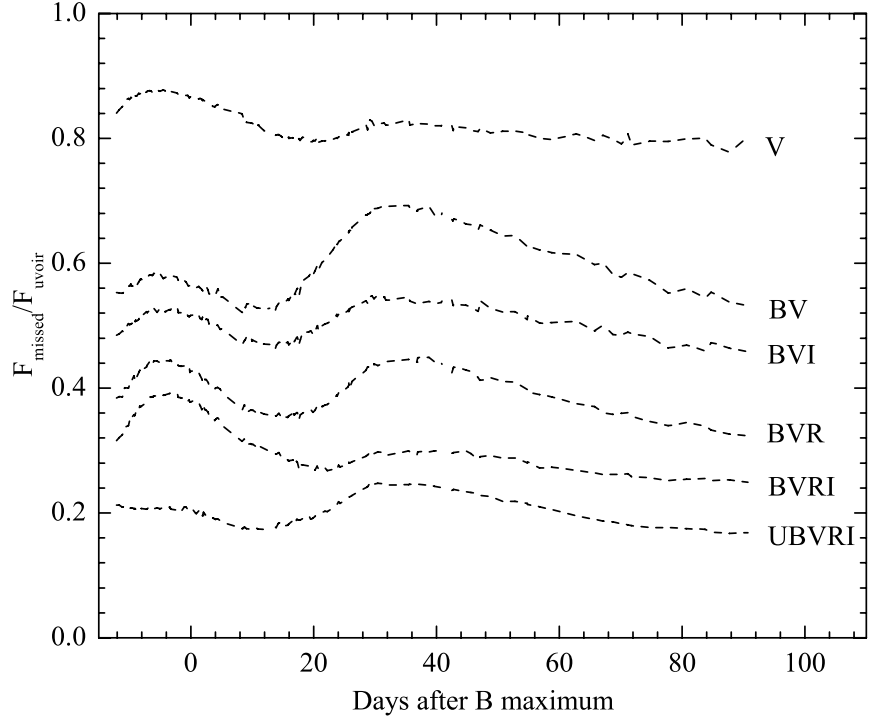

Figure 24. Correction factors for missing passbands. The correction factors are obtained by comparing the fluxes in the passbands with the total uvoir flux.

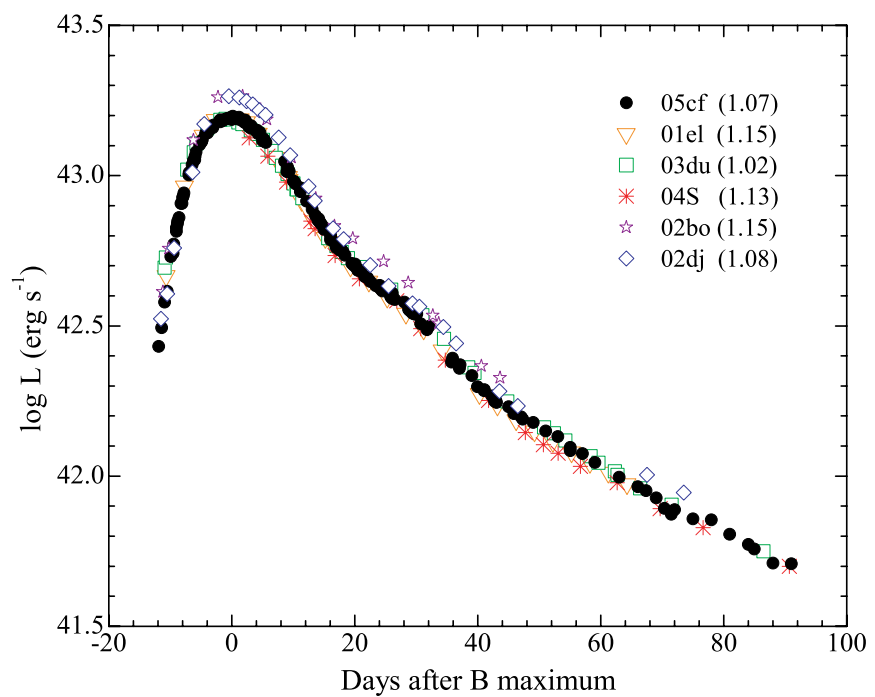

Figure 25. uvoir bolometric light curve of SN 2005cf. Overplotted are the corresponding light curves of the comparison SNe Ia. The numbers in parentheses represent the $\Delta m_{15}$ values for the SNe Ia.

(A color version of this figure is available in the online journal.)

decline with a minimum $\sim 4$ days after the $B$ maximum. Then it rises rapidly in a linear fashion and reaches a peak $(\sim 20 \%)$ at $t \approx+30$ days, when the secondary maximum appears in the NIR. At nebular phases, the NIR contribution gradually declines and becomes $<10 \%$ at $t \approx+80$ days, similar to that found by Suntzeff in studying SN 1992A (1996).

The contribution of the UV flux in the 1600-3200 $\AA$ range to the optical is shown with the open circles in Figure 23. The dotted line indicates the best fit to the observed data points, assuming that the UV flux remains constant at $t>+30$ days. A flat contribution at late times was evidenced by the UV data of SN 2001el from the HST archive (see Figure 14 in Stanishev et al. 2007). The UV contribution is found to be generally a few percent of the optical in SN 2005cf, with a peak $(\sim 10 \%)$ at around the $B$ maximum. Three weeks after maximum, the ratio remains at a level of $3 \%-4 \%$.

It is also interesting to estimate the ratio of the optical fluxes to the bolometric flux of SN 2005cf as a function of time. As most 
SNe Ia have not been observed in the UV or the NIR bands, these ratios are useful for correcting the observed optical luminosity to the bolometric luminosity. Figure 24 shows the correction factors (defined as the missing fraction of the observed flux relative to the generic uvoir bolometric luminosity) obtained from SN 2005cf; one can see that the bolometric luminosity is dominated by the optical fluxes. The missing flux beyond the optical window is about $20 \%$, with a slightly larger fraction at $t \approx+30$ days due to the appearance of the secondary maximum in the NIR. The corrections, based on the fluxes in the $U B V R I$, $B V R I, B V R, B V I, B V$, and $V$ bands, are also shown in the plot. We note that the bolometric correction for the $V$ filter exhibits the least variation with time: the overall variation is less than $4 \%$ before $t \approx+30$ days, and at later phases the correction stays nearly constant. This validates previous assumptions that the $V$-band photometry can well represent the bolometric light curve, in particular at later phases, at a constant fraction of about $20 \%$.

\subsection{The Bolometric Light Curve}

Figure 25 shows the "uvoir" bolometric light curves of SN 2005cf and several other SNe Ia. The UV emission of all the comparison $\mathrm{SNe}$ was corrected on the basis of $\mathrm{SN}$ $2005 \mathrm{cf}$. Similar corrections were applied to their light curves when the NIR observations were missing. The distances to the comparison SNe Ia were derived using the methods adopted in Section 5.1. The uvoir bolometric light curves of our SN Ia are overall very similar in shape, with the exceptions of SN 2002 bo and SN 2002dj, which seem to rise at a faster pace. The maximum bolometric luminosity of SN $2005 \mathrm{cf}$ is estimated to be $(1.56 \pm 0.20) \times 10^{43} \mathrm{erg} \mathrm{s}^{-1}$ around the $B$-band maximum, which is similar to that of SNe 2001el and 2003du but less than that of SNe 2002bo and 2002dj. However, differences in the bolometric luminosity at maximum may in part be due to errors in the absorption corrections and/or the distance modulus.

With the derived bolometric luminosity, we can estimate the synthesized ${ }^{56} \mathrm{Ni}$ mass, one of the primary physical parameters determining the peak luminosity, the light-curve width, and the spectroscopic evolution of SNe Ia (e.g., Kasen \& Woosley 2007). Assuming the Arnett law (Arnett 1982; Arnett et al. 1985; Branch \& Tammann 1992), the maximum luminosity produced by the radioactive ${ }^{56} \mathrm{Ni}$ can be written as (Stritzinger \& Leibundgut 2005)

$$
L_{\max }=\left(6.45 e^{\frac{-t_{r}}{(8.8 \mathrm{~d})}}+1.45 e^{\frac{-t_{r}}{(111.3 \mathrm{~d})}}\right)\left(\frac{M_{\mathrm{Ni}}}{M_{\odot}}\right) \times 10^{43} \mathrm{erg} \mathrm{s}^{-1},
$$

where $t_{r}$ is the rise time of the bolometric light curve, and $M_{\mathrm{Ni}}$ is the ${ }^{56} \mathrm{Ni}$ mass (in units of solar masses, $M_{\odot}$ ). With the photometric data in the $R$ band and our earliest unfiltered data from the database of the KAIT survey, we estimate the rise time to the $B$ maximum as $18.4 \pm 0.5$ days. Inserting this value and the maximum bolometric luminosity into the above equation, we derive a nickel mass of $0.78 \pm 0.10 M_{\odot}$ for SN $2005 \mathrm{cf}$. This is within the reasonable range of ${ }^{56} \mathrm{Ni}$ masses for a normal $\mathrm{SN}$ Ia. The quoted error bar includes uncertainties in the rise time and in the peak luminosity. ${ }^{24}$

The lower bolometric luminosity and smaller nickel mass obtained by $\mathrm{P} 07$ is primarily due to differences in the photometry. P07 concluded from their photometry that the host-galaxy extinction was negligible for SN 2005cf, which might not be true

\footnotetext{
24 Assuming an $R_{V}=2.1$, however, the nickel mass produced in the explosion of SN 2005cf would be about $0.70 M_{\odot}$.
}

Table 13

Relevant Parameters for SN 2005cf and its Host

\begin{tabular}{|c|c|c|}
\hline Parameter & Value & Source \\
\hline & Photometric Parameters & \\
\hline Discovery date & 28.26 May 2005 & 1 \\
\hline Epoch of $B$ maximum & $2453533.66 \pm 0.28$ & 2 \\
\hline$B_{\max }$ & $13.63 \pm 0.02 \mathrm{mag}$ & 2 \\
\hline$B_{\max }-V_{\max }$ & $0.07 \pm 0.03 \mathrm{mag}$ & 2 \\
\hline$E(B-V)_{\text {host }}$ & $0.10 \pm 0.03 \mathrm{mag}$ & 2 \\
\hline$\Delta m_{15}($ true $)$ & $1.07 \pm 0.03 \mathrm{mag}$ & 2 \\
\hline$\Delta C_{12}$ (true) & $0.27 \pm 0.03$ & 2 \\
\hline Late-time $B$ decline rate & $1.62 \pm 0.05 \mathrm{mag}(100 \text { days })^{-1}$ & 2 \\
\hline $\mathrm{L}_{\mathrm{bol}}^{\max }$ & $(1.56 \pm 0.20) \times 10^{43} \mathrm{erg} \mathrm{s}^{-1}$ & 2 \\
\hline$t_{r}$ & $18.4 \pm 0.5$ days & 2 \\
\hline \multirow[t]{2}{*}{${ }^{56} \mathrm{Ni}$} & $0.78 \pm 0.10 M_{\odot}$ & 2 \\
\hline & Spectroscopic Parameters & \\
\hline$v_{\max }(\mathrm{Si}$ II $\lambda 6355)$ & $\sim 10,110 \mathrm{~km} \mathrm{~s}^{-1}$ & 2 \\
\hline$v_{\max }(\mathrm{S}$ II $\lambda 5460)$ & $\sim 9780 \mathrm{~km} \mathrm{~s}^{-1}$ & 2 \\
\hline$\dot{v}(\mathrm{Si}$ II $\lambda 6355)$ & $38 \pm 5 \mathrm{~km} \mathrm{~s}^{-1}$ & 2 \\
\hline \multirow[t]{2}{*}{$R(\mathrm{Si} \text { II })_{\max }$} & $0.28 \pm 0.04$ & 2 \\
\hline & Parameters for MCG-01-39-003 & \\
\hline Galaxy type & S0 pec & 3 \\
\hline$E(B-V)_{\mathrm{Gal}}$ & 0.097 & 3 \\
\hline$(m-M)$ & $32.31 \pm 0.11$ & 2 \\
\hline$V_{\text {hel }}$ & $1937 \mathrm{~km} \mathrm{~s}^{-1}$ & 3 \\
\hline
\end{tabular}

Note. Uncertainties are $1 \sigma$.

Reference. (1) Pugh \& Li 2005; (2) this paper; (3) NASA Extragalactic Database.

according to our analysis. Table 13 lists all of the important parameters for SN 2005cf and its host galaxy, as we derived in the previous sections.

\section{DISCUSSION AND CONCLUSIONS}

In this paper, we present extensive optical, UV, and NIR photometry as well as optical spectroscopy of SN 2005cf. In particular, the photometric observations in the optical bands were extremely well-sampled with numerous telescopes. To minimize systematic deviations from the standard system, we carefully computed the $S$-corrections and applied them to SN $2005 \mathrm{cf}$. Photometry without such corrections may potentially lead to a noticeably inconsistent measurement of the reddening, which makes precise extinction corrections difficult.

The Swift UVOT optical photometry is found to be consistent with the ground-based observations to within \pm 0.03 mag, after applying the $S$-corrections. The Swift UVOT UV uvm2 and uvw2 photometry is relatively close to that of HST ACS F220W and F250W, respectively, with a small shift of up to $0.1-0.2$ mag.

Our observations show that SN 2005cf is a normal SN Ia with a typical luminosity decline rate $\Delta m_{15}$ (true) $=1.07 \pm 0.03$ mag. Based on the $V-J H K$ colors and the refurbished Color$\Delta m_{15}$ relations, we estimate the host-galaxy reddening to be $E(B-V)_{\text {host }}=0.10 \pm 0.03 \mathrm{mag}$, which is small but nonnegligible. The NIR light curves closely resemble those of normal $\mathrm{SNe} \mathrm{Ia}$, with a prominent secondary maximum. One distinguishing feature is the UV luminosity in the 2000-2500 $\AA$ range which appears much fainter and peaks later than that of the neighboring bands. This is likely caused by intrinsic spectral features in the UV, an explanation favored by the evolution of the SED.

The comprehensive data, from UV to NIR bands, allow us to establish the uvoir bolometric light curve of SN 2005cf in the 1600-24000 A range. The maximum bolometric luminosity is 
found to be $(1.56 \pm 0.20) \times 10^{43} \mathrm{erg} \mathrm{s}^{-1}$, corresponding to a synthesized nickel mass of $0.78 \pm 0.10 M_{\odot}$. The bolometric luminosity is dominated by the optical emission; the UV contribution is found to be a few percent of the optical, and the peak value $(\sim 10 \%)$ occurs at around maximum light. The NIR flux contribution shows more complicated temporal evolution: the ratio decreases from the beginning of the explosion and reaches a minimum of $\sim 5 \%$ at $t \approx+5$ days; it then rises up to a peak value of $\sim 20 \%$ at $t \approx 35$ days, and finally declines in a linear fashion.

In general, the optical spectra of SN $2005 \mathrm{cf}$ are similar to those of normal SNe Ia. Strong HV features are distinctly present in the $\mathrm{Ca}$ II NIR triplet and the Ca II H\&K lines; these are detached from the photosphere at a velocity $\sim 20,000-25,000$ $\mathrm{km} \mathrm{s}^{-1}$. An HV Ca II feature is commonly seen in other SNe Ia, while the flat-bottomed shape of the Si II $\lambda 6355$ line is relatively rare perhaps due to the paucity of the very early spectra. The excellent temporal coverage of the spectra of SN 2005cf reveals that either the flat-bottomed feature or the triangular-shaped feature associated with $\mathrm{Si}$ II $\lambda 6355$ in some SNe Ia might be due to contamination by another $\mathrm{HV}$ absorption component, such as a pure Si II shell.

Although $\mathrm{HV}$ absorptions of $\mathrm{Ca}$ II and $\mathrm{Si}$ II are observed in many SNe Ia, they diverge in strength and duration. For instance, the HV features are very strong in SN 2005cf and SN 2001el, remaining until a few days after $B$ maximum, whereas they are relatively weak in SN 2003du and SN 2005cg and become marginally detectable around $B$ maximum. Given a common origin of the HV absorption from the aspherical ejecta (e.g., a torus or clumps), the diversity in strength of the HV features could be interpreted as a line-of-sight effect (see also Tanaka et al. 2006). Aspherical structure of the ejecta is favored by the evidence that the degree of polarization for the line features in SN 2001el is much higher than that for the continuum (Wang et al. 2003b; Kasen et al. 2003; see also Chornock \& Filippenko 2008 for SN 2004S). It is thus interesting to examine whether the strength of the HV line features correlates, at least for a subset of $\mathrm{SNe}$ Ia, with that of the line polarization. Unfortunately, similar high-quality polarimetric measurements at early phases are sparse for SNe Ia (but see Wang et al. 2007). Obviously, polarization spectra obtained from the very beginning would allow us to penetrate the layered geometrical structure of the SN ejecta as well as the immediate environment surrounding the explosion site.

The well observed, multiwavelength data presented in this paper make SN 2005cf a rare "golden standard" example of normal SNe Ia. It could be used as a testbed either for theoretical models of SNe Ia, or for studies of the systematic errors in SN Ia cosmology.

We thank Ryan Chornock and Stephane Blondin for useful discussions. Some of the data presented herein were obtained at the W. M. Keck Observatory, which is operated as a scientific partnership among the California Institute of Technology, the University of California, and the National Aeronautics and Space Administration (NASA); the observatory was made possible by the generous financial support of the W. M. Keck Foundation. We thank the Lick Observatory, Palomar Observatory, NAOC, and CTIO staffs for their assistance with the observations. The research of A.V.F.'s supernova group at UC Berkeley is supported by NSF grant AST-0607485, the TABASGO Foundation, Gary and Cynthia Bengier, the Richard and Rhoda Goldman Fund, US Department of Energy SciDAC grant
DE-FC02-06ER41453, and US Department of Energy grant DE-FG02-08ER41563. Additional, crucial support was provided by NASA grant GO-10182 from the Space Telescope Science Institute (STScI), which is operated by AURA, Inc., under NASA contract NAS5-26555. We are also grateful to the National Natural Science Foundation of China (NSFC grant 10673007), the 973 Key Program of China (2009CB824800), and the Basic Research Funding at Tsinghua University (JCqn2005036). Supernova research at Harvard University is supported by NSF grant AST-0606772. M.M. is supported by a fellowship from the Miller Institute for Basic Research in Science at UC Berkeley. The work of A.G. is supported by grants from the Israeli Science Foundation, the EU Marie Curie IRG program, the Benoziyo Center for Astrophysics, a research grant from the Peter and Patricia Gruber Awards, and the William Z. and Eda Bess Novick New Scientists Fund at the Weizmann Institute. The KAIT and its ongoing operation were made possible by donations from Sun Microsystems, Inc., the Hewlett-Packard Company, AutoScope Corporation, Lick Observatory, the NSF, the University of California, the Sylvia \& Jim Katzman Foundation, and the TABASGO Foundation. The PAIRITEL project is operated by the Smithsonia Astrophysical Observatory (SAO) and was made possible by a grant from the Harvard University Milton Fund, a camera loan from the University of Virginia, and continued support of the SAO and UC Berkeley. PAIRITEL is further supported by the NASA/Swift Guest Investigator grant NNG06GH50G. The CTIO $1.3 \mathrm{~m}$ telescope is operated by the Smart and Moderate Aperture Research Telescope System (SMARTS) Consortium; we are particularly grateful for the scheduling flexibility of SMARTS. The Liverpool Telescope is operated on the island of La Palma by Liverpool John Moores University in the Spanish Observatorio del Roque de los Muchachos of the Instituto de Astrofisica de Canarias with financial support from the UK Science and Technology Facilities Council. We made use of the NASA/IPAC Extragalactic Database (NED), which is operated by the Jet Propulsion Laboratory, California Institute of Technology, under contract with NASA.

\section{APPENDIX}

\section{THE $S$ - AND $K$-CORRECTIONS}

The photometric transformation for SNe Ia from one filter system to another is always tricky and requires many response parameters characterizing the instruments because of the peculiar SED of SNe Ia. According to the description by Stritzinger et al. (2002), the instrumental response $S(\lambda)$ can be simply defined as

$$
S(\lambda)=F(\lambda) \times A(\lambda) \times Q E(\lambda),
$$

Table A1

Wavelength Shifts to Instrumental Response Curves ${ }^{\mathrm{a}}$

\begin{tabular}{lccccc}
\hline \hline Telescope & $U$ & $B$ & $V$ & $R$ & $I$ \\
\hline KAIT $0.8 \mathrm{~m}$ & 29 blue & 20 blue & 9 red & 38 blue & 29 blue \\
CfA $1.2 \mathrm{~m}$ & 29 blue & 58 red & 9 red & 0 & 0 \\
CTIO $1.3 \mathrm{~m}$ & $\ldots$ & 70 blue & 58 blue & 128 blue & 377 blue \\
CTIO $0.9 \mathrm{~m}$ & 6 blue & 0 & 0 & 46 blue & 41 red \\
Palomar $1.5 \mathrm{~m}$ & $\ldots$ & 0 & $17 \mathrm{red}$ & $15 \mathrm{red}$ & $107 \mathrm{blue}$ \\
Lick $1.0 \mathrm{~m}$ & 38 blue & $38 \mathrm{red}$ & $46 \mathrm{red}$ & $15 \mathrm{blue}$ & 58 red \\
Liverpool $2.0 \mathrm{~m}$ & $\ldots$ & 35 blue & 9 red & 0 & 29 red \\
\hline
\end{tabular}

Note.

a All values are measured in Angstrom units. 
Table A2

$K$ - and $S$-Corrections (mag) Added to the $U B V R I$ Magnitudes of SN 2005cf

\begin{tabular}{|c|c|c|c|c|c|c|c|c|c|c|c|c|c|}
\hline UT Date & JD-2,450,000 & Phase $^{\mathrm{a}}$ & $S c_{U}$ & $S c_{B}$ & $S c_{V}$ & $S c_{R}$ & $S c_{I}$ & $K_{U}$ & $K_{B}$ & $K_{V}$ & $K_{R}$ & $K_{I}$ & Inst. $^{\text {b }}$ \\
\hline 2005 May 31 & 3521.75 & -11.91 & 0.038 & -0.042 & -0.012 & -0.022 & 0.015 & -0.087 & 0.004 & 0.014 & 0.016 & 0.017 & 2 \\
\hline 2005 May 31 & 3521.77 & -11.89 & 0.075 & -0.036 & 0.009 & 0 & -0.020 & -0.087 & 0.004 & 0.014 & 0.016 & 0.017 & 1 \\
\hline 2005 Jun 1 & 3522.74 & -10.92 & 0.032 & -0.043 & -0.014 & -0.025 & 0.018 & -0.073 & 0.006 & 0.013 & 0.016 & 0.018 & 2 \\
\hline 2005 Jun 1 & 3522.87 & -10.79 & 0.071 & -0.034 & 0.005 & 0.001 & -0.015 & -0.071 & 0.007 & 0.013 & 0.016 & 0.019 & 1 \\
\hline 2005 Jun 1 & 3523.15 & -10.51 & $\cdots$ & $\cdots$ & $\cdots$ & $\ldots$ & $\ldots$ & -0.069 & 0.007 & 0.013 & 0.016 & 0.019 & 3 \\
\hline 2005 Jun 2 & 3523.77 & -9.89 & 0.021 & -0.040 & -0.015 & -0.027 & 0.017 & -0.060 & 0.008 & 0.013 & 0.016 & 0.019 & 2 \\
\hline 2005 Jun 2 & 3523.87 & -9.79 & 0.063 & -0.033 & 0.003 & 0.002 & -0.011 & -0.059 & 0.009 & 0.013 & 0.016 & 0.019 & 1 \\
\hline 2005 Jun 2 & 3524.13 & -9.53 & $\cdots$ & $\cdots$ & $\cdots$ & $\cdots$ & $\cdots$ & -0.057 & 0.009 & 0.013 & 0.016 & 0.019 & 3 \\
\hline 2005 Jun 3 & 3524.63 & -9.03 & $\ldots$ & -0.033 & -0.028 & 0.025 & 0.037 & $\ldots$ & 0.010 & 0.013 & 0.016 & 0.020 & 4 \\
\hline 2005 Jun 3 & 3524.68 & -8.98 & $\cdots$ & -0.037 & -0.015 & -0.029 & 0.017 & $\cdots$ & 0.010 & 0.013 & 0.016 & 0.020 & 2 \\
\hline 2005 Jun 3 & 3524.79 & -8.87 & $\ldots$ & -0.034 & 0.002 & 0.002 & 0.004 & $\ldots$ & 0.010 & 0.013 & 0.016 & 0.020 & 5 \\
\hline 2005 Jun 3 & 3524.85 & -8.81 & 0.047 & -0.031 & 0 & 0.003 & -0.010 & -0.049 & 0.010 & 0.013 & 0.016 & 0.020 & 1 \\
\hline 2005 Jun 3 & 3525.42 & -8.24 & $\ldots$ & -0.024 & -0.013 & 0.026 & 0.027 & $\ldots$ & 0.011 & 0.013 & 0.016 & 0.020 & 6 \\
\hline 2005 Jun 4 & 3525.69 & -7.97 & 0.007 & -0.035 & -0.016 & -0.03 & 0.018 & -0.043 & 0.011 & 0.012 & 0.016 & 0.020 & 2 \\
\hline 2005 Jun 4 & 3525.76 & -7.90 & $\cdots$ & -0.032 & -0.003 & 0.006 & -0.003 & $\ldots$ & 0.011 & 0.012 & 0.016 & 0.020 & 5 \\
\hline 2005 Jun 4 & 3525.87 & -7.79 & $\cdots$ & -0.031 & -0.009 & 0.007 & 0.026 & $\cdots$ & 0.011 & 0.012 & 0.016 & 0.020 & 7 \\
\hline 2005 Jun 5 & 3526.68 & -6.98 & $\cdots$ & -0.032 & -0.017 & -0.027 & 0.020 & $\cdots$ & 0.012 & 0.012 & 0.017 & 0.019 & 2 \\
\hline 2005 Jun 5 & 3526.75 & -6.91 & 0.042 & -0.031 & -0.004 & $\ldots$ & $\ldots$ & -0.036 & 0.012 & 0.012 & $\ldots$ & $\ldots$ & 5 \\
\hline 2005 Jun 5 & 3527.44 & -6.22 & $\ldots$ & -0.023 & -0.015 & 0.032 & 0.010 & $\ldots$ & 0.012 & 0.012 & 0.018 & 0.019 & 6 \\
\hline 2005 Jun 6 & 3527.64 & -6.02 & $\cdots$ & -0.028 & -0.021 & 0.020 & 0.018 & $\cdots$ & 0.012 & 0.012 & 0.018 & 0.019 & 4 \\
\hline 2005 Jun 6 & 3527.69 & -5.97 & 0.003 & -0.031 & -0.018 & -0.026 & 0.012 & -0.032 & 0.012 & 0.012 & 0.018 & 0.019 & 2 \\
\hline 2005 Jun 6 & 3527.85 & -5.81 & 0.018 & -0.028 & -0.004 & 0.007 & -0.002 & -0.031 & 0.012 & 0.012 & 0.018 & 0.018 & 1 \\
\hline 2005 Jun 6 & 3528.43 & -5.23 & $\cdots$ & -0.023 & -0.016 & 0.036 & 0.001 & $\cdots$ & 0.012 & 0.012 & 0.018 & 0.018 & 6 \\
\hline 2005 Jun 7 & 3528.75 & -4.91 & $\ldots$ & -0.029 & -0.008 & 0.012 & 0.013 & $\ldots$ & 0.012 & 0.011 & 0.019 & 0.018 & 7 \\
\hline 2005 Jun 7 & 3528.84 & -4.82 & 0.012 & -0.028 & -0.004 & 0.009 & -0.002 & -0.028 & 0.012 & 0.011 & 0.019 & 0.017 & 1 \\
\hline 2005 Jun 8 & 3529.43 & -4.23 & $\ldots$ & -0.022 & -0.016 & 0.038 & -0.016 & $\ldots$ & 0.012 & 0.011 & 0.019 & 0.017 & 6 \\
\hline 2005 Jun 8 & 3529.71 & -3.95 & 0.022 & -0.017 & 0.007 & 0.003 & -0.025 & -0.026 & 0.012 & 0.011 & 0.020 & 0.016 & 8 \\
\hline 2005 Jun 8 & 3530.42 & -3.24 & $\cdots$ & -0.021 & -0.017 & 0.041 & -0.021 & $\cdots$ & 0.012 & 0.011 & 0.020 & 0.015 & 6 \\
\hline 2005 Jun 8 & 3530.59 & -3.07 & $\ldots$ & -0.028 & -0.020 & 0.017 & -0.012 & $\ldots$ & 0.012 & 0.011 & 0.021 & 0.015 & 4 \\
\hline 2005 Jun 9 & 3530.68 & -2.98 & -0.011 & -0.03 & -0.019 & -0.022 & -0.024 & -0.025 & 0.012 & 0.011 & 0.021 & 0.015 & 2 \\
\hline 2005 Jun 10 & 3531.67 & -1.99 & -0.012 & -0.03 & -0.019 & -0.021 & -0.04 & -0.024 & 0.011 & 0.010 & 0.022 & 0.013 & 2 \\
\hline 2005 Jun 10 & 3531.79 & -1.87 & $\cdots$ & -0.028 & -0.007 & 0.017 & 0 & $\ldots$ & 0.011 & 0.010 & 0.022 & 0.013 & 7 \\
\hline 2005 Jun 10 & 3531.83 & -1.83 & -0.005 & -0.027 & -0.004 & 0.014 & -0.006 & -0.024 & 0.011 & 0.010 & 0.022 & 0.013 & 1 \\
\hline 2005 Jun 10 & 3532.42 & -1.24 & $\cdots$ & -0.020 & -0.017 & 0.045 & -0.042 & $\cdots$ & 0.011 & 0.010 & 0.022 & 0.012 & 6 \\
\hline 2005 Jun 11 & 3532.87 & -0.79 & 0 & -0.027 & -0.004 & 0.016 & -0.007 & -0.025 & 0.011 & 0.010 & 0.023 & 0.011 & 1 \\
\hline 2005 Jun 11 & 3533.42 & -0.24 & $\ldots$ & -0.019 & -0.017 & 0.054 & -0.050 & $\ldots$ & 0.010 & 0.010 & 0.024 & 0.010 & 6 \\
\hline 2005 Jun 12 & 3533.66 & 0.00 & $\cdots$ & -0.028 & -0.018 & 0.011 & -0.044 & $\cdots$ & 0.010 & 0.010 & 0.024 & 0.009 & 4 \\
\hline 2005 Jun 12 & 3533.72 & 0.06 & $\cdots$ & -0.028 & -0.007 & 0.019 & -0.008 & $\ldots$ & 0.010 & 0.010 & 0.024 & 0.009 & 7 \\
\hline 2005 Jun 12 & 3533.84 & 0.18 & 0 & -0.027 & -0.004 & 0.016 & -0.007 & -0.025 & 0.010 & 0.010 & 0.024 & 0.009 & 1 \\
\hline 2005 Jun 13 & 3534.73 & 1.07 & -0.011 & -0.031 & -0.020 & -0.006 & -0.08 & -0.026 & 0.009 & 0.009 & 0.025 & 0.007 & 2 \\
\hline 2005 Jun 13 & 3534.84 & 1.18 & 0.005 & -0.027 & -0.004 & 0.020 & -0.010 & -0.027 & 0.009 & 0.009 & 0.025 & 0.007 & 1 \\
\hline 2005 Jun 13 & 3535.43 & 1.77 & $\cdots$ & -0.018 & -0.017 & 0.067 & -0.078 & $\ldots$ & 0.009 & 0.009 & 0.025 & 0.006 & 6 \\
\hline 2005 Jun 14 & 3535.72 & 2.06 & $\cdots$ & -0.027 & -0.008 & 0.022 & -0.015 & $\cdots$ & 0.009 & 0.009 & 0.026 & 0.005 & 7 \\
\hline 2005 Jun 14 & 3535.74 & 2.08 & -0.009 & -0.032 & -0.020 & 0.005 & -0.090 & -0.028 & 0.009 & 0.009 & 0.026 & 0.005 & 2 \\
\hline 2005 Jun 14 & 3535.83 & 2.17 & 0.015 & -0.027 & -0.004 & 0.024 & $\cdots$ & -0.028 & 0.008 & 0.009 & 0.026 & $\cdots$ & 1 \\
\hline 2005 Jun 14 & 3536.44 & 2.78 & $\cdots$ & -0.018 & -0.017 & 0.07 & -0.089 & $\cdots$ & 0.008 & 0.008 & 0.026 & 0.004 & 6 \\
\hline 2005 Jun 15 & 3536.70 & 3.04 & -0.004 & -0.032 & -0.020 & 0.007 & -0.103 & -0.029 & 0.008 & 0.008 & 0.026 & 0.003 & 2 \\
\hline 2005 Jun 15 & 3536.83 & 3.17 & 0.017 & -0.027 & -0.004 & 0.024 & -0.012 & -0.030 & 0.008 & 0.008 & 0.027 & 0.003 & 1 \\
\hline 2005 Jun 15 & 3537.47 & 3.81 & $\cdots$ & -0.017 & -0.016 & 0.071 & -0.101 & $\cdots$ & 0.007 & 0.008 & 0.027 & 0.002 & 6 \\
\hline 2005 Jun 16 & 3537.82 & 4.16 & $\cdots$ & $\ldots$ & -0.004 & 0.025 & -0.013 & -0.031 & 0.007 & 0.008 & 0.027 & 0.001 & 1 \\
\hline 2005 Jun 17 & 3538.68 & 5.02 & $\ldots$ & -0.025 & -0.017 & 0.019 & -0.085 & $\ldots$ & 0.006 & 0.007 & 0.028 & 0.001 & 4 \\
\hline 2005 Jun 21 & 3542.61 & 8.95 & $\ldots$ & -0.020 & -0.015 & 0.024 & -0.104 & $\ldots$ & 0.002 & 0.005 & 0.028 & -0.007 & 4 \\
\hline 2005 Jun 21 & 3542.72 & 9.06 & 0.035 & -0.015 & -0.004 & 0.021 & -0.016 & -0.040 & 0.002 & 0.005 & 0.028 & -0.007 & 1 \\
\hline 2005 Jun 21 & 3542.76 & 9.10 & $\cdots$ & -0.024 & -0.013 & 0.022 & -0.023 & $\cdots$ & 0.002 & 0.005 & 0.028 & -0.007 & 7 \\
\hline 2005 Jun 21 & 3543.06 & 9.40 & $\cdots$ & $\cdots$ & $\cdots$ & $\cdots$ & $\cdots$ & -0.040 & 0.002 & 0.005 & 0.028 & -0.007 & 3 \\
\hline 2005 Jun 22 & 3543.68 & 10.02 & 0.016 & -0.024 & -0.018 & 0.015 & -0.160 & -0.041 & 0.001 & 0.004 & 0.027 & -0.008 & 2 \\
\hline 2005 Jun 22 & 3543.82 & 10.16 & 0.040 & -0.013 & -0.004 & 0.021 & -0.018 & -0.042 & 0.001 & 0.004 & 0.027 & -0.008 & 1 \\
\hline 2005 Jun 23 & 3544.77 & 11.11 & $\ldots$ & -0.024 & -0.014 & 0.023 & -0.020 & $\ldots$ & 0 & 0.003 & 0.027 & -0.009 & 7 \\
\hline 2005 Jun 23 & 3544.80 & 11.14 & 0.045 & -0.011 & -0.004 & 0.018 & -0.019 & -0.043 & 0 & 0.003 & 0.027 & -0.009 & 1 \\
\hline 2005 Jun 24 & 3545.82 & 12.16 & 0.050 & -0.010 & -0.004 & 0.016 & -0.020 & -0.044 & -0.001 & 0.003 & 0.026 & -0.009 & 1 \\
\hline 2005 Jun 25 & 3546.75 & 13.09 & 0.058 & -0.009 & -0.004 & 0.015 & -0.020 & -0.045 & -0.002 & 0.002 & 0.024 & -0.009 & 1 \\
\hline 2005 Jun 26 & 3547.67 & 14.01 & $\cdots$ & -0.014 & -0.012 & 0.026 & -0.108 & $\cdots$ & -0.003 & 0.001 & 0.023 & -0.009 & 4 \\
\hline 2005 Jun 26 & 3547.82 & 14.16 & 0.062 & -0.009 & -0.004 & 0.015 & -0.019 & -0.046 & -0.003 & 0.001 & 0.023 & -0.009 & 1 \\
\hline 2005 Jun 27 & 3548.66 & 15.00 & 0.029 & -0.023 & -0.016 & 0.015 & -0.194 & -0.046 & -0.004 & 0 & 0.022 & -0.009 & 2 \\
\hline 2005 Jun 27 & 3548.79 & 15.13 & 0.064 & -0.009 & -0.005 & 0.014 & -0.016 & -0.047 & -0.004 & 0 & 0.021 & -0.009 & 1 \\
\hline
\end{tabular}


Table A2

(Continued)

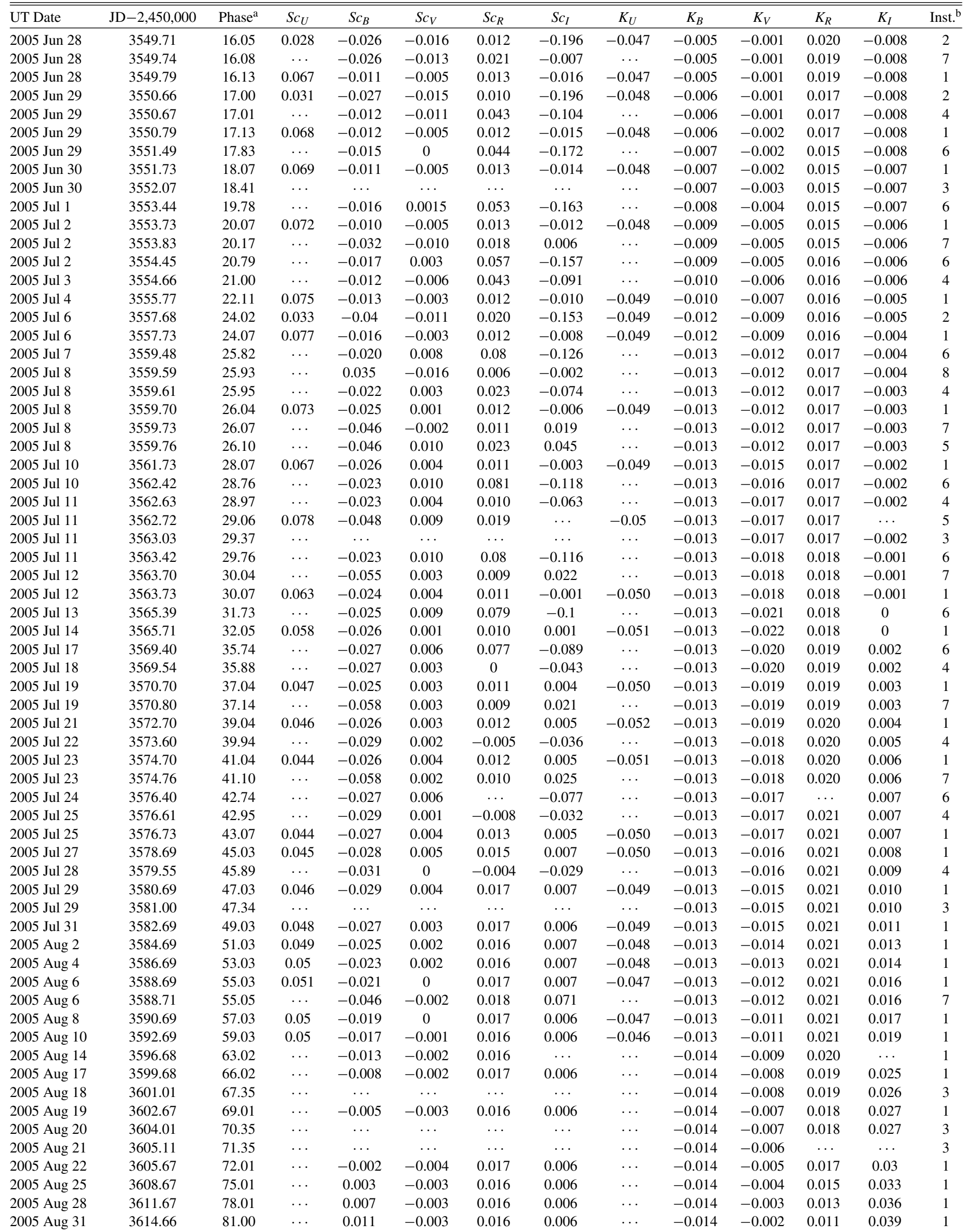


Table A2

(Continued)

\begin{tabular}{lccccccccccccc}
\hline \hline UT Date & JD-2,450,000 & Phase $^{\mathrm{a}}$ & $S c_{U}$ & $S c_{B}$ & $S c_{V}$ & $S c_{R}$ & $S c_{I}$ & $K_{U}$ & $K_{B}$ & $K_{V}$ & $K_{R}$ & $K_{I}$ & Inst. $^{\text {b }}$ \\
\hline 2005 Sept 3 & 3617.66 & 84.00 & $\ldots$ & 0.015 & -0.003 & 0.016 & 0.007 & $\ldots$ & -0.014 & -0.001 & 0.008 & 0.042 & 1 \\
2005 Sept 4 & 3618.65 & 84.99 & $\cdots$ & 0.018 & -0.003 & 0.015 & 0.008 & $\ldots$ & -0.014 & 0 & 0.007 & 0.043 & 1 \\
2005 Sept 7 & 3621.66 & 88.00 & $\cdots$ & 0.022 & -0.001 & 0.012 & 0.009 & $\ldots$ & -0.014 & 0.001 & 0.005 & 0.046 & 1 \\
2005 Sept 10 & 3624.65 & 90.99 & $\cdots$ & 0.026 & 0 & 0.010 & 0.010 & $\ldots$ & -0.014 & 0.002 & 0.002 & 0.049 & 1
\end{tabular}

Notes. ${ }^{a}$ Relative to the epoch of $B$-band maximum $(\mathrm{JD}=2,453,533.66) .{ }^{\mathrm{b}} 1=$ KAIT $0.76 \mathrm{~m} ; 2=$ FLWO $1.2 \mathrm{~m} ; 3=\mathrm{TNT} 0.8 \mathrm{~m} ; 4=\mathrm{CTIO} 1.3 \mathrm{~m} ; 5=\mathrm{Lick} 1.0$ $\mathrm{m} ; 6=$ Liverpool $2.0 \mathrm{~m} ; 7=$ Palomar $1.5 \mathrm{~m} ; 8=$ CTIO $0.9 \mathrm{~m}$.

where $F(\lambda)$ is the filter transmission function, $A(\lambda)$ is the transparency of the Earth's atmosphere, and $Q E(\lambda)$ is the detector quantum efficiency. The atmospheric transmission at the sites where it is not directly available was obtained by modifying the standard atmospheric model (Walker 1987, p. 47) to be consistent with the average broadband absorption coefficients. Here we did not include the mirror reflectivities, dichroic transmission, or dewar window transmissions due to the absence of this information. Various instrumental responses, normalized to the peak transmission, are shown in Figure 2.

To check whether the instrumental response curves match those actually used at the telescopes, we computed the synthetic magnitudes and hence the color terms by convolving the model curves with a large sample of spectrophotometric standard stars from Stritzinger et al. (2005). The resulting synthetic color terms are generally consistent with the values listed in Table 1 of the main text, but small differences are present. The differences are probably due to the mirror reflectivities, dichroic mirror transmission, or other unknown transmissivity of the optical elements. Following the method proposed by Stritzinger et al. (2002), we shifted the wavelength of the model response curves in order to reproduce exactly the measured color terms. The wavelength shifts of different instrumental responses are given in Table A1. They are usually $\lesssim 100 \AA$, except in the $R$ and $I$ filters at the CTIO $1.3 \mathrm{~m}$ telescope where the required shifts are $128 \AA$ and $377 \AA$, respectively. The large shift needed for the CTIO $1.3 \mathrm{~m} R$ filter is not unexpected considering the large departure from the standard band (see Figure 2). While the reason for a large shift in $I$ is unclear, it is perhaps related to other transmissivity (as mentioned above) that was not included in the current instrumental response.

With proper model response curves and better spectral coverage for SN 2005cf, we are able to compute the $S$-corrections using

$$
S c_{\lambda 1}=M_{\lambda 1}-m_{\lambda 1}-C T_{\lambda 1}\left(m_{\lambda 1}-m_{\lambda 2}\right)-Z P_{\lambda 1},
$$

where $M_{\lambda 1}$ is the $\lambda 1$-band SN magnitude synthesized with the Bessell function, and $m_{\lambda 1}$ and $m_{\lambda 2}$ are (respectively) the $\lambda 1$ band and $\lambda 2$-band magnitudes synthesized with the instrumental response function. $C T_{\lambda 1}$ is the color term for the $\lambda 1$ band, and $Z P_{\lambda 1}$ is the zero point which can be determined by performing spectrophotometry of Landolt (1992) standard stars (Stritzinger et al. 2005). In computing the synthetic magnitudes, the photon fluxes (in units of photons $\mathrm{s}^{-1} \mathrm{~cm}^{-2} \AA^{-1}$ ) were used. Since the spectra of SN $2005 \mathrm{cf}$ taken 1-3 months after $B$-band maximum did not have adequate wavelength coverage and were sparsely sampled, we also used the spectra of SN 2003du (Stanishev et al. 2007) to compute the $S$-corrections during that phase.

In order to estimate the corresponding $S$-corrections at any epochs without photometry, a polynomial function was used to fit the data points shown in Figure 3. The resulting $S$-corrections are listed in Table A2 (Columns 4-8).
Owing to a redshift effect on the SED, we further computed the $K$-corrections for SN $2005 \mathrm{cf}$ in the optical bands. The $K$ corrections, based on the response curves of the Bessell filter band and the observed spectra of SNe 2005cf and 2003du, are listed in Table A2 (Columns 9-13). Except in the $U$ band, the $K$-corrections are generally small, $0.02-0.03$ mag around maximum brightness, and they depend on the supernova phase.

\section{REFERENCES}

Anupama, G. C., Sahu, D. K., \& Jose, J. 2005, A\&A, 429, 667

Arnett, W. D. 1982, ApJ, 253, 785

Arnett, W. D., Branch, D., \& Wheeler, J. C. 1985, Nature, 314, 337

Astier, P., et al. 2006, A\&A, 447, 31

Barris, B. J., et al. 2004, ApJ, 602, 571

Benetti, S., et al. 2004, MNRAS, 348, 261

Benetti, S., et al. 2005, ApJ, 623, 1011

Bessell, M. S. 1990, PASP, 102, 1181

Blinnikov, S. I., \& Sorokina, E. I. 2000, A\&A, 356, 30

Blondin, S., et al. 2009, ApJ, 693, 207

Bloom, J. S., Starr, D. L., Blake, C. H., Skrutskie, M. F., \& Falco, E. E. , et al. 2006, in ASP Conf. Ser. 351, Astronomical Data Analysis Software and Systems XV, ed. C. Gabriel (San Francisco, CA: ASP), 751

Branch, D., Baron, E., Thomas, R. C., Kasen, D., Li, W., \& Filippenko, A. V. 2004, PASP, 116, 903

Branch, D., Dang, L. C., \& Baron, E. 2009, PASP, 121, 238

Branch, D., \& Tammann, G. A. 1992, ARA\&A, 30, 359

Branch, D., et al. 2003, AJ, 126, 1489

Branch, D., et al. 2005, PASP, 117, 545

Branch, D., et al. 2006, PASP, 118, 560

Brown, P. J., et al. 2005, ApJ, 635, 1192

Brown, P. J., et al. 2009, AJ, 137, 4517

Candia, P., et al. 2003, PASP, 115, 277

Cardelli, J. A., Clayton, G. C., \& Mathis, J. S. 1989, ApJ, 345, 245

Cenko, S. B., et al. 2006, PASP, 118, 1396

Chornock, R., \& Filippenko, A. V. 2008, AJ, 136, 2227

Conley, A., et al. 2008, ApJ, 681, 482

Cousins, A. W. J. 1981, South African Astron. Obs. Circ., 6, 4

Cutri, R. M., et al. 2003, The IRSA 2MASS All-Sky Point Source Catalog NASA/IPAC Infrared Science Archive (Pasadena, CA: CalTech)

Elias-Rosa, N., et al. 2006, MNRAS, 369, 1880

Ellis, R. S., et al. 2008, ApJ, 674, 51

Faber, S. M., et al. 2003, Proc. SPIE, 4841, 1657

Fabricant, D., Cheimets, P., Caldwell, N., \& Geary, J. 1998, PASP, 110, 79

Filippenko, A. V. 1982, PASP, 94, 715

Filippenko, A. V. 1997, ARA\&A, 35, 309

Filippenko, A. V. 2005a, in White Dwarfs: Cosmological and Galactic Probes, ed. E. M. Sion, S. Vennes, \& H. L. Shipman (Dordrecht: Springer), 97

Filippenko, A. V. 2005b, in ASP. Conf. Ser. 332, The Fate of the Most Massive Stars, ed. R. Humphreys \& K. Stanek (San Francisco, CA: ASP) 33

Filippenko, A. V., Li, W., Treffers, R. R., \& Modjaz, M. 2001, in Small Telescope Astronomy on Global Scales, ed. B. Paczyński, W.-P. Chen, \& C. Lemme (San Francisco, CA: ASP), 121

Filippenko, A. V., et al. 1992a, ApJ, 384, L15

Filippenko, A. V., et al. 1992b, AJ, 104, 1543

Fisher, A. K. 2000, Ph.D. thesis, Univ. of Oklahoma

Fisher, A., Branch, D., Nugent, P., \& Baron, E. 1997, ApJ, 481, 89

Foley, R. J., Filippenko, A. V., \& Jha, S. W. 2008, ApJ, 686, 117

Foley, R. J., Smith, N., Ganeshalingam, M., Li, W. D., Chornock, R., \& Filippenko, A. V. 2007, ApJ, 657, L105

Foley, R. J., et al. 2003, PASP, 115, 1220 
Garavini, G., et al. 2007, A\&A, 471, 527 (G07)

Garnavich, P., et al. 2004, ApJ, 613, 1120

Gehrels, N., et al. 2004, ApJ, 611, 1005

Gerardy, C., et al. 2004, ApJ, 607, 391

Goldhaber, G., et al. 2001, ApJ, 558, 359

Guy, J., Astier, P., Nobili, S., Regnault, N., \& Pain, P. 2005, A\&A, 443, 781

Guy, J., et al. 2007, A\&A, 466, 11

Hamuy, M., et al. 1996a, AJ, 112, 2391

Hamuy, M., et al. 1996b, AJ, 112, 2408

Hamuy, M., et al. 2002, AJ, 124, 417

Hicken, M., Garnavich, P. M., Prieto, J. L., Blondin, S., DePoy, D. L., Kirshner, R. P., \& Parrent, J. 2007, ApJ, 669, 17

Hillebrandt, W., \& Niemeyer, J. C. 2000, ARA\&A, 38, 191

Höflich, P., Wheeler, J. C., \& Thielemann, F.-K. 1998, ApJ, 495, 617

Jha, S., et al. 2006a, AJ, 131, 527

Jha, S., Branch, D., Chornock, R., Foley, R. J., Li, W., Swift, B. J., Casebeer, D., \& Filippenko, A. V. 2006b, AJ, 132, 189

Jha, S., Riess, A. G., \& Kirshner, R. P. 2007, ApJ, 659, 122

Jha, S., et al. 1999, ApJS, 125, 73

Johnson, H. L., Iriarte, B., Mitchell, R. I., \& Wisniewskj, W. Z. 1966, Commun. Lunar Planet. Lab., 4, 99

Kasen, D., et al. 2003, ApJ, 593, 788

Kasen, D., \& Woosley, S. E. 2007, ApJ, 656, 661

Kirshner, R. P., et al. 1993, ApJ, 415, 589

Knop, R. A., et al. 2003, ApJ, 598, 102

Kotak, R., et al. 2005, A\&A, 436, 1021

Krisciunas, K., et al. 2000, ApJ, 539, 658

Krisciunas, K., et al. 2003, AJ, 125, 166

Krisciunas, K., et al. 2004a, AJ, 128, 3034

Krisciunas, K., et al. 2004b, AJ, 127, 1664

Krisciunas, K., et al. 2007, AJ, 133, 58

Landolt, A. V. 1992, AJ, 104, 340

Leibundgut, B., Kirshner, R. P., Filippenko, A. V., Shields, J. C., Foltz, C. B., Phillips, M. M., \& Sonneborn, G. 1991, ApJ, 371, L23

Leibundgut, B., et al. 1993, AJ, 105, 301

Lentz, E. J., Baron, E., Branch, D., Hauschildt, P. H., \& Nugent, P. E. 2000, ApJ, 530, 966

Leonard, D. C. 2007, ApJ, 670, 1275

Leonard, D. C., Li, W., Filippenko, A. V., Foley, R. J., \& Chornock, R. 2005, ApJ, 632,450

Li, W., et al. 2001, PASP, 113, 1178

Li, W., et al. 2003, PASP, 115, 453

Li, W., et al. 2006, PASP, 118, 37

Lira, P. 1995, Masters thesis, Univ. of Chile

Mattila, S., Lundqvist, P., Sollerman, J., Kozma, C., Baron, E., Fransson, C., Leibundgut, B., \& Nomoto, K. 2005, A\&A, 443, 649

Mazzali, P. A., et al. 2001, ApJ, 547, 988

Mazzali, P. A., et al. 2005, ApJ, 623, L37

Miller, J. S., \& Stone, R. P. S. 1993, Lick Obs. Tech. Rep. No. 66

Modjaz, M., Kirshner, R., \& Challis, P. 2005, IAU Circular No. 8534

Nugent, P., et al. 1995, ApJ, 455, L147

Oke, J. B., et al. 1995, PASP, 107, 375

Panagia, N., et al. 2003, in Supernovae and Gamma-Ray Bursters, ed. K. Weiler (Berlin: Springer), 113

Pastorello, A., et al. 2007a, MNRAS, 377, 1531

Pastorello, A., et al. 2007b, MNRAS, 376, 1301, P07

Patat, F., Benetti, S., Cappellaro, E., Danziger, I. J., Della Valle, M., Mazzali, P. A., \& Turatto, M. 1996, MNRAS, 278, 111
Pavlovsky, C. I., et al. 2004, in ACS Instrument Handbook, Version 5.0, (Baltimore, MD: STScI)

Perlmutter, S., et al. 1997, ApJ, 483, 565

Perlmutter, S., et al. 1999, ApJ, 517, 565

Persson, S. E., Murphy, D. C., Krzeminski, W., Roth, M., \& Rieke, M. J. 1998, AJ, 116, 2475

Phillips, M. M. 1993, ApJ, 413, L105

Phillips, M. M., et al. 1992, AJ, 103, 1632

Phillips, M. M., et al. 1999, AJ, 118, 1766 (P99)

Pignata, G., et al. 2004, MNRAS, 355, 178

Pignata, G., et al. 2008, MNRAS, 388, 971

Poole, T. S., et al. 2008, MNRAS, 383, 627

Prieto, J. L., Rest, A., \& Suntzeff, N. B. 2006, ApJ, 647, 501

Pugh, H., \& Li, W. 2005, CBET, 158

Quimby, R., Höflich, P., Kannappan, S. J., Rykoff, E., Rujopakarn, W., Akerlof, C. W., Gerardy, C. L., \& Wheeler, J. C. 2006, ApJ, 636, 400

Riess, A. G., Press, W. H., \& Kirshner, R. P. 1996, ApJ, 473, 588

Riess, A. G., et al. 1998, AJ, 116, 1009

Riess, A. G., et al. 1999, AJ, 117, 707

Riess, A. G., et al. 2004, ApJ, 607, 665

Riess, A. G., et al. 2005, ApJ, 627, 579

Riess, A. G., et al. 2007, ApJ, 659, 98

Roming, P. W. A., et al. 2005, Space Sci. Rev., 120, 95

Röpke, F. K., Gieseler, M., Reinecke, M., Travaglio, C., \& Hillebrandt, W. 2006, A\&A, 453, 203

Salvo, M. E., Cappellaro, E., Mazzali, P. A., Benetti, S., Danziger, I. J., Patat, F., \& Turatto, M. 2001, MNRAS, 321, 254

Sauer, D., et al. 2008, MNRAS, 391, 1605

Schlegel, D. J., Finkbeiner, D. P., \& Davis, M. 1998, ApJ, 500, 525

Sirianni, M., et al. 2005, PASP, 117, 1049

Stanishev, V., et al. 2007, A\&A, 469, 645

Stetson, P. B. 1987, PASP, 99, 191

Stritzinger, M., \& Leibundgut, B. 2005, A\&A, 431, 423

Stritzinger, M., et al. 2002, AJ, 124, 2100

Stritzinger, M., et al. 2005, PASP, 117, 810

Suntzeff, N. B. 1996, in Supernovae and Supernova Remnants, ed. R. McCray \& Z. R. Wang (Cambridge: Cambridge Univ. Press), 41

Tanaka, M., Mazzali, P. A., Maeda, K., \& Nomoto, K. 2006, ApJ, 645, 470

Tanaka, M., et al. 2008, ApJ, 677, 448

Thomas, R. C., Branch, D., Baron, E., Nomoto, K., Li, W., \& Filippenko, A. V. 2004, ApJ, 601, 1019

Tonry, J. L., et al. 2003, ApJ, 594,

Turatto, M., Benetti, S., \& Cappellaro, E. 2003, in From Twilight to Highlight: The Physics of Supernovae, ed. W. Hillebrandt \& B. Leibundgut (Berlin: Springer), 200

Walker, G. 1987, Astronomical Observations (Cambridge: Cambridge Univ Press)

Wang, L., Baade, D., \& Patat, F. 2007, Science, 315, 212

Wang, L., Goldhaber, G., Aldering, G., \& Perlmutter, S. 2003a, ApJ, 590, 944

Wang, L., et al. 2003b, ApJ, 591, 1110

Wang, X., et al. 2008a, ApJ, 675, 626

Wang, X., Li, W., Filippenko, A. V., Foley, R. J., Smith, N., \& Wang, L. 2008b, ApJ, 677, 1060

Wang, X., Wang, L., Zhou, X., Lou, Y., \& Li, Z. 2005, ApJ, 620, L87

Wang, X., et al. 2006, ApJ, 645, 488

Wood-Vasey, W. M., et al. 2007, ApJ, 666, 694

Wood-Vasey, W. M., et al. 2008, ApJ, 689, 377 